University of Louisville

ThinkIR: The University of Louisville's Institutional Repository

\title{
3-D lung deformation and function from respiratory-gated 4-D x- ray CT images : application to radiation treatment planning.
}

Mohammadreza Negahdar 1979-

University of Louisville

Follow this and additional works at: https://ir.library.louisville.edu/etd

\section{Recommended Citation}

Negahdar, Mohammadreza 1979-, "3-D lung deformation and function from respiratory-gated 4-D x-ray CT images : application to radiation treatment planning." (2012). Electronic Theses and Dissertations. Paper 1048.

https://doi.org/10.18297/etd/1048

This Doctoral Dissertation is brought to you for free and open access by ThinkIR: The University of Louisville's Institutional Repository. It has been accepted for inclusion in Electronic Theses and Dissertations by an authorized administrator of ThinkIR: The University of Louisville's Institutional Repository. This title appears here courtesy of the author, who has retained all other copyrights. For more information, please contact thinkir@louisville.edu. 


\title{
3-D LUNG DEFORMATION AND FUNCTION FROM RESPIRATORY-GATED 4-D X-RAY CT IMAGES: APPLICATION TO RADIATION TREATMENT PLANNING
}

\author{
By \\ Mohammadreza Negahdar \\ M.Sc., Tehran University of Medical Sciences, Iran, 2006
}

\begin{abstract}
A Dissertation
Submitted to the Faculty of the

Speed School of Engineering of the University of Louisville in Partial Fulfillment of Requirements

for the Degree of

Doctor of Philosophy
Department of Electrical and Computer Engineering
University of Louisville
Louisville, Kentucky

August 2012 
3-D LUNG DEFORMATION AND FUNCTION FROM RESPIRATORY-GATED 4-D X-RAY IMAGES: APPLICATION TO RADIATION TREATMENT PLANNING

By

Mohammadreza Negahdar

M.Sc., Tehran University of Medical Sciences, Iran, 2006

A Dissertation Approved on

June 21, 2012

by the following Dissertation Committee:

Amir Amini, Dissertation Director 


\section{ACKNOWLEDGEMENTS}

First and foremost, the members of my dissertation committee have my profound appreciation for their ideas and suggestions. I would like to express my sincere gratitude to my advisor Dr. Amir A. Amini, whose professionalism, ambition, passion, and guidance I appreciate. While at the Medical Imaging Lab, I enjoyed freedom of exploration and owe my confidence to perform independent research to his encouragement. In addition to providing financial support and suggesting measurement of strain as a marker of regional lung function as a thesis topic, Dr. Amini spent countless hours discussing the technical approach, providing comments and help with the derivations, and helping prepare manuscripts and papers. I also would like to give my sincere thanks to Dr. Shiao Y. Woo for welcoming me to the Brown Cancer Center with open arms for being highly enthusiastic about this study. Many thanks also go to Dr. Neal Dunlap who is a highly energetic collaborator and who designed the protocol for the patient studies. Collaborating with Neal has been fun and enjoyable. I would also like to express my heartfelt thanks to the other members of my committee: Dr. Rafael L. Perez, Dr. Jacek M. Zurada, and Dr. Tamer Inanc, for their precious time and invaluable comments on my dissertation. I would also like to mention my M.S. advisor, Dr. Nassir Navab at the Technical University of Munich, who introduced me to lung imaging and was a perpetual source of inspiration.

I would also like to acknowledge the professionals at the Brown Cancer Center as well as the Department of Radiation Oncology and the Department of Radiology at the University of Louisville; in particular, I benefitted from vivid discussions with Dr. Albert. Zacarias and Dr. A. Cahid Civelek. I would also like to offer my appreciation to Dr. Bronwyn Williams, Mr. Adam Robinson, and Ms. Ashly Bender at the Writing Center of the University of Louisville who compassionately helped me to revise drafts of my dissertation. The Ralph Wright Natatorium of University of Louisville has been the ultimate place for relaxation during all of my graduate studies - swimming is indeed an important part of my life. 
Finally, no words can describe how blessed I feel for the constant love and support afforded to me by my brother, MJ, and my sister, Elaheh. I thank my parents for their love and support, and for instilling within me the need for creativity and for critical thinking. This dissertation is dedicated to them. 


\begin{abstract}
3-D LUNG DEFORMATION AND FUNCTION FROM RESPIRATORY-GATED 4-D X-RAY CT IMAGES: APPLICATION TO RADIATION TREATMENT PLANNING
\end{abstract}

\author{
Mohammadreza Negahdar
}

June 21, 2012

Many lung diseases or injuries can cause biomechanical or material property changes that can alter lung function. While the mechanical changes associated with the change of the material properties originate at a regional level, they remain largely asymptomatic and are invisible to global measures of lung function until they have advanced significantly and have aggregated. In the realm of external beam radiation therapy of patients suffering from lung cancer, determination of patterns of pre- and posttreatment motion, and measures of regional and global lung elasticity and function are clinically relevant. In this dissertation, we demonstrate that 4-D CT derived ventilation images, including mechanical strain, provide an accurate and physiologically relevant assessment of regional pulmonary function which may be incorporated into the treatment planning process.

Our contributions are as follows:

(i) A new volumetric deformable image registration technique based on 3-D optical flow (MOFID) has been designed and implemented which permits the possibility of enforcing physical constraints on the numerical solutions for computing motion field from respiratory-gated 4-D CT thoracic images. The proposed optical flow framework is an accurate motion model for the thoracic CT registration problem. 
(ii) A large displacement landmark-base elastic registration method has been devised for thoracic CT volumetric image sets containing large deformations or changes, as encountered for example in registration of pre-treatment and post-treatment images or multi-modality registration .

(iii) Based on deformation maps from MOFID, a novel framework for regional quantification of mechanical strain as an index of lung functionality has been formulated for measurement of regional pulmonary function.

(iv) In a cohort consisting of seven patients with non-small cell lung cancer, validation of physiologic accuracy of the 4-D CT derived quantitative images including Jacobian metric of ventilation, $V_{J a c}$, and principal strains, $\left(V_{\lambda_{1}}, V_{\lambda_{2}}, V_{\lambda_{3}}\right)$, has been performed through correlation of the derived measures with SPECT ventilation and perfusion scans. The statistical correlations with SPECT have shown that the maximum principal strain pulmonary function map derived from MOFID, outperforms all previously established ventilation metrics from 4D-CT.

It is hypothesized that use of CT-derived ventilation images in the treatment planning process will help predict and prevent pulmonary toxicity due to radiation treatment. It is also hypothesized that measures of regional and global lung elasticity and function obtained during the course of treatment may be used to adapt radiation treatment. Having objective methods with which to assess pre-treatment global and regional lung function and biomechanical properties, the radiation treatment dose can potentially be escalated to improve tumor response and local control. 


\section{TABLE OF CONTENTS}

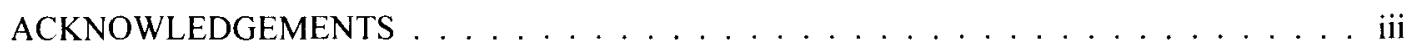

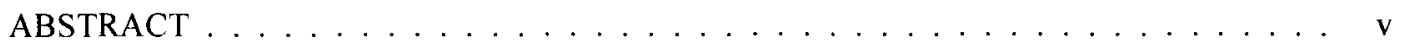

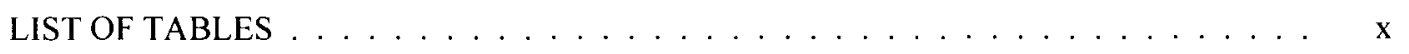

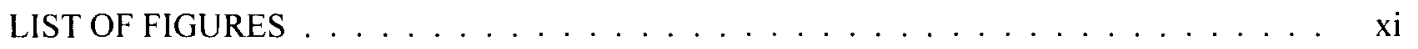

\section{CHAPTER}

1. INTRODUCTION ......................... 1

1.1 Lung Anatomy and Respiratory Physiology . . . . . . . . . . . . . 1

1.2 Lung Cancer $\ldots \ldots \ldots \ldots \ldots \ldots \ldots \ldots$

1.3 Pulmonary CT Imaging . . . . . . . . . . . . . . . 6

$1.3 .1 \quad 4-\mathrm{D}$ CT Lung Imaging $\ldots \ldots \ldots \ldots \ldots$

1.4 Radiotherapy . . . . . . . . . . . . . . . . . . . . 9

1.4.1 Stereotactic Radiation Therapy . . . . . . . . . . . . . 11

1.5 Deformable Image Registration $\ldots \ldots \ldots \ldots \ldots$

1.5.1 Thoracic CT Registration . . . . . . . . . . . . . . 13

1.6 Regional Ventilation $\ldots \ldots \ldots \ldots \ldots \ldots$

1.6.1 Regional Ventilation from Image Registration . . . . . . . . . . . . 16

1.7 Applications and Significance of Proposed Work . . . . . . . . . . 16

1.8 Organization of the Dissertation $\ldots \ldots \ldots \ldots \ldots$ 
2. REVIEW OF LITERATURE ON DEFORMABLE IMAGE REGISTRATION . . . . . 19

2.1 Introduction . . . . . . . . . . . . . . . . . . . . . . . 19

2.2 Deformable Image Registration Methods $\ldots \ldots \ldots \ldots$

2.2 .1 Constant Intensity Methods . . . . . . . . . . . . . . . . . . 22

2.2 .2 Varying Intensity Methods $\ldots \ldots \ldots \ldots . \ldots \ldots$

2.3 Deformable Lung Registration Methods . . . . . . . . . . . . . . . 26

2.4 Performance Evaluation of Lung Registration Methods . . . . . . . . . . . 28

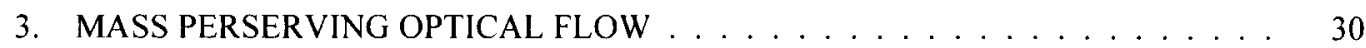

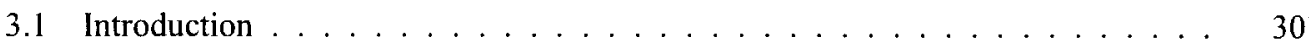

3.2 The Variational Formulation $\ldots \ldots \ldots \ldots \ldots \ldots \ldots \ldots \ldots$

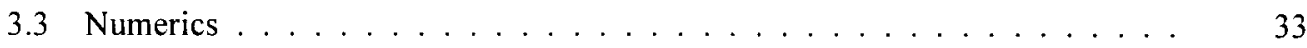

3.4 Experimental Results . . . . . . . . . . . . . . . . . . . . . . . . . . 37

3.4.1 Two Dimensional Experiments . . . . . . . . . . . . . . . . . . 37

3.4.2 Three Dimensional Experiments . . . . . . . . . . . . . 40

4. LANDMARK-BASED ELASTIC REGISTRATION FOR ESTIMATING LARGE DEFORMATIONS . . . . . . . . . . . . . . . . . . 50

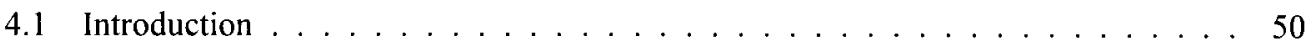

4.2 Path Planning and Landmark Extraction $\ldots \ldots \ldots \ldots \ldots$

4.3 Transformation Estimation . . . . . . . . . . . . . . . . 53

4.4 Experimental Results . . . . . . . . . . . . . . . . . . . . . . 53

5. MEASURES OF REGIONAL MECHANICS FROM DEFORMATION MAPS . . . . . 57

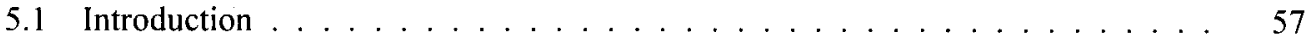

5.2 Regional Measures of Mechanics from Image Registration . . . . . . . . . . 58

5.3 Visualization of 3-D Strain Tensor Field . . . . . . . . . . . . . . 61

5.4 Relationship between Jacobian and Principal Strains . . . . . . . . . . . 62

5.5 Experimental Results . . . . . . . . . . . . . . . . . 63 
6. PHYSIOLOGIC VALIDATION OF MEASURES OF REGIONAL PULMONARY

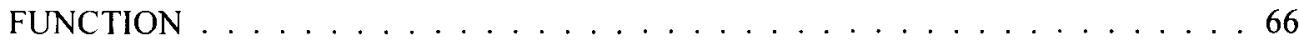

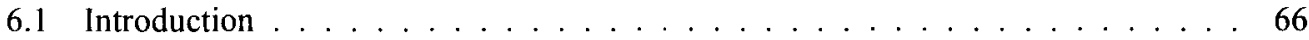

6.2 Patient Studies . . . . . . . . . . . . . . . . . . . . . . 67

6.3 Method ............................. 68

6.3.1 Deformable Image Registration $\ldots \ldots \ldots \ldots 6$

6.3 .2 Lung Parenchyma Segmentation . . . . . . . . . . . . . 69

6.3.3 Measures of Regional Function from Deformation Maps . . . . . . . . . 69

6.3 .4 Co-Registration of CT and SPECT Data . . . . . . . . . . . 69

6.3 .5 Statistical Analysis . . . . . . . . . . . . . . . 70

6.4 Results . . . . . . . . . . . . . . . . . . . . . . . 70

6.5 Discussion . . . . . . . . . . . . . . . . . . 73

7. CONCLUSIONS AND FUTURE DIRECTIONS . . . . . . . . . . . . . . . . 92

7.1 Motivations . . . . . . . . . . . . . . . . . 92

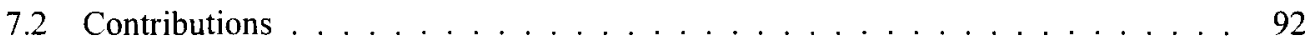

7.3 Future Studies . . . . . . . . . . . . . . . . . . . . . . . . . 94

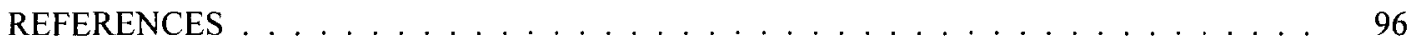

LIST OF ABBREVATIONS . . . . . . . . . . . . . . . . . . . . . . . . . . . 109

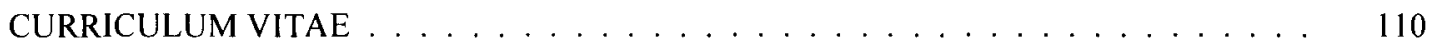




\section{LIST OF TABLES}

3.1 Quantitative evaluation and comparison of the already implemented methods and MOFID . 41

3.2 Spatial accuracy of MOFID for DIR-lab data sets . . . . . . . . . . . . . . . 42

3.3 Spatial accuracy of MOFID in comparison with COF and CCLG . . . . . . . . 47

6.1 The characteristics of patient data included in this study . . . . . . . . . . . 68

6.2 $\rho$ and $P C C$ between SPECT ventilation and 4-D CT derived images for seven subjects . . . 71

6.3 $\rho$ and PCC between SPECT perfusion and 4-D CT derived images for seven subjects. .72 


\section{LIST OF FIGURES}

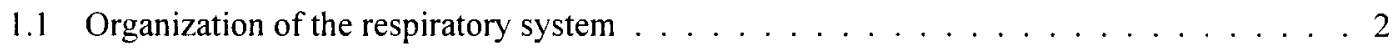

1.2 Definition of lung volumes and capacities $\ldots \ldots \ldots \ldots \ldots \ldots$

1.3 Lung cancer distribution in the United States $\ldots \ldots \ldots \ldots \ldots$

1.4 Tobacco use in the United States, $1900-2006 \ldots \ldots \ldots \ldots \ldots$

1.5 The four generations of $\mathrm{CT} \ldots \ldots \ldots \ldots \ldots$

1.6 Retrospective $4-\mathrm{D}$ CT Lung Imaging $\ldots \ldots \ldots \ldots \ldots$

1.7 Target volumes for radiotherapy planning . . . . . . . . . . . 10

1.8 Image registration as a spatial transformation $\ldots \ldots \ldots \ldots \ldots \ldots \ldots \ldots \ldots$

1.9 The basic components of the image registration framework $\ldots \ldots \ldots \ldots$

1.10 Dynamic ventilation map image . . . . . . . . . . . . 15

3.1 An axial CT slice of lung and the corresponding gradient image $\ldots \ldots \ldots \ldots$

3.2 Similarity calculation between the first warped image and the second image . . . . . 37

3.3 Color wheel for encoding estimated motion vectors $\ldots \ldots \ldots \ldots \ldots$

3.4 Synthetic sponge and Yosemite image sequence motion estimation . . . . . . . . . . . 39

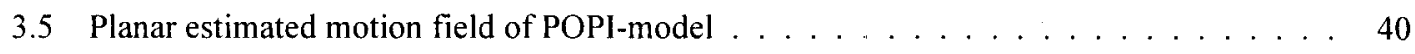

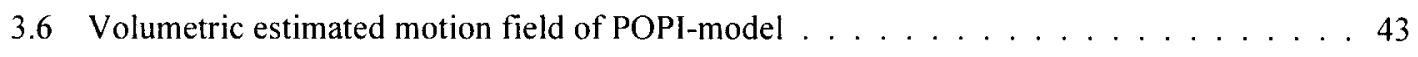

3.7 Distribution of Jacobian between the consecutive phases of POPI-model . . . . . . . . 44

3.8 Phase-step TRE for POPI-model $\ldots \ldots \ldots \ldots \ldots \ldots \ldots \ldots$

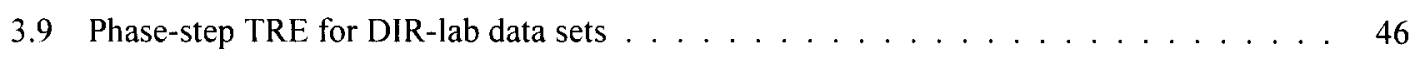

3.10 Sensitivity of TRE to different $\alpha$ and $\gamma$ values $\ldots \ldots \ldots \ldots \ldots$

3.11 Behavior of the calculated energy function in terms of SOR iterations . . . . . . . 49 
4.1 A pair of breath-hold lung images . . . . . . . . . . . . . . . . . . . 51

4.2 Follow-up lung images of TomoTherapy SBRT . . . . . . . . . . . . . . . . . . . 52

4.3 Extracted landmark points with path planning method . . . . . . . . . . . . 53

4.4 Difference images before and after Landmark-based elastic registration . . . . . . . . . . 54

4.5 Histogram of difference images before and after Landmark-based elastic registration . . 55

5.1 Deformation of a continuum body . . . . . . . . . . . . . . 58

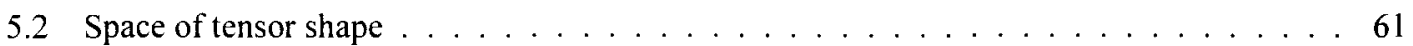

5.3 Estimated motion and strain map of the synthetic sponge image . . . . . . . . . . . 63

5.4 Estimated motion and strain map of the POPI-model . . . . . . . . . . . . . . . 64

5.5 RRMS error of estimated motion and Lagrangian strain in terms of Gaussian noise . . . . . 52

6.1 SPECT ventilation and SPECT perfusion images of all patients at mid-coronal slice . . . . 76

6.2 Volumetric and projected calculated motion field of first patient . . . . . . . . . . . . . 77

6.3 Segmented lung from the 4-D CT images of each patient . . . . . . . . . . . 78

6.4 Calculated Jacobian at anatomical planes . . . . . . . . . . . . . . . . . . . 79

6.5 Calculated principal strain values at anatomical planes . . . . . . . . . . . . . 80

6.6 SPECT ventilation and SPECT perfusion images of first patient at anatomical planes . . . 81

6.7 4-D CT derived quantitative images at mid-planes mapped into SPECT ventilation space . 82

6.8 4-D CT derived quantitative images at mid-planes mapped into SPECT perfusion space . 83

6.9 Distribution of 4-D CT derived quantitative images versus SPECT images in patient one . . 84

6.10 Distribution of 4-D CT derived quantitative images versus SPECT images in patient two . 85

6.11 Distribution of 4-D CT derived quantitative images versus SPECT images in patient three . 86

6.12 Distribution of 4-D CT derived quantitative images versus SPECT images in patient four . 87

6.13 Distribution of 4-D CT derived quantitative images versus SPECT images in patient five . 88

6.14 Distribution of 4-D CT derived quantitative images versus SPECT images in patient six . 89

6.15 Distribution of 4-D CT derived quantitative images versus SPECT images in patient seven . 90

6.16 Comparison of correlation values of $\lambda_{1}$ and Jacobian with SPECT images for all patients . 91 


\section{CHAPTER 1}

\section{INTRODUCTION}

\subsection{Lung Anatomy and Respiratory Physiology}

Lungs are essential organs for respiration, facilitating gas exchange between human body and the atmosphere. They deliver oxygen from the air into the venous blood and extract carbon dioxide from the blood at the same air-blood interface. Moreover, they play other critical roles in physiology, such as rapid modulation of blood $\mathrm{pH}$, thermoregulation, and immunoprotection. Each lung is attached by its root and pulmonary ligament to the heart and trachea but is otherwise free in the thoracic cavity. Lungs are light, soft, spongy, and elastic and because they contain air they float in water. Human lungs consist of the left and right lungs, and the two lungs are located in the chest on either side of the heart. Each lung has an apex, three surfaces (costal, medial, and diaphragmatic), and three borders (anterior, inferior, and posterior). Each lung is surrounded by a completely closed pleural cavity. The pleural folds back into itself and forms a twolayered, membrane structure. The outer pleura (parietal pleura) is attached to the chest wall. The inner pleura (visceral pleura) covers the lungs and divides the two lungs into five lobes. The left lung has two lobes; left upper lobe (LUL) and left lower lobe (LLL), separated by an oblique fissure. The right lung is partitioned into three lobes; right upper lobe (RUL), right middle lobe (RML), and right lower lobe (RLL). The RUL and RML are separated by a horizontal fissure and the RML and RLL are separated by an oblique fissure. A lobar fissure is a thin space (approximately $0.5 \mathrm{~mm}$ depending on volume of pleural fluid) separating the lung lobes. Lung lobes can slide against the chest wall and adjacent lobes during respiration $[36,142]$, and this motion may provide the means to reduce the lung parenchymal distortion and avoid regions of high local stress [70].

Figure 1.1 provides an overview of the respiratory system. As illustrated in Figure 1.1, the trachea branches into two bronchi. Gas is transported between the lungs and the atmosphere through airways, which consist of a series of branching tubes with progressively decreasing dimensions. Within the lung, 


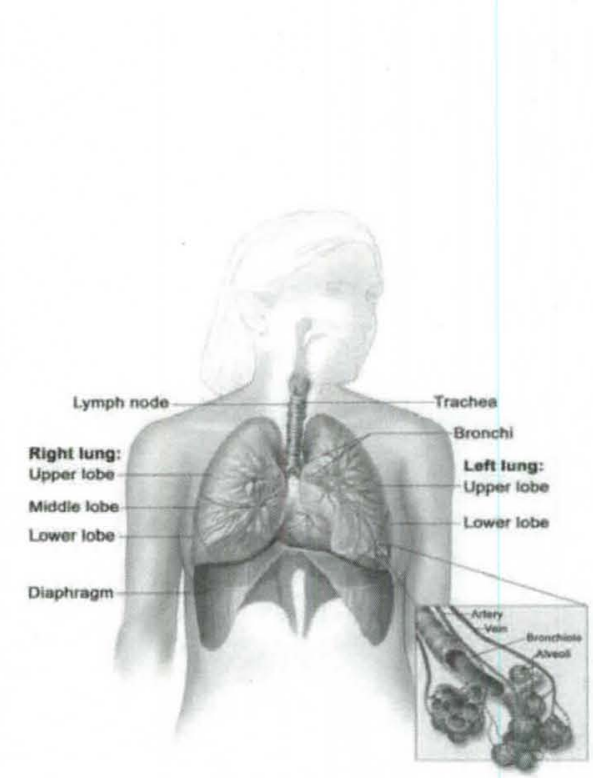

Figure 1.1: Organization of the respiratory system [1].

there are more than 20 generations of airway branching. It is believed that such a bifurcating network connects to about 300 million alveoli, where gas exchange between air and blood takes place. Gas inspiration and expiration are driven by the pressure differences between the atmosphere and chest. The pressure of the intrapleural fluid is called intrapleural pressure $\left(P_{i p}\right)$. The changes of the intrapleural pressure cause the lungs and thoracic wall to move in and out together during normal breathing. The pressure inside the lung is the alveolar pressure $\left(P_{a l v}\right)$. The difference in pressure between the inside and outside of the lung is termed the transpulmonary pressure $\left(P_{t p}\right)$, which is a determinant of lung size, where $P_{t p}=P_{a l v}-P_{i p}$. The transrespiratory system pressure, the difference between the alveolar pressure and the atmospheric pressure $P_{r s}=P_{a l v}-P_{a t m}$, is a determinant for air flow. The intrapleural pressure at rest is a balance between the tendency of the lung to collapse and the tendency of the chest wall to expand. During inspiration, as the diaphragm and the intercostal muscles contract, the thorax expands and the $P_{i p}$ becomes more negative; then the transitionary pressure becomes more positive causing the lungs to expand. The enlargement of the lung causes an increase in the sizes of the alveoli throughout the lung. Therefore, by Boyle's law, the $P_{\text {alv }}$ decreases to become less than $P_{a t m}$ causing a bulk flow of air from the atmosphere through the airways into the alveoli. During expiration, as the diaphragm and inspiratory intercostal muscles stop contracting, the chest wall passively recoils inward to its pre-inspiratory volume, causing the $P_{i p}$ to move back towards its pre-inspiration value. As the lung becomes smaller, air in the alveoli becomes 


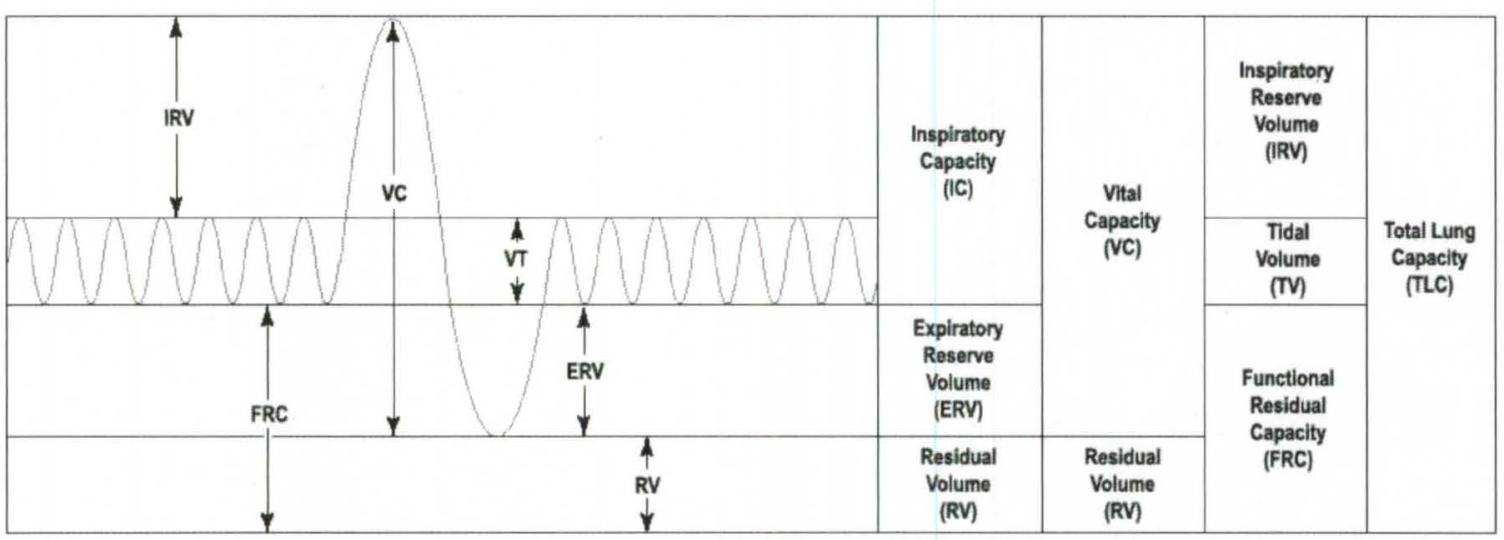

Figure 1.2: Definition of lung volumes and capacities [3].

compressed, so that by Boyle's law, alveolar pressure exceeds atmospheric pressure, therefore air flows from the alveoli through the airways out to the atmosphere.

Lung capacities/volume associated with respiratory physiology are given in Figure 1.2:

Tidal Volume (TV): the air volume breathed in and out during a normal breath. Typical values are around $500 \mathrm{ml}$ for an adult.

Residual Volume $(R V)$ : the amount of air left in the lung after a maximum expiration.

Vital Capacity (VC): the maximum amount of air volume that can be breathed in after a maximum expiration.

Functional residual Capacity $(F R C)$ : the amount of air left in the lungs after a normal expiration.

Total Lung Capacity (TLC): The volume of the lung after maximal inspiration.

Beside the air flow, blood circulates inside the lung parenchyma. Blood is pumped from the right ventricle through the pulmonary arteries and travels through the lungs, where it releases carbon dioxide and picks up oxygen during respiration. The gas exchange occurs between capillaries and alveoli. The oxygenated blood then leaves the lungs through pulmonary veins to the left atrium.

Lung tissue function depends upon the material properties of the lung parenchyma and the relationships between the lungs, diaphragm, and other parts of the respiratory system. Pulmonary diseases/injuries can change the tissue material properties of lung parenchyma. Pulmonary emphysema, a chronic obstructive pulmonary disease (COPD), is characterized by loss of elasticity (increased compliance) of the lung tissue, from destruction of structures supporting the alveoli and destruction of capillaries feeding the alveoli. 


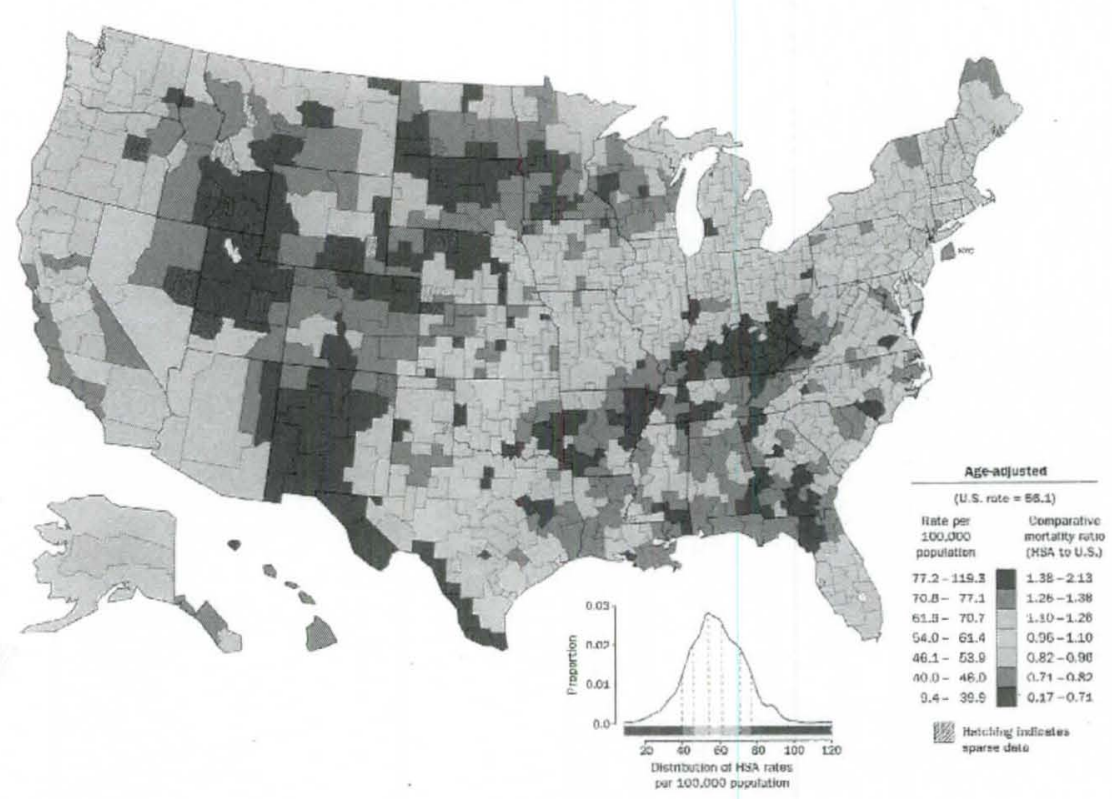

Figure 1.3: Lung cancer distribution in the United States [2].

Idiopathic pulmonary fibrosis (IPF), a classic interstitial lung disease, causes inflammation and fibrosis of tissue in the lungs. Over time, the disease makes the tissue thicker and stiffer (reduced compliance). As the change of structural properties and the disease process per se are associated with the mechanical changes, it would be desirable to have objective methods to determine the regional mechanics which reflect regional pulmonary function.

\subsection{Lung Cancer}

Lung cancer, which is one of the main causes of deaths due to cancer, is a form of very rapid cell growth in the lung tissue caused by changes in tissue form itself. The cells form a mass or tumor that differs from the surrounding tissues from which they arise. Tumors take oxygen, nutrients, and space from healthy cells, and they invade and destroy or reduce the ability of normal tissues to function. Compared with other types of cancer, the number of people diagnosed with lung cancer is rising. The American Cancer Society estimates that in 2012 there will be about 226,160 new diagnoses of lung cancer, accounting for about $14 \%$ of cancer diagnoses, and an estimated 160,340 deaths from lung cancer, accounting for about $28 \%$ of all cancer deaths, which is more than the number of deaths from colorectal, breast, and prostate cancer combined. Figure 1.3 shows the distribution of lung cancer in the United States. 


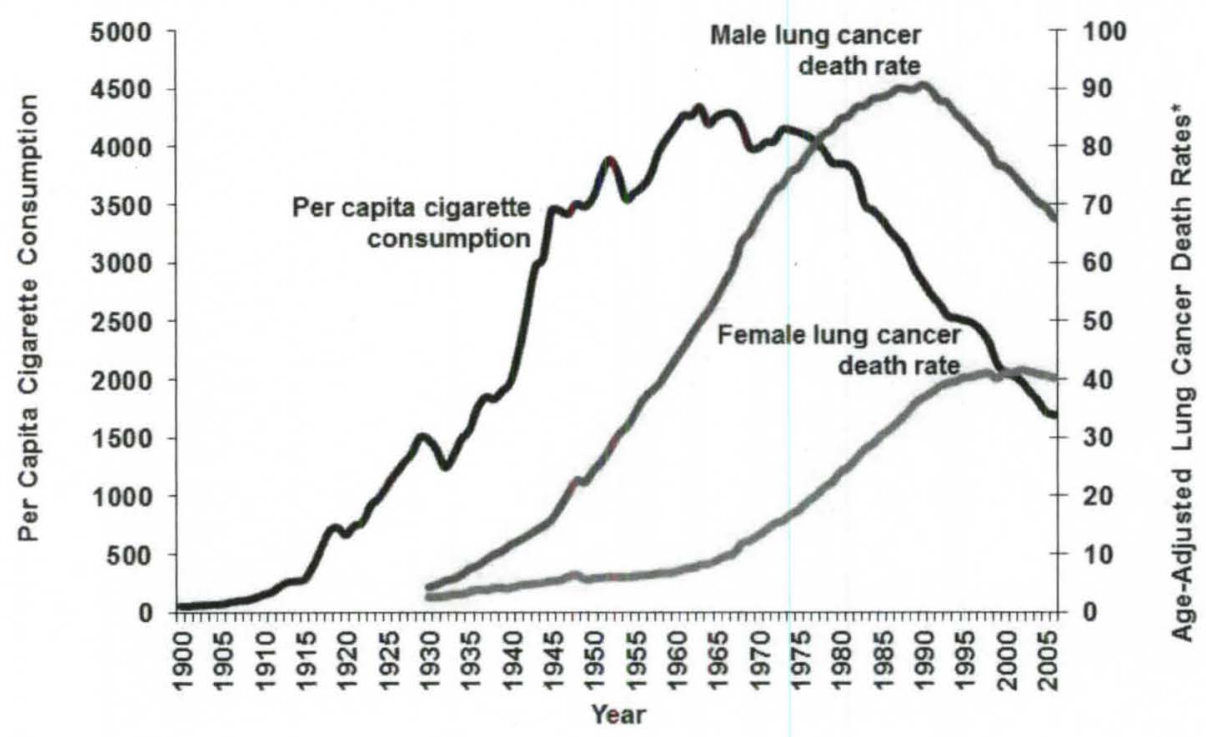

Figure 1.4: Tobacco use in the United States, 1900-2006 (taken from [149]).

Basically lung cancer is divided into two classes:

Primary lung cancer has two types, namely small cell lung cancer (SCLC) and non-small cell lung cancer (NSCLC). Small cell lung cancers account for about 15\% of all lung cancers and have a high degree of malignancy with fast growth and the potential to quickly metastasize. Non-small cell lung cancers account for about $85 \%$ of all lung cancers and result from the growth of single cells and often strike more than one area in the lung, but are not as malignant as SCLC.

Secondary lung cancer arises as a result of the spread of cancer from other organs, most often breast cancer and colon cancer.

Symptoms may include persistent cough, sputum streaked with blood, chest pain, voice change, and recurrent pneumonia or bronchitis. Signs and symptoms of lung cancer will only occur with abnormal and severe cell growth, sometimes many years since the onset of its development. There is even the possibility that the cancer does not result in any specific signs and symptoms, and is only visible in the chest X-ray.

The biggest cause of lung cancer is smoking. Figure 1.4 demonstrates that lung cancer deaths in the United States have a direct relation and in fact have followed the same trend as per capita tobacco consumption between 1930-2005. Exposure to radon gas released from soil and building materials is estimated to be the second leading cause of lung cancer. Other causes are air contamination by asbestos, air pollution by combustion, or fumes including second hand cigarette smoke. There are some diseases that 
1. Generation

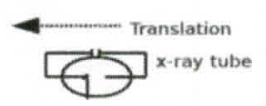

।

।
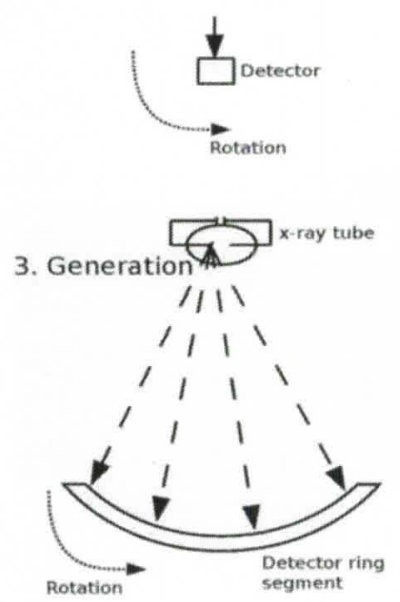

2. Generation

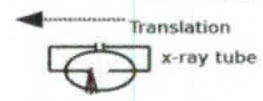

111

111
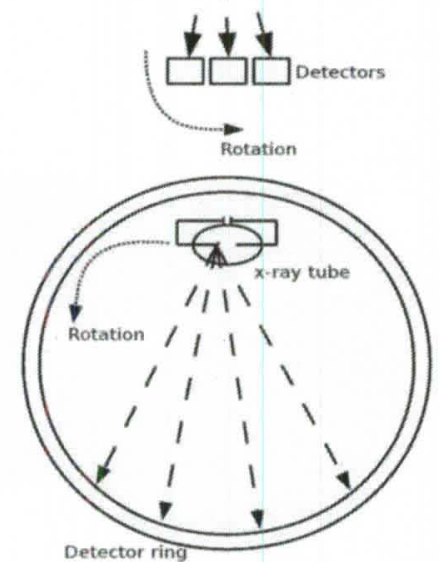

4. Generation

Figure 1.5: The four generations of CT [13].

increase the risk of lung cancer, namely tuberculosis and pneumonia. Both of these diseases can cause injury to the lung tissue organ causing abnormal cell growth within the chest cavity.

Treatment of patients with lung cancer depends on the staging, and can include surgery, chemotherapy, and radiation treatment, or a combination thereof.

\subsection{Pulmonary CT Imaging}

Computed tomography (CT) theory, techniques, and applications have seen continuous development over the years, matching advancement in new acquisition hardware and techniques. The advances in X-ray $\mathrm{CT}$ such as transition from fan-beam to cone-beam geometry, from single-row detector to multiple-row detector arrays, and from conventional to spiral CT now permit a larger scanning range in shorter time and with a higher image resolution $[67,176]$. A schematic showing the development of four generations of CT is shown in Figure 1.5. First generation consisted of a single detector and a sharply collimated X-ray beam. The attenuation profile was recorded during a translation of both the source and detector, which was followed by a rotation of both the detector and the X-ray tube to generate the projection profile for a different angle. Second generation scanners acquired the data in the same manner, but utilized several 
detector elements and an X-ray fan beam with less collimation. A separate translational movement was still part of the acquisition process. In third generation scanners, only a rotation of the curved detector row together with the X-ray tube is carried out. A stationary detector ring with rotating X-ray tube was the fourth generation systems [13]. Helical CT was developed to cover a larger volume of the body in a short time. The data are acquired as the table position is moved continuously in the scanner. Simultaneous motion of the patient bed and rotation of the X-ray source and detectors results in a spiral trajectory of the X-ray transmitted through patient. To date, seven generations of X-ray CT systems have been developed, with the latest generation being cone-beam CT systems having many detector rows (256 rows are now available) and capable of performing helical scans.

CT imaging has been used to study lung since 1980s via the CT-based scanner developed at Mayo Clinic (Rochester, MN), known as the dynamic spatial reconstructor $[65,140,141]$. Because early scanners required up to five seconds for acquiring and reconstruction of a single slice of the lung, CT imaging was primarily used to image static organs. With the emergence of multidetector-row CT (MDCT), it then became possible to image both static and dynamic organs via use of an X-ray imaging modality [64], as had been possible with the use of Ultrasound at that time. MDCT scanners can use multiple detector rows to collect more slices per rotation, thus allowing for increased z-coverage and fast acquisition speed. Current clinical MDCT provides the ability of acquiring up to 256 thin sections with scanner rotation speeds on the order of 0.33 second/revolution for single source $X$-ray tube systems. Operated in a spiral mode, these scanners can acquire images of the lung in a breath hold as short as 5 to 10 seconds. It is possible to achieve near isotropic voxels on the order of $0.5 \mathrm{~mm}$ and image the whole lung in a single breath hold. Highresolution CT imaging is particularly useful for close examination of lung parenchyma and is used to assess a variety of lung pathologies, including interstitial diseases that manifest with different characteristic patterns [170]. Functional information can also be measured with the aid of contrast imaging or postprocessing techniques. For example, regional ventilation can be quantitatively assessed with Xenonenhance CT (Xe-CT) $[23,86,99]$ and regional perfusion can be estimated with bolus contrast injection. The main disadvantage of CT imaging is that it exposes patients to harmful ionizing radiation [102]. An area of increasing research activity involves techniques for dose reduction with simultaneous preservation 
of image quality - for example by using novel X-ray source geometries or use of iterative reconstruction techniques in place of standard filtered back-projection reconstruction [192].

\subsubsection{4-D CT Lung Imaging}

Respiratory motion degrades anatomic position reproducibility during imaging, necessitates larger margins during radiotherapy planning and causes errors during radiation delivery. CT scans acquired synchronously with the respiratory signal can be used to reconstruct 4-D CT scans, which can be employed for 4-D treatment planning. This approach explicitly accounts for the respiratory motion and provides an estimate of the intrathoracic tumor motion $[46,81,90,93,104,127,153,174]$.

A 4-D model is generally built with from 4-D CT images. 4-D CT imaging $[41,80,139]$ can be defined as the acquisition of a sequence of 3-D CT image sets over consecutive segments of a breathing cycle. After 4-D CT data acquisition and image reconstruction, a software algorithm retrospectively sorts the images into multiple temporally coherent volumes. Current implementations of 4-D CT utilize phase binning of CT images acquired from patients during normal tidal breathing. Most current sorting methods depend on an externally recorded breathing index associated with each CT slice. An external tracking device such as a real-time position management (RPM) by Varian is one possible breathing index. RPM is an infrared reflective marker placed on the abdomen which is used to provide a signal corresponding to both amplitude and phase of the respiratory signal. Based on the data acquisition time stamps in the image Dicom headers and the correlation signal in the RPM trace, each image can be assigned to a specific respiratory phase of the respiratory cycle. Using a MDCT scanner, $\mathrm{N}$ images are acquired at each location over the entire thorax then sorted into $\mathrm{N}$ respiratory phases [126], as illustrated in Figure 1.6.

In most thoracic 4-D CT studies, ten respiratory phases are imaged and these are typically referred to as phases $\mathrm{P} 00, \mathrm{P} 10, \ldots, \mathrm{P} 90$, where phase $\mathrm{P} 00$ corresponds to end-inhale and phase $\mathrm{P} 50$ corresponds to endexhale. The full set of images provides a movie of the internal anatomical motion resulting from a sampled respiratory cycle and has found use for tumor targeting in radiotherapy treatment planning [97, 168]. Registration of such large data sets requires a computationally efficient image registration algorithm. Furthermore, the image acquisition process renders the resulting thoracic $\mathrm{CT}$ images prone to noise, blurring, and image artifacts Moreover, motion reproducibility assumption with respect to the breathing 


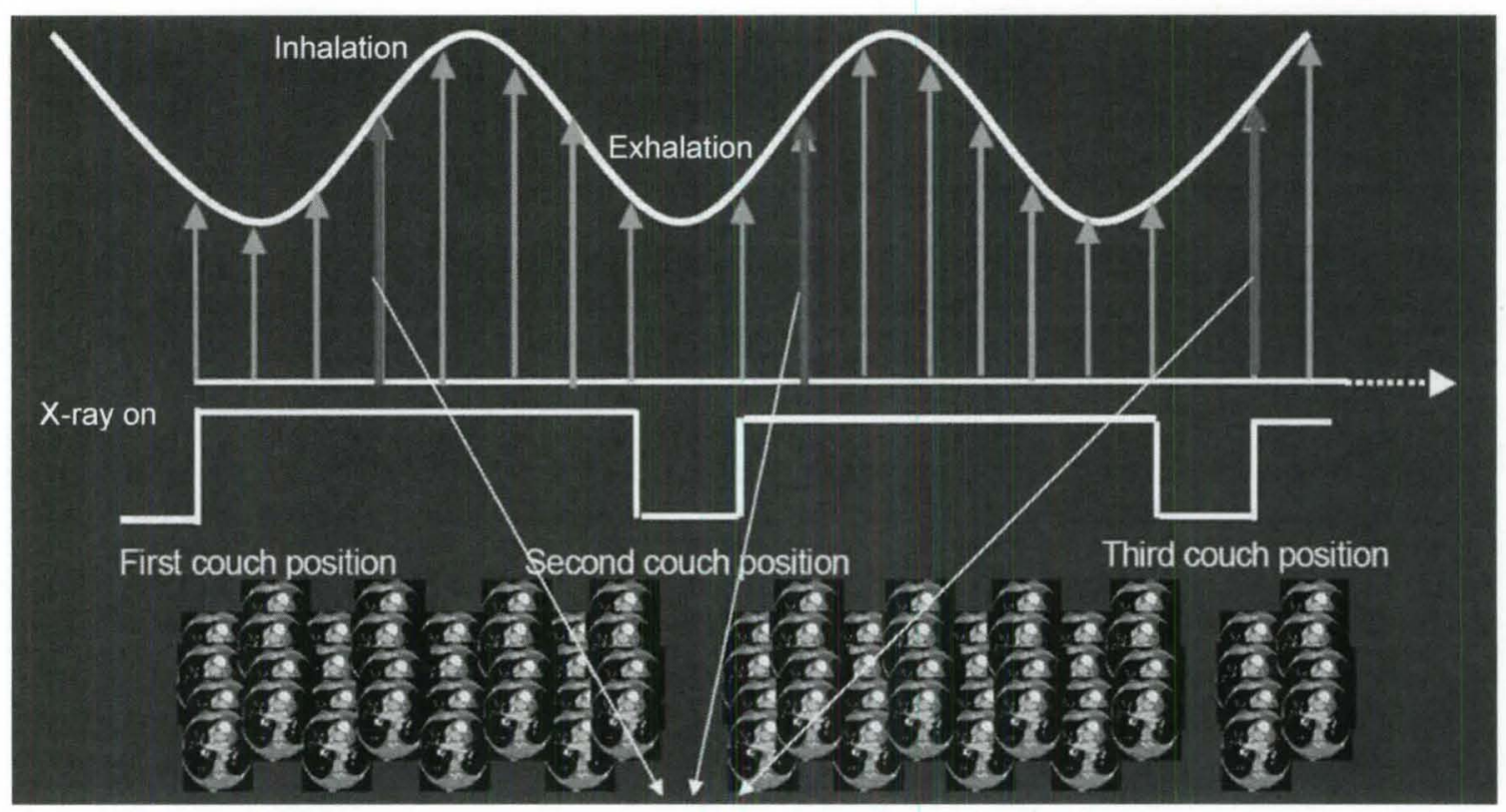

Respiratory 'Bin'

Figure 1.6: .Retrospective 4-D CT Lung Imaging. Images acquired with the aid of respiratory waveform from RPM respiratory gating system in order to bin the image data into different phases.

index and insufficient number of projections per breathing phase for volumetric 3-D reconstruction are potential limitations of 4-D CT. Breath-hold CT images on the other hand, are acquired while the patient holds their breath at either inhale or exhale $[27,42,43,55,89,94,101,156,183]$. Commonly, a static breath-hold scan is at lung volume near FRC or TLC and a 4-D CT dynamic scan is at lung volumes between FRC and FRC+TV. As a result, breath-hold image sets do not suffer from the noise and motion artifacts common to 4-D CT. However, the differences between breath-hold image pairs are considerably greater than those between a sequential pair of images in a 4-D CT. Even though the breath-hold image pairs are relatively "clean", clearly, in comparison to 4-D CT, there is significantly less information available in them.

\subsection{Radiotherapy}

Radiation therapy is a standard technique for cancer treatment where cancer cells are destroyed by highly energetic ionizing radiation. In conformal radiation therapy, high-energy radiation beams from several angles are focused precisely onto the tumor. Ideally these beams overlap exactly in the tumor so 


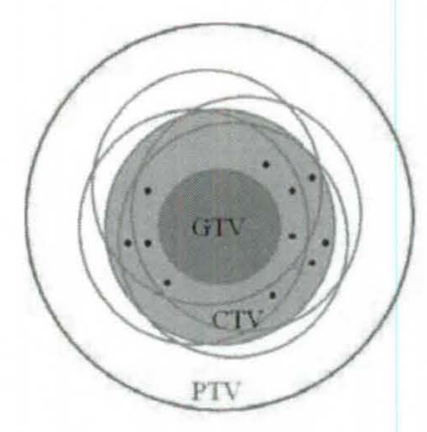

Figure 1.7: Target volumes for radiotherapy planning.

that the tumor receives the maximum dose of radiation while the radiation in the surrounding healthy tissue is minimized. Similarly, intensity-modulated radiation therapy (IMRT) gives radiation therapists the ability to sculpt the edges of a tumor, minimizing damage to adjacent healthy tissue. In IMRT, radiation beams are delivered in approximately 300 different segments, focusing the radiation on the tumor. Dose modulation allows different areas of a tumor or adjacent tissues to receive different doses of radiation. In image-guided radiation therapy (IGRT), repeated imaging scans during treatment are performed to identify changes in a tumor's size and location due to treatment and to allow needed adjustments in the position of the patient or the planned radiation doses.

Nearly all patients receiving thoracic RT develop some degree of lung injury. Irradiating healthy tissues can cause cancer and lead to organ failure due to cell damage. In fact, pulmonary injury and toxicity is the dose-limiting factor in thoracic RT for lung cancer. Vitally important organs that are especially sensitive to radiation are called risk organs, such as the heart and bronchi in the case of lung tumor radiation treatment. These organs should be spared from radiation. Besides beam positioning, therefore, major aspects of radiation treatment planning are determination of risk organs, the target volume that should be treated, and the surrounding healthy tissue. Because of patient movement, such as breathing motion, inaccurate patient positioning and organ motion, the patient anatomy and position may vary during the course of radiation therapy [183]. Due to these motions the actual received dose distribution in the target volume is in fact lower than the planned dose distribution whereas the surrounding healthy tissue ends up receiving some unnecessary radiation.

As shown in Figure 1.7, three regions are defined for target volume definition. The volume containing the tumor is called the gross tumor volume (GTV). The clinical target volume (CTV) is defined to be the 
GTV plus a margin to allow for suspected tumor involvement. The planning target volume (PTV) is defined to contain the CTV plus a margin to account for patient movement or organ motion [183]. Therefore PTV contains healthy tissues that ideally should not be treated.

\subsubsection{Stereotactic Radiation Therapy}

Stereotactic radiation is a specialized form of external beam radiation therapy which makes use of focused radiation beams to target a well-defined tumor using high resolution imaging scans. There are two types of stereotactic radiation treatments. Stereotactic radiosurgery (SRS) involves use of a single or several stereotactic radiation beams in treatment of brain or spine tumors or brain metastases from other cancers. SRS can be used to treat small tumors with well-defined boundaries. A specially designed coordinate-system is used in Stereotactic body radiation therapy (SBRT), for the precise localization of tumors in the body in order to treat it with limited but highly accurate treatment beams. In SBRT, a single high dose radiation treatment or a few fractionated radiation treatments are applied and is typically used to treat tumors that are more likely to move with the normal motion of the body, such as for example tumors of the lung or the liver, and therefore cannot be targeted as accurately as tumors within the brain or spine[79].

The major advantage of SBRT is that it delivers the right therapeutic dose to the tumor in a shorter amount of time than traditional treatments - typically in 10 days or less as opposed to 6 to 11 weeks. With SBRT, treatments are applied with high accuracy in order to limit the effect of radiation on healthy tissues.

\subsection{Deformable Image Registration}

Image registration (IR) problems arise in the context of many different fields. Regardless of the precise context, the IR problem involves description of the relationship between two or more images. Indeed, IR is the process of finding a point-to-point pixel (voxel) mapping (correspondence) between a set of images (Figure 1.8). This desired mapping (correspondence) should describe the location of each pixel (voxel) in the first image relative to the other images(s). When the mapping contains deformations, one speaks of deformable image registration (DIR). The output of DIR is the deformation vector field, indicating the correspondence between each voxel in the first image and a corresponding voxel in the second image. The 


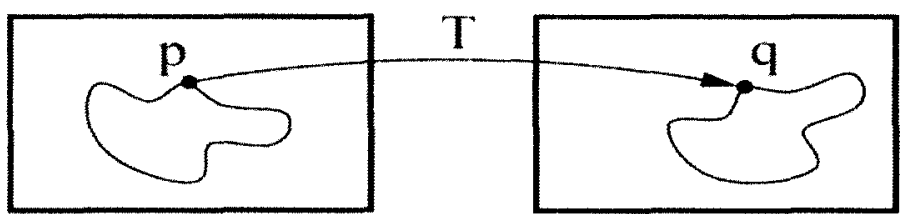

Figure 1.8: Image registration is the task of finding a spatial transformation which maps one image to another [71].

deformation can be applied to the moving image $I_{M}(\xi)$ in order to simulate the reference image $I_{R}(\xi)$ (warping), such that all corresponding voxels have the same coordinates in both images. Registration is the problem of finding a displacement $\boldsymbol{u}(\xi)$ that makes $I_{M}(\xi+\boldsymbol{u}(\xi))$ spatially aligned to $I_{R}(\xi)$. An equivalent formulation is to say that registration is the problem of finding a transformation $T(\xi)=\xi+u(\xi)$ that makes $I_{M}(\boldsymbol{T}(\xi))$ spatially aligned to $I_{R}(\xi)$. The quality of a DIR can be visualized by computing a difference image between reference and warped image, (this metric will be adopted in Chapter 4). In fact, the quality of alignment is defined by a distance or similarity measure $(\delta)$, such as the sum of squared differences (SSD), the correlation ratio (CR), or the mutual information (MI) measure. Since this problem is ill-posed for non-rigid transformations, a regularization term $\mathcal{R}$ is often introduced that constrains the possible transformations. Commonly, registration is formulated as an optimization problem in which the cost function $(\mathcal{C})$, or metric, is minimized so as to find the transformation that provides maximum similarity (or minimum distance) between the reference and the warped image:

$$
\begin{gathered}
\widehat{\boldsymbol{T}}=\underset{T}{\arg \min _{T} \mathcal{C}\left(\boldsymbol{T} ; I_{R}, I_{M}\right), \quad \text { with }} \\
\mathcal{C}\left(\boldsymbol{T} ; I_{R}, I_{M}\right)=-\mathcal{S}\left(\boldsymbol{T} ; I_{R}, I_{M}\right)+\gamma \mathcal{R}(\boldsymbol{T})
\end{gathered}
$$

where $\gamma$ weighs similarity against regularity (smoothness). To solve the above minimization problem, there are basically two approaches: parametric and nonparametric which will be further explained in Chapter 2 . Figure 1.9 shows the general components of a parametric registration algorithm. The input images are resampled into different resolutions. Registration of the lower resolution images requires less memory and computational time. The higher resolution images preserve the local details of the anatomical information, but will require significantly more memory and compute time. Commonly, a multi-resolution strategy is employed to speed-up registration and to make it more robust. The transform box in Figure 1.9 defines how 


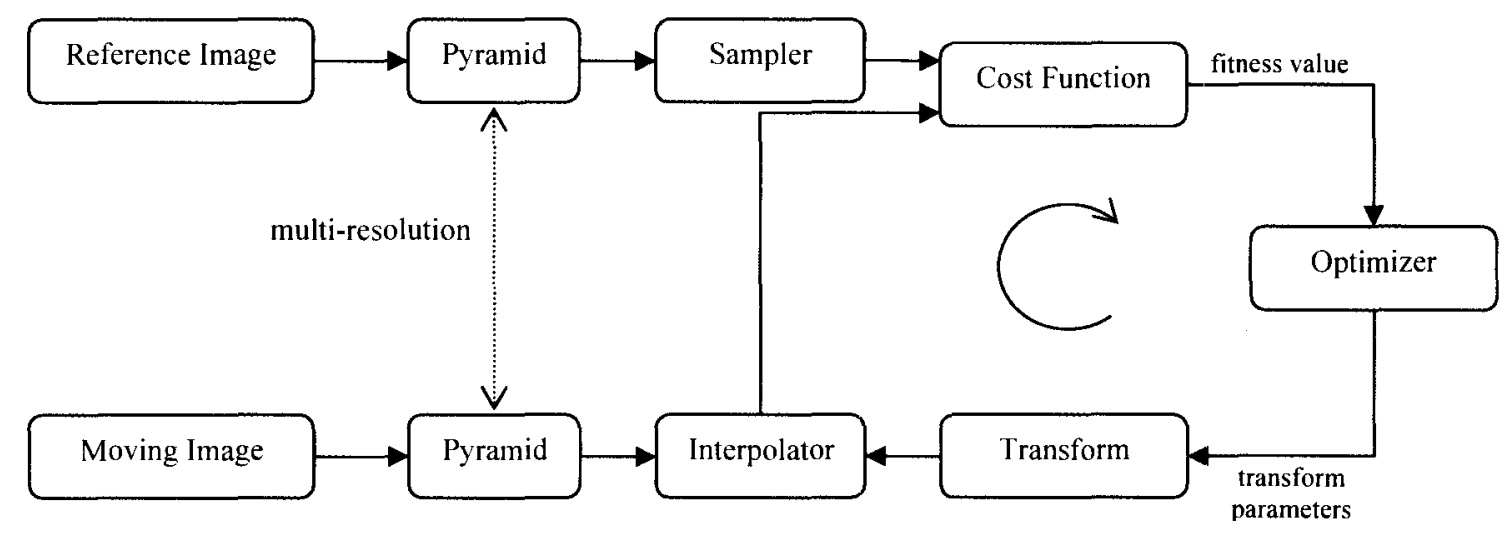

Figure 1.9: The basic components of the image registration framework [71, 83].

one image can be deformed to match another. The transformation can be rigid which can be described by 6 parameters ( 3 translations and 3 rotations). It can also be an affine transformation with 12 parameters ( 3 translations and 9 shear/scaling) for a whole image or it may be a non-rigid registration. The interpolator is used to evaluate the template image intensities at non-integer positions. The cost function component may consist of a single metric such as similarity measure based on geometric and intensity approaches or a compound function with other constraints depending on models that are employed. The cost function measures how well the reference image is matched to the transformed moving template image and is optimized by the optimizer over the search space defined by the parameters of the transform.

\subsubsection{Thoracic CT Registration}

Registration of thoracic CT images is of particular interest to the medical community and to this end a variety of techniques have been proposed. Most often, methods for chest CT registration employ elastic registration and typically include domain-specific modifications to standard approaches $[25,51,76,105$, $108,119,125,148,167]$. There are probably four reasons for registration of CT lung scans [148]:

I. Matching a CT scan to another scan of the same patient from a different modality, typically a PET scan (though with the advent of hybrid PET/CT scanners, there is little need to perform this operation). 
II. Intra-patient registration: matching follow-up CT scans to pre-treatment CT of the same patient for effective visual or automatic comparison to detect or quantify interval change and/or monitor response to therapy.

III. Intra-patient temporal registration: matching a temporal sequence of 3-D thoracic images acquired during respiration in order to quantify motion, deformation, and regional respiratory function.

IV. Inter-patient matching, possibly to an atlas to guide segmentation or to detect deviations from normal appearance.

The multi-modality registration (I), follow-up study (II) and intra-patient 4-D study (III) are within the scope of this research.

Given the growing amount of research dependent on accurate registration of thoracic CT images, the benefit of a DIR algorithm designed to account for the many challenges and peculiarities associated with this problem is clear. Since lung motion varies from apex to base and ventral to dorsal, and is nonhomogeneous, it is desirable to use non-rigid transformations in order to perform temporal registration of the data. In any application, the proper registration method is a compromise between the computational time and demands for accuracy and robustness, especially in the presence of large (ventilation) deformations, pathology, and noise.

\subsection{Regional Ventilation}

Performing DIR to determine regional ventilation essentially has the goal of quantifying local gas exchange inside the lungs. Since the computed ventilation map provides a metric for lung functionality, accurate assessment of regional ventilation as a way to quantify the effects of illness or radiation on lung tissue is essential for the diagnosis and evaluation of pathological conditions. Lung function is not uniform; there are wide ranges of ventilation (and perfusion) levels throughout the lung. Variations in ventilation inside the lung is affected by many factors such as airway geometries, tissue mechanical properties, breathing rate, posture, and so on. Pulmonary ventilation is difficult to measure with physiologically meaningful and quantitative values in three dimensions. Nuclear medicine imaging methods, such as positron emission tomography (PET) and single-photon emission computed tomography (SPECT), provide estimation of regional ventilation by imaging spatial distribution of inhaled gas or aerosol as tracer [59, 


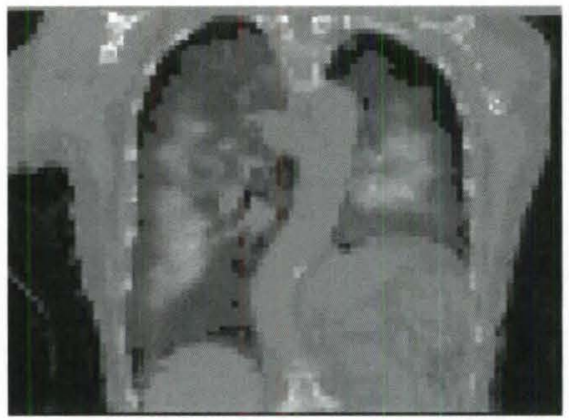

Figure 1.10: SPECT ventilation map image; the bright areas indicate where ventilation is high while the dark areas indicate where ventilation is poor.

$109,158,182]$. However, PET isotopes require an on-site cyclotron with a gas delivery system due to the relatively short half-lives of isotopes limiting their use for research studies. On the other hand, Technetium labeled radiopharmaceutical agent aerosols in SPECT are known to produce artifacts due to airway deposition, rendering them unsuitable for quantitative use. In Oxygen-enhanced magnetic resonance imaging (MRI), images are acquired before and after a simple change in the inspiratory oxygen concentration. The subtraction images are claimed to represent ventilation [39]. Hyperpolarized noble gases, such as xenon or helium, have been utilized to provide paramagnetic contrast for MR ventilation imaging $[4,10,54,145]$. However, MR techniques for ventilation imaging require tracer gases and hyperpolarizer equipment, which limit the availability of these methods. Additionally, the degree of hyperpolarization is time dependent and the resulting images do not yield accurate quantitative physiological values. Multi-detector row CT (MDCT) is able to quantify pulmonary function with a higher resolution despite the ionizing radiation. With inert and non-radioactive xenon gas as a contrast agent, MDCT yields good estimation of regional ventilation by measuring wash-in and wash-out time rates over multiple breathes $[23,58,64,99,145]$. Another disadvantage of the technique is that the requirement for temporal sampling of the wash-in phase limits the spatial coverage of this technique to the axial field of view of the CT scanner and increases the subject's radiation dose. In comparison to MRI, both of those factors limit its use in human subjects. Ideally, a more broadly available, inexpensive, high resolution quantitative ventilation imaging method is still needed. 


\subsubsection{Regional Ventilation from Image Registration}

4-D CT is used routinely as part of standard clinical care for patients suffering from lung cancer [41, 81 , 95, 168, 174, 191]. Although 4-D CT has increased radiation dose, it has high resolution, relatively low cost, and short scan time. However, very relevant to this research, since in 4-D CT spatio-temporal motion is available, it may be used to derive measures of ventilation in patients. The observed spatio-temporal changes in the Hounsfield Unit (HU) in thoracic 4-D CT images reflect the changes in air content of the lungs due to respiration.

Previously, a few investigators have studied the relation between estimates of regional lung expansion and local lung ventilation from 4-D CT as a measure of pulmonary function $[35,56,57,137,160,162]$. Two classes of metrics have been used in CT-based ventilation imaging: Hounsfield Unit (HU) change and Jacobian (ratio of volume) of the deformation field. Both metrics are based on image-registration-derived deformation fields and are based on the assumption that regional ventilation is proportional to the regional volume/density change. Although Jacobian shows better correlation with pulmonary function scans, clear discrepancies have also been reported by several researchers $[20,34,190]$. One of the distinguishing aspects of this research is that physiologic validation of 4-D X-ray CT based ventilation imaging in patients is also included. To date, such validations have only been reported in few studies $[20,21,34,190]$. Using novel DIR techniques proposed in this dissertation, as well as using novel indices of regional pulmonary mechanics and function, we show that we can significantly improve on these previously reported physiologic validations.

\subsection{Application and Significance of Proposed Work}

Many disease or injury conditions can cause biomechanical or structural property changes that can alter lung function. Lung elastic recoil plays a crucial role in breathing [179]. Furthermore, a variety of important diseases involve significant alterations in lung tissue deformation and mechanics $[9,159]$. For example, elastic recoil is markedly elevated in pulmonary fibrosis [195] and surfactant deficiency [100] and can be greatly reduced in emphysema [53]. In fact, virtually all pulmonary diseases of either the obstructive or restrictive classification involve some abnormality of lung tissue mechanics [159]. While the mechanical changes associated with the change of the material properties originate at a regional level, they are largely 
asymptomatic and invisible to global measures of lung function until they have advanced significantly and have aggregated. Therefore it would be desirable to have objective methods with which to evaluate and follow the progression of disease based on measures of regional mechanics.

In the realm of radiation therapy of patients suffering from lung cancer, determining patterns of lung motion prior to radiation treatment as well as predicting treatment toxicity and efficacy can both be clinically relevant. By determining regional lung elasticity and function, radiation treatment planning may be tailored to each patient. This functional information can be used to achieve conformal-avoidance radiation treatment planning. Some dose escalation studies for NSCLC found higher radiation dose associated with improved overall survival [85], while other studies demonstrated the feasibility of minimizing radiation dose to functional lung tissue $[72,194]$. Therefore, if the risk of lung injury can be reduced for all patients, then a greater fraction of NSCLC patients can benefit from higher radiation dose. Having objective methods with which to assess regional lung function, radiation treatment doses can potentially be escalated, thus improving tumor response and local control. Additionally, pulmonary toxicity has the potential to be more accurately predicted and prevented [33], i.e. optimizing radiation beam geometry to preferentially pass through dysfunctional regions of lung and maximally spare regions of normal lung function as well as meaningfully quantifying the radiation toxicity risks by separating normal and dysfunctional regions of the lung in the dose volume histogram.

In this research, we present a technique that uses 4-D CT respiratory gated images of the lung, along with a novel deformable volumetric optical flow method (MOFID), to make regional estimates of lung tissue function and mechanics. We propose to validate our method using expert-defined landmarks and tomographic SPECT ventilation and perfusion images of lung.

The major contribution of our work includes:

1. A new multi-scale optical flow framework with lung-specific assumptions (MOFID) that has the advantage to provide the possibility to enforce physical constraints on the estimated motion field from 4-D CT images of lung.

2. A new elastic registration method to estimate large deformations, such as registration of multimodality images of lung and registration of early and late post-treatment lung images with the pretreatment lung images in order to evaluate the effectiveness of the treatment. 
3. A new mechanical index for assessment of regional lung function as ventilation metric.

4. A physiologic accuracy validation and correlation of the 4-D CT derived quantitative images with SPECT ventilation and perfusion scans, in a population of seven non-small cell lung cancer patients undergoing radiation treatment.

The overarching goal of our study is to design a planning tool for RT planning which would be useful for tracking the progression of radiation induced toxicity to surrounding normal tissue during RT, and can be used to evaluate the effectiveness of treatment.

\subsection{Organization of the Dissertation}

The rest of this dissertation is organized as follows:

Chapter 2 provides a review of well-known and well-established image registration methods. We give an overview of both generic registration algorithms and lung specific registration methods. The review examines the strengths and weaknesses of previously reported approaches.

Chapter 3 introduces the fundamental assumptions and the formulation of a new deformable registration framework (MOFID). We provide details on how the proposed framework enforces lung specific physical constraint in the calculated deformation map from 4-D CT thoracic images.

Chapter 4 discusses a newly proposed elastic registration method for performing large deformation registrations - this technique which combines global statistical intensity information with local image feature information may be used to register the early or late post-treatment lung images with the pretreatment lung images. The same framework may also be used to register multi-modality images (e.g., CT and SPECT).

Chapter 5 introduces and formulates regional mechanical strain as a novel ventilation metric for quantitative measurement of regional pulmonary function. The relation between Jacobian of deformation which has previously been used by several authors with mechanical strain is derived.

Chapter 6 reports on the physiologic accuracy and correlation of the proposed regional pulmonary function measures from radiotherapy treatment-planning thoracic 4-D CT images in a cohort of seven NSCLC patients with tomographic SPECT ventilation and tomographic SPECT perfusion images.

Chapter 7 provides concluding remarks and proposes directions for future research. 


\section{CHAPTER 2}

\section{REVIEW OF LITERATURE ON DEFORMABLE IMAGE REGISTRATION}

\subsection{Introduction}

Imaging of regional pulmonary function (ventilation or perfusion) could be used for functional avoidance in lung cancer radiotherapy and would also further our understanding of pathophysiological characteristics of pulmonary disease and injury conditions. As discussed in section 1.6, many methods have been used in evaluating the function of the lung. These include nuclear medicine imaging (PET and SPECT), hyperpolarized noble gas MRI, and Xe ventilation CT. These techniques have disadvantages such as low resolution, high cost, long scan time, high radiation dose, and/or low accessibility. Since 4-D CT is standard for clinical care for patients diagnosed with lung cancer and is widely available, it can also be used to derive measures of ventilation in addition to functional avoidance. However, ventilation computation and treatment evaluation involves deformable image registration (DIR). DIR is mainly applied in conjunction with image-guided radiation therapy which involves an image-based monitoring of changes in the shape and position of organs and tumor during treatment. The goal is to reduce treatment margins, allowing safe dose escalation, and improving patient treatment. In this chapter, well-known and well-established methods stressing both generic registration algorithms and lung specific registration methods is surveyed. For lung specific techniques, we will describe the way that they take into account nonlinear motions, time-varying boundary conditions during breathing, and changes in intensities due to inflation.

If the nature of the transformation is known a-priori, a registration method can always be decomposed into three major components [98]: the problem statement, the registration paradigm, and the optimization procedure. Problem statement refers to the imaging techniques involved and to the subject to be registered. The registration paradigm determines the nature of the registration and the transformation. It comprises feature detection, feature matching, transform model estimation and image resampling and transformation. 
The optimization procedure alters the parameters of the transformation until a predefined metric reaches an ideal value.

\subsection{Deformable Image Registration Methods}

Thoracic registration methods comprise an extremely wide range of algorithms and are difficult to categorize. In the broadest sense, methods can be split into two main classes: I) Landmark based methods and II) Intensity based methods.

Intensity based methods as intrinsic methods rely on image content and are the main focus of this review primarily due to their independence from the undesirable and required user interaction associated with traditional landmark based techniques. Landmark based methods would be briefly reviewed since it is possible to incorporate them into an intensity-based method $[74,125,129,156]$.

I. Landmark based Methods: Landmark based registration requires knowledge of the location of predefined landmark points in both the reference and target images. They should be homologous, i.e. each landmark in one image is related to its corresponding feature in the other image. The image pairs are then registered based on the motion of the landmarks. In cases where the image motion can be identified by a few parameters, such as an affine motion, the entire displacement field can be determined by only a few landmarks. For more general cases, a displacement field may be computed by interpolating between the known landmarks' displacements.

In thoracic images the landmark based approach uses corresponding anatomical features to define image transformations and takes advantage of segmentation algorithms for delineating the different substructures constituting the pulmonary anatomy, e.g., differentiation of the whole-lung from the chest wall $[50,132,171]$, lobe/fissure detection [36], bronchial structure extraction $[63,92,125,187,196]$, and segmentation of lung vasculature from the parenchyma [50]. Estimation of lung motion via matching of corresponding landmarks is possible once the airway/vessel bifurcations have been identified. However, warping models such as thin-plate spline [27,63,89], radial basis interpolation with the shift log function [101], and B-splines [125, 156, 196] need to be subsequently applied to permit computation of dense displacement fields. 
The main drawback of landmark based methods is the required human involvement needed to identify the landmarks. For example, an expert needs to identify landmarks such as an anatomical structure, bifurcation of airways or vessels, or a tumor before the registration is performed. Moreover, in order to accurately compute the deformation, landmarks should be uniformly distributed within the lung volume. Many review papers have discussed the specifics of landmark registration $[43,62,89,107,202]$.

II. Intensity based Methods: Intensity based methods determine a point-wise correspondence between all image voxels by computing a displacement field based only on image intensities (Hounsfield Units in case of CT images). An immediate advantage of this approach is the absence of required user interaction. However, determining each point's displacement in a given image set is almost impossible, unless the displacement field has some known structure or characteristics. Typically, an intensity based method is built on one key (physical) assumption and one secondary regularization assumption or technique. The physical assumption describes the motion or flow of the imaged point and is the basis for building a voxel motion model or equation. The most common physical assumption is that voxel values remain constant through time and space. However, for lung images this assumption does not truly work due to fluctuation of the lung volume during the respiratory cycle. Therefore, a varying voxel intensity motion model is required [130]. Precisely, how to model the voxel intensity variations depends on the nature of the variations and the characteristics of the image data.

The physical assumption alone does not generally provide enough information to uniquely determine a displacement field. In fact, the fundamental difficulty inherent to all DIR problems is simply a lack of information. Hence, a regularizing assumption is required to make the DIR problem well-posed. Regularization techniques come either in a local or in a global approach.

Local DIR methods solve for pixel displacements after coupling a physical assumption with a regularization assumption valid only in a local neighborhood of the image domain. The most common local regularization assumption which was introduced by Lucas-Kanade is that the displacement field is constant over the neighborhood [96]. In this case, we need only solve either a linear or nonlinear least squares problem to determine the displacement of each point in the neighborhood.

Global methods place a regularization assumption on the behavior of the displacement field over the whole image domain and can be classified as either parametric or non-parametric. A parametric approach 
models the unknown displacement field with a linear combination of basis-functions. First proposed by Horn-Schunck [66], non-parametric approaches place an extra physical property on the displacement field, such as the required level of smoothness. Both techniques lead to a well-posed problem.

DIR methods also categorize based on the way in which the physical assumption is stated. The mathematical expression of the physical assumption is referred as the motion model which is enforced at a single point. Methods utilizing a differential equation as the motion model are referred to as partial differential equation (PDE) based methods versus nonlinear matching methods which employ a nonlinear algebraic equation as the voxel motion model.

\subsubsection{Constant Intensity Methods}

The constant intensity assumption which assumes that the intensity of corresponding voxels remain the same in a temporal sequence of images - this is one of the cornerstones of DIR techniques, especially the optical flow and intensity matching methods. Since due to respiration, the intensity of voxels (i.e., the HU's) in thoracic 4-D CT change with time, these methods, per se, are clearly ill-suited for temporal registration of lung volumes. The varying intensity methods assume some degree of change in the intensity of corresponding points and are defined and implemented using strategies similar to the constant intensity methods; however, the fundamental difference between the two approaches is that in the varying intensity methods the voxel motion models are adjusted to account for intensity variations.

Most registration algorithms designed to calculate the motion of objects captured by image sequences are referred to as optical flow methods. Optical flow methods provide a fine compromise between computational costs and theoretical adaptability and find voxel correspondences between two images by computing a velocity/motion field describing the apparent motion between them. With $\rho$ denoting the image voxel intensity, PDE based methods model the constant intensity assumption with a differential equation commonly referred to as the optical flow equation (also known as the Horn-Schunck optical flow constraint or the brightness constancy constraint):

$$
\frac{d \rho}{d t}=\frac{\partial \rho}{\partial t}+\nabla \rho \cdot v=0
$$

Several authors have previously published review papers and performed comparison of previously published methods $[7,8,91,103,115,180]$. The aforementioned constant intensity assumption in the 
image sequence in optical flow is also common to algorithms that use the sum of squared difference (SSD) similarity metric between two images:

$$
\operatorname{SSD}\left(I_{R}, I_{M}\right)=\int_{\Omega}\left[I_{R}(\xi)-I_{M}(\xi)\right]^{2} d \Omega
$$

where $\Omega$ is the volume over which the motion is to be estimated. The optical flow equation is not adequate to uniquely determine a velocity field, hence a regularization assumption is needed. Global non-parametric strategies involve imposing an additional regularization constraint on the behavior of the displacement field over the entire image domain. Horn-Schunck optical flow [66] is based on Equation (2-1) and the additional regularizing assumption that neighboring voxels move similarly relative to one another. This smoothness assumption implies that the velocity field does not vary abruptly in space, and as a consequence, the gradients of the velocity components should remain small. Horn-Schunck combines both the optical flow equation and the smoothness assumption into a penalty function which is numerically optimized to produce dense deformation fields between successive images:

$$
\min _{v} \int_{\Omega}\left(\rho_{t}+\nabla \rho \cdot v\right)^{2}+\alpha^{2} \sum_{i}\left\|\nabla v_{i}\right\|^{2} d \xi
$$

where $\alpha$ is a parameter determining the weighing of the smoothness term in comparison to the optical flow constraint data term. In comparison to local methods which produce sparse displacements, a significant advantage of global methods - such as Horn-Schunck - is that the computed displacement fields are dense. Indeed, dense displacement fields are desirable in deformable registration of thoracic 4-D CT images in order to permit subsequent computation of mechanical indices of function. Generally, optical flow methods work well when voxel displacements are small, and accurate approximations to image derivatives are available [8]. Basically, large displacements translate into poor approximations for $\frac{\partial \rho}{\partial t}$ which can cause the discretized optical flow equation to become invalid. Therefore, PDE based methods are generally not well equipped to handle large displacements.

Rather than enforce a global constraint, local methods produce displacement field for neighborhoods of the image domain. The Lucas-Kanade method [96] is based on the assumption that the voxel velocity is constant on a neighborhood around a point $\xi$ in the reference image. In Lucas-Kanade, the following weighted least squares problem is posed for determination of the velocity field in image neighborhoods:

$$
\min _{v} \int_{\Omega} \delta_{\sigma}\left(\frac{\partial \rho}{\partial t}+\nabla \rho \cdot v\right)^{2} d \xi
$$


where $\delta_{\sigma}$ is a Gaussian centered at $\xi$ with standard deviation $\sigma$. This Gaussian serves as a window function that isolates the neighborhood around a point $\xi$. The Lucas-Kanade method is robust to image noise because of convolution of the optical flow equation with the Gaussian $\delta$. Similar to the global methods, this method does not yield accurate results when voxel displacements are too large. The displacement field produced by a local method may contain gaps in place where the results are considered unreliable. A filling-in scheme, such as interpolation, is necessary to provide a complete or dense displacement field.

Some authors have adopted combined local-global (CLG) optical flow which was originally derived by Bruhn et al. $[15,16]$ to take advantage of the benefits of both global and local approaches for deriving a dense deformation field. In CLG the traditional optical flow equation is substituted with the Lucas-Kanade formulation:

$$
\min _{v} \int_{\Omega} \int_{\Omega} \delta_{\sigma}\left(\frac{\partial \rho}{\partial t}+\nabla \rho . v\right)^{2} d \zeta+\alpha^{2} \sum_{i}\left\|\nabla \mathcal{V}_{i}\right\|^{2} d \xi
$$

Nonlinear methods utilize a nonlinear algebraic equation, equivalent to the integrated form of the PDE optical flow equation $[87,94,161]$, as the voxel motion model:

$$
I_{M}(\xi+d(\xi))-I_{R}(\xi)=0
$$

In either a local or global framework, application of the integrated optical flow equation does not require approximations to image derivatives. In this sense, nonlinear methods are better suited for handling large displacements and are not as susceptible to image noise.

Nonlinear methods couple the pixel motion model with a regularization assumption and then pose an optimization problem; however, the optimization problems are now nonlinear and generally non-convex. Thus, one needs to deal with local minima and the higher computational costs required for solving the optimization problem. Additionally, some nonlinear methods require evaluation of the image at non-integer locations. Since images are discrete, some type of continuous image representation becomes necessary.

The most popular strategy for creating a continuous image representation is to use B-spline interpolation.[169]. B-splines are piecewise polynomials of degree $n$ (tri-cubic splines are commonly used, $n=3$ ). In particular, B-splines provide a smooth and computationally efficient representation of an image with interesting mathematical properties: compact support, continuous $(n-1)^{\text {th }}$ derivative. They are mostly described by a free-form deformation (FFD) model $[69,94,135,144,166]$. Alternatives to a Bspline representation include the nearest neighbor strategy or bi-linear interpolation [107] 
Two main difficulties of the thoracic CT IR problem are image noise and the large image dimensions. Since the nonlinear methods are computationally costly and that the typical solution route for most PDE methods involves solving a linear system of equations, a PDE approach is more feasible. The only concern is the susceptibility of PDE methods to image noise. However, data pre-processing involving smoothing and application of the CLG approach offers a solution for incorporating noise robustness into the problem formulation.

\subsubsection{Varying Intensity Methods}

To account for temporal variations in voxel intensities, the varying intensity methods require design of an adaptive motion model which considers the nature of the underlying intensity variations [130]. Given an appropriate motion model, it may be incorporated into one of the aforementioned optical flow framework and subsequently an IR method can be formulated by applying any of the regularization strategies described in $[14,44,48,119,152,180]$.

Haussecker et al. [60] proposed a general framework to build a varying brightness model by adjusting the optical flow equation to account for brightness variations:

$$
\frac{\partial \rho}{\partial t}(x(\xi, t), t)+\nabla \rho . v(x(\xi, t), t)=b(\xi, t, a)
$$

where $\boldsymbol{a}$ is a set of parameters required by the brightness model. Equation (2-7) is referred to as the generalized brightness change constraint equation. The same sort of idea applies to a matching method framework $[130,163]$ :

$$
\rho(x(\xi, 0), 0)+d(\xi)=b^{\prime}(\xi, t, \boldsymbol{a})
$$

Accounting for general intensity variations demands calculation of more unknowns and added computational complexity; therefore, one can apply a specific varying intensity voxel motion model. The most commonly employed varying intensity motion model is based on the conservation of mass (continuity) principle:

$$
\rho_{t}+\nabla \rho . v+\rho \operatorname{div}(v)=0
$$

The conservation of mass principle is applicable in situations where the material imaged undergoes local changes in density while overall the total mass of the material is conserved $[26,32,44,134,184]$. Given the fact that the mass of the lung is conserved, the conservation of mass equation is the logical 
choice for the thoracic CT varying intensity motion model $[18,52,116,118,119,198]$. Further, it can be shown that the density of the object imaged in X-ray CT is related to Hounsfield Unit and therefore in (2-9) density and intensity are interchangeable.

Since calculating divergence of an unknown displacement field requires information from neighboring points, it would be difficult to incorporate mass conservation into such methods. Global differential methods - similar to the Horn-Schunck optical flow method - however may be adopted by augmenting the constant intensity model with the mass conservation model. The models described in $[18,44,117,184]$. all use mass conservation in their compressible optical flow formulation, though as will be seen, in addition to differences in the adopted regularization approach, other distinguishing factors exist.

\subsection{Deformable Lung Registration Methods}

The compressible nature of lung tissue which results in varying voxel intensities has hampered 4-D CT thoracic image registration and has been the root of significant challenges. To date, a number of papers have proposed techniques for non-rigid lung motion estimation [17, 55, 105, 108, 136, 198]. Previously proposed techniques most often have employed elastic registration with specific modifications $[25,76,105$, $108,125,148,167]$, such as incorporation of mass conservation [51, 198], or employing respiratory lung motion hysteresis [11]. A useful classification scheme for the intensity and geometric lung registration techniques is based on the two common quantitative measures similarity and smoothness $[98,108,146$, $148,167,180,202]$.

Most authors have used the sum of squared differences (SSD) or the sum of intensity differences (SID) for measuring intensity similarities $[11,25,30,38,43,55,56,77,78,89,94,137,146,164,183,187,193]$. While these measures are useful, they do not accurately reflect the variations of the lung density due to breathing. Since local density changes are linearly reflected in the intensity changes in CT imaging [20], a few authors have used cross correlation (CC) as a similarity measure. In addition to assuming a linear relationship between intensity of corresponding points, $\mathrm{CC}$ requires linear shape changes in order to determine accurate point trajectories $[151,183]$. Some similarity measures, such as mutual information (MI) are insensitive to changes in voxel intensity because they are computed from global image statistics $[27,92,106,125,154,155,172]$. In order to take into account changes in the voxel intensity with inflation, 
some authors have used the sum of squared tissue volume difference (SSTVD) as the similarity criterion $[197,198]$. An alternative approach has been to apply the principle of mass conservation; this has now been adopted by a number of researchers $[18,51,52,116,118-121,198]$.

Most proposed models enforce smoothness on the deformation fields. Included are implicit regularization constraints such as symmetry and consistency which are key to several approaches $[24,25$, $40,88,137,147,151]$.

In the lung tracking area, modifications of the original Horn and Schunck optical flow constraint based on the continuity equation, dubbed compressible optical flow (COF), have revealed improved tracking performance $[18,184]$. Castillo et al. [18] proposed the compressible combined local global (CCLG) optical flow to improve the quality of the deformable image registration of the lung tissue. One drawback of this approach is the associated computational costs requiring a parallel implementation. Some studies have also used a preprocessing method to accommodate the original optical flow constraint for lung registration for example, Sarrut et al. proposed a priori lung density modification (APLDM) to account for the density change during deformation on breath-held images [4]. Subsequently, this approach was extended to 4-D CT images [11].

Some authors have taken advantage of the rich set of anatomic features to formulate lung-specific geometric strategies for registration. The feature-based approach uses corresponding anatomical features to define image transformations and takes advantage of segmentation algorithms for delineating the different substructures constituting the pulmonary anatomy, e.g., differentiation of the whole-lung from the chest wall $[50,132,171]$, lobe/fissure detection [36], bronchial structure extraction [63, 92, 125, 187, 196], and segmentation of lung vasculature from the parenchyma [50]. Estimation of lung motion via matching of corresponding landmarks is possible once the airway/vessel bifurcations have been identified. However; warping models such as thin-plate spline $[27,63,89]$, radial basis interpolation with the shift log function [101], and B-splines $[125,156,196]$ need to be subsequently applied to permit computation of dense displacement fields.

Temporal registration methods either use an Eulerian approach or a Lagrangian approach to motion tracking. In the Eulerian approach, all deformations are computed between pairs of neighboring time points, while in the Lagrangian approach, deformations are described with respect to a chosen reference 
frame. Metz et al. [105] surveyed and categorized different medical registration approaches based on their transformation model which either describe an Eulerian or a Lagrangian approach. Although the Lagrangian approach has the advantage of permitting a strict comparison of deformations in successive frames relative to one another, the Lagrangian model causes a bias towards the reference and the need to deal with estimation of larger deformations. Predominantly, existing methods use a Lagrangian model, though for the most part, do not take into account the temporal smoothness of the deformations $[17,78$, $105,136,138,198]$. In recent times, several authors have also adopted temporal smoothness constraints as well $[17,105,120]$

\subsection{Performance Evaluation of Lung Registration Methods}

Evaluation of the computed estimation of registration methods is nontrivial because the ground truth motion (e.g. gold standard) is often not available. Additionally, errors can occur in each step of a registration process, and it is hard to differentiate between registration inaccuracies and actual physical differences in the image content. Even if a gold standard is available, often the uncertainty in the gold standard itself limits the ability to assess true accuracy [185]. The literature proposes many different methods for registration validation $[73,108,167,185,202]$; however, it is difficult to compare the accuracy measured with one method to that of another method due to methodological incompatibilities [185]. It should also be pointed out that although some reported literature confines itself to only one of the validation categories described below, many research groups have used two or more of the validation techniques [167].

One of the quickest methods is visual inspection of the result [185]. Visual inspection of the registration can offer a qualitative idea of the accuracy. Another possibility is to use intensity-based similarity measures, such as cross correlation, before and after registration in order to assess performance of the registration method $[30,177]$.

Analysis of point correspondences is also commonly used to evaluate registration algorithms $[11,19$, $45,61,131,173,187]$. Given the abundant features present within the lung (e.g. vessel and airway bifurcations) seen in CT images, the set of homologous points can be manually annotated by an expert in the different phases of the lung. The difference between the estimated position of the landmark by 
registration method and the actual position of the landmark may then be measured and is referred to as the ground truth discrepancy. A common approach is to report the average error, the root mean square (RMS) error, and the average angular error (AAE) for the calculated motion field. Nonetheless, the manual selection of landmarks is certain to have some inaccuracy while in the case of automated selection of landmarks, error estimation becomes more complicated. This is because we cannot differentiate between the localization error of landmarks due to their inaccurate selection, the matching error, and the error due to the difference between the transformation model and the actual deformation.

While the annotated landmark pairs are used to assess correspondence internal to the lung, overlap assessment of anatomic contours of the lung are used to assess the alignment of lung boundaries. Aligning the boundaries of the lungs correctly is fundamentally expected of all pulmonary CT registration algorithms. The lung boundary is easily defined in CT in most regions, with the notable exception of the mediastinal (central) regions [108]. Some researchers have used manual or automated segmentation to compare the measured tidal volumes with volumes estimated from the calculated displacement fields [37, $38,56,57,175]$. If corresponding anatomic structures may be delineated, common overlap measures such as Jaccard and Dice may be used to assess the quality of overlap for both single and multiple labeled images $[28,47,89,167,201]$. For source and target segmented regions, $\delta$ and $\mathcal{T}$, respectively, the Jaccard coefficient is defined as:

$$
\operatorname{Jaccard}(\mathcal{S}, \mathcal{T})=\frac{|\mathcal{S} \cap \mathcal{T}|}{|\mathcal{S} \cup \mathcal{T}|}
$$

whereas the Dice coefficient is calculated from:

$$
\operatorname{Dice}(\mathcal{S}, \mathcal{T})=2 \frac{|\delta \cap T|}{|\mathcal{S}|+|\mathcal{T}|}
$$

The final figure of merit is the Jacobian of the calculated deformation map to determine physical plausibility of the calculated deformation. In particular, we expect that a deformation should be bijective. Regions where the deformation field is not bijective are commonly referred to as singularities. The determinant of the Jacobian of the deformation field at every point (described further in Chapter 5) specifies whether local expansion or contraction has taken place. Any point where Jacobian is zero is a singularity in the deformation map. Additionally, a negative Jacobian implies a deformation that is physically not permissible since that would imply folding of tissue onto itself. 


\section{CHAPTER 3}

\section{MASS PERSERVING OPTICAL FLOW}

\subsection{Introduction}

This chapter introduces an approach to Deformable Image Registration (DIR) of 4-D thoracic CT data via a novel highly versatile 3-D mass conserving optical flow technique referred to as MOFID for computing lung motion within a variational formulation which uses an Eulerian approach. The energy function for computing optical flow has been designed to address the shortcomings of the existing DIR methods for 4-D thoracic CT.

4-D thoracic $\mathrm{CT}$ is characterized by both tissue motion as well as respiratory induced changes in the CT image resulting from ventilation. Deformable image registration provides a link between the component phase images for extraction of the motion and physiological information. Registration of 4-D thoracic CT images is specifically difficult due to the compressible nature of the lung and the high computational workload required to handle the massive amount of data.

The original optical flow constraint has been modified and provided with some dedicated lung specific constraints to take advantage of both geometric and intensity features of 4-D CT images of the lung. The proposed method (MOFID) is a global partial differential equation (PDE) based technique which enforces the governing intensity constancy assumption in standard optical flow, but in addition has a constraint that ensures intensity gradient constancy in consecutive image frames to allow variations in the gray value and to better track vessel and airway boundaries, mass conservation in consecutive volumetric image frames to ensure that the local lung mass is conserved over the entire imaged region, and spatio-temporal smoothness of the deformation map to regularize the estimated motion in order to estimate smooth deformations of the

lung in space and time. Taken together, these constraints permit highly accurate estimates of the lung motion between different respiratory phases from 4-D CT images. 
In the rest of this chapter, a detailed derivation of the proposed energy function formulation is first provided. Subsequently, the details of multi-scale numerical implementation of the method and the proposed approach to determination of the optimum parameters for the energy function are discussed [117]. Although less accurate, the 2-D planar version of our DIR method is first described [118] before our volumetric DIR technique [119]. A comprehensive validation of MOFID against expert annotated landmarks for 11 4-D X-ray CT lung datasets acquired in human subjects has been reported. Comparison of the MOFID with previously published results is also included in this chapter [120].

\subsection{The Variational Formulation}

In order to derive a variational formulation for the proposed optical flow method, below, the constraints of interest are listed:

1. Ideally, the gray value of a voxel should not change after displacement:

$$
I(\xi+W)=I(\xi)
$$

In this equation, $I$ is the image intensity as a function of space and time, $\xi:=(x, y, z, t)^{T}$, and $W$ $:=(u, v, w, 1)^{T}$ is the displacement vector field between two subsequent images. This is a nonlinear equation in $u, v$, and $w$. Its linearized version yields the well-known optical flow constraint [66]:

$$
I_{x} u+I_{y} v+I_{z} w+I_{t}=0
$$

However the linearization of the intensity constancy assumption is in general an unreliable approximation of the original constraint $[5,110]$. In particular in the case of 4-D thoracic CT images, the displacement between respiration phases does not change linearly along the displacement vector. Therefore the non-linearized equation is adopted and the linearization is postponed to the numerical scheme.

2. In anatomical $\mathrm{CT}$ images, we assume the imaged intensities follow a conservation principle in analogy to mass conservation in fluid flow. In fact, one can show that if it can be assumed that the Hounsfield unit is proportional to density then the two conservation principles are equivalent. Application of the conservation principle to a temporally varying image yields:

$$
\nabla \cdot(I V)+I_{t}=0 \Rightarrow \nabla I .(V)+I(\nabla \cdot V)+I_{t}=0
$$



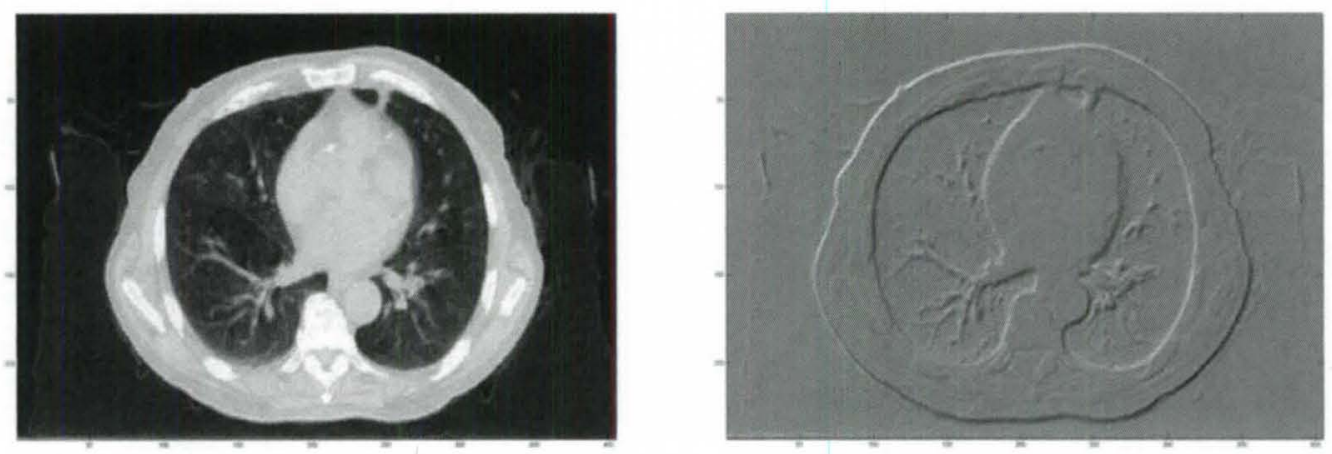

Figure 3.1: An axial CT slice of lung on the left and the corresponding gradient image on the right demonstrating pronouncement of the pulmonary anatomy and bronchial structures - In the proposed implementation, the gradient image is used (Equation (3-5)) to bring to bear the significant utility of this rich feature set in order to improve the optical flow tracking performance.

$$
I_{x} u+I_{y} v+I_{z} w+I u_{x}+I v_{y}+I w_{z}+I_{t}=0
$$

where $V=(u, v, w)$ is the velocity field. Equation (3-4) is a more general constraint for deformable object motion in comparison to the classic Horn-Schunck optical flow constraint equation shown in (3-2).

3. In order to allow variations in the grey value and to help determine the displacement vector by a criterion that favors matching moving edges, a constraint based on image gradients is also incorporated:

$$
\nabla I(\xi+W)=\nabla I(\xi)
$$

Linearization leads to:

$$
\begin{aligned}
& I_{x x} u+I_{x y} v+I_{x z} w+I_{x t}=0 \\
& I_{x y} u+I_{y y} v+I_{y z} w+I_{y t}=0 \\
& I_{x z} u+I_{y z} v+I_{z z} w+I_{z t}=0
\end{aligned}
$$

where double scripts denote second derivatives. This constraint was originally proposed within a variational 2-D optical flow framework by Brox et al. [14]. As shown in Figure 3.1, this constraint takes advantage of the rich set of anatomic features of thoracic CT data to match substructures of the pulmonary anatomy in order to align the bronchial structures in two successive respiratory phases. The quantitative evidence for importance of this term in the energy function will be provided in Section 3.4.2 and Figure 3.10 . 
4. Finally, in order to regularize the solution, spatio-temporal smoothness of the flow field has been adopted. The advantage of this approach proposed for the variational optical flow framework by Weickert et al. [181] is to smooth out the background noise and preserve true motion boundaries:

$$
\left|\nabla_{4} u\right|^{2}+\left|\nabla_{4} v\right|^{2}+\left|\nabla_{4} w\right|^{2}
$$

where $\nabla_{4}:=\left(\partial_{x}, \partial_{y}, \partial_{z}, \partial_{t}\right)^{T}$ and $\mathrm{u}, \mathrm{v}$, and $\mathrm{w}$ are components of displacement along $\mathrm{x}, \mathrm{y}$, and $\mathrm{z}$. The extension of the spatial smoothness to the spatio-temporal domain in general leads to better estimates due to the additional information exchange along the temporal axis. Therefore, the energy functional that penalizes deviations from these model assumptions is formulated as:

$$
\begin{gathered}
E(u, v, w)=\int_{\Omega}\left(E_{D}+\alpha E_{S}\right) d v=\int_{\Omega} \psi\left[|I(X+W)-I(X)+\beta(I \nabla \cdot V)|^{2}+\gamma|\nabla I(X+W)-\nabla I(X)|^{2}\right]+ \\
\alpha\left(\psi\left[\left|\nabla_{4} u\right|^{2}+\left|\nabla_{4} v\right|^{2}+\left|\nabla_{4} w\right|^{2}\right]\right) d v
\end{gathered}
$$

where $\Omega$ is the volume over which the motion is to be estimated. The function $\psi\left(s^{2}\right)=\sqrt{s^{2}+\varepsilon^{2}}$ is a modified $L_{1}$ norm which is convex and yields robustness to outliers in the minimization process. Since $\varepsilon$ is only utilized for numerical reasons, it can be set to a fixed value (we chose 0.001 ). $\beta$ can only be zero or one: zero when the object is incompressible and one when the object is compressible (for the thoracic CT application $\beta$ is always one). In order to obtain a numerical solution, this energy function should be discretized with all image derivatives approximated by finite differences.

\subsection{Numerics}

A minimizer of $E(u, v, w)$ must fulfill the Euler-Lagrange equations:

$$
\left\{\begin{array}{c}
E_{u}-\frac{\partial}{\partial x} E_{u_{x}}-\frac{\partial}{\partial y} E_{u_{y}}-\frac{\partial}{\partial z} E_{u_{z}}-\frac{\partial}{\partial t} E_{u_{t}}=0 \\
E_{v}-\frac{\partial}{\partial x} E_{v_{x}}-\frac{\partial}{\partial y} E_{v_{y}}-\frac{\partial}{\partial z} E_{v_{z}}-\frac{\partial}{\partial t} E_{v_{t}}=0 \\
E_{w}-\frac{\partial}{\partial x} E_{w_{x}}-\frac{\partial}{\partial y} E_{w_{y}}-\frac{\partial}{\partial z} E_{w_{z}}-\frac{\partial}{\partial t} E_{w_{t}}=0
\end{array}\right.
$$

Having adopted the finite difference approximation proposed by Weickert et al. [14, 181] and for better readability, the following abbreviations are used:

$$
\begin{aligned}
& I_{x}:=\frac{\partial}{\partial x} I(X+W), \\
& I_{y}:=\frac{\partial}{\partial y} I(X+W), \\
& I_{z}:=\frac{\partial}{\partial z} I(X+W),
\end{aligned}
$$




$$
\begin{gathered}
I_{\phi}:=I(X+W)-I(X), \\
I_{x x}:=\frac{\partial^{2}}{\partial x^{2}} I(X+W), \\
I_{y y}:=\frac{\partial^{2}}{\partial y^{2}} I(X+W), \\
I_{z z}:=\frac{\partial^{2}}{\partial z^{2}} I(X+W), \\
I_{x y}:=\frac{\partial^{2}}{\partial x \partial y} I(X+W), \\
I_{x z}:=\frac{\partial^{2}}{\partial x \partial z} I(X+W), \\
I_{y z}:=\frac{\partial^{2}}{\partial y \partial z} I(X+W), \\
I_{x \phi}:=\frac{\partial}{\partial x} I(X+W)-\frac{\partial}{\partial x} I(X), \\
I_{y \phi}:=\frac{\partial}{\partial y} I(X+W)-\frac{\partial}{\partial y} I(X), \\
I_{z \phi}:=\frac{\partial}{\partial z} I(X+W)-\frac{\partial}{\partial z} I(X)
\end{gathered}
$$

In the interest of brevity, derivations that relate to the $u$ component of the flow field is only included. Derivations for the $v$ and $w$ component are identical.

Therefore:

$$
\begin{aligned}
& \psi^{\prime}\left[I_{\phi}^{2}+(\beta(I \nabla . V))^{2}+2 I_{\phi}(\beta(I \nabla . V))+\gamma\left(I_{x \phi}{ }^{2}+I_{y \phi}{ }^{2}+I_{z \phi}{ }^{2}\right)\right] \cdot\left(I_{x} I_{\phi}+I_{x} I_{\phi}(\beta(I \nabla . V))+\right. \\
& \left.\gamma\left(I_{x x} I_{x \phi}+I_{x y} I_{y \phi}+I_{x z} I_{z \phi}\right)-\beta(I \nabla . V)\left(1+I_{\phi}\right)\right)-\alpha \operatorname{div}\left(\psi^{\prime}\left[\left|\nabla_{4} \mathrm{u}\right|^{2}+\left|\nabla_{4} \mathrm{v}\right|^{2}+\left|\nabla_{4} \mathrm{~W}\right|^{2}\right] \nabla_{4} \mathrm{u}\right)=0
\end{aligned}
$$

with the iteration variable $W^{k}$ instead of $W, W^{k+1}$ will be the solution of;

$$
\begin{aligned}
& \psi^{\prime}\left[I_{\phi}^{k+1}{ }^{2}+\left(\beta\left(I^{k} \nabla \cdot\left(u^{k+1}, v^{k+1}, w^{k+1}\right)\right)\right)^{2}+2 I_{\phi}^{k+1}\left(\beta\left(I^{k} \nabla \cdot\left(u^{k+1}, v^{k+1}, w^{k+1}\right)\right)\right)+\gamma\left(I_{x \phi}^{k+12}+I_{y \phi}^{k+1^{2}}+\right.\right. \\
& \left.\left.I_{z \phi}^{k+1}{ }^{2}\right)\right] \cdot\left(I_{x}^{k} I_{\phi}^{k+1}+I_{x}^{k} I_{\phi}^{k+1}\left(\beta\left(I^{k} \nabla \cdot\left(u^{k+1}, v^{k+1}, w^{k+1}\right)\right)\right)+\gamma\left(I_{x x}^{k} I_{x \phi}^{k+1}+I_{x y}^{k} I_{y \phi}^{k+1}+I_{x z}^{k} I_{z \phi}^{k+1}\right)-\right. \\
& \left.\beta\left(I^{k} \nabla \cdot\left(u^{k+1}, v^{k+1}, w^{k+1}\right)\right)\left(1+I_{\phi}^{k+1}\right)\right)-\alpha \operatorname{div}\left(\psi^{\prime}\left[\left|\nabla_{4} \mathrm{u}^{\mathrm{k}+1}\right|^{2}+\left|\nabla_{4} \mathrm{v}^{k+1}\right|^{2}+\left|\nabla_{4} \mathrm{w}^{k+1}\right|^{2}\right] \nabla_{4} \mathrm{u}^{\mathrm{k}+1}\right)=0
\end{aligned}
$$

To remove the nonlinearity in $l_{*}^{k+1}$, first order Taylor expansion yields

$$
\begin{gathered}
I_{\phi}^{k+1} \approx I_{\phi}^{k}+I_{x}^{k} d u^{k}+I_{y}^{k} d v^{k}+I_{z}^{k} d w^{k} \\
I_{x \phi}^{k+1} \approx I_{x \phi}^{k}+I_{x x}^{k} d u^{k}+I_{x y}^{k} d v^{k}+I_{x z}^{k} d w^{k} \\
I_{y \phi}^{k+1} \approx I_{y \phi}^{k}+I_{x y}^{k} d u^{k}+I_{y y}^{k} d v^{k}+I_{y z}^{k} d w^{k}
\end{gathered}
$$




$$
I_{z \phi}^{k+1} \approx I_{z \phi}^{k}+I_{x z}^{k} d u^{k}+I_{y z}^{k} d v^{k}+I_{z z}^{k} d w^{k}
$$

where $u^{k+1}=u^{k}+d u^{k}, v^{k+1}=v^{k}+d v^{k}$, and $w^{k+1}=w^{k}+d w^{k}$.

Introducing an abbreviation for the robustness factor $\psi_{\text {Data }}^{\prime}$ and the diffusivity $\psi_{\text {smooth }}^{\prime}$,

$$
\begin{aligned}
\left(\psi_{D a t a}^{\prime}\right)^{k}:=\psi^{\prime}[ & \left(I_{\phi}^{k}+I_{x}^{k} d u^{k}+I_{y}^{k} d v^{k}+I_{z}^{k} d w^{k}\right)^{2}+\left(\beta\left(I^{k} \nabla \cdot\left(u^{k}+d u^{k}, v^{k}+d v^{k}, w^{k}+d w^{k}\right)\right)\right)^{2} \\
& +2\left(I_{\phi}^{k}+I_{x}^{k} d u^{k}+I_{y}^{k} d v^{k}+I_{z}^{k} d w^{k}\right)\left(\beta\left(I^{k} \nabla \cdot\left(u^{k}+d u^{k}, v^{k}+d v^{k}, w^{k}+d w^{k}\right)\right)\right) \\
& +\gamma\left(\left(I_{x \phi}^{k}+I_{x x}^{k} d u^{k}+I_{x y}^{k} d v^{k}+I_{x z}^{k} d w^{k}\right)^{2}+\left(I_{y \phi}^{k}+I_{x y}^{k} d u^{k}+I_{y y}^{k} d v^{k}+I_{y z}^{k} d w^{k}\right)^{2}\right. \\
& \left.\left.+\left(I_{z \phi}^{k}+I_{x z}^{k} d u^{k}+I_{y z}^{k} d v^{k}+I_{z z}^{k} d w^{k}\right)^{2}\right)\right]
\end{aligned}
$$

$$
\left(\psi_{\text {Smooth }}^{\prime}\right)^{k}:=\psi^{\prime}\left[\left|\nabla_{4}\left(u^{k}+d u^{k}\right)\right|^{2}+\left|\nabla_{4}\left(v^{k}+d v^{k}\right)\right|^{2}+\left|\nabla_{4}\left(w^{k}+d w^{k}\right)\right|^{2}\right]
$$

As the only remaining nonlinearity is due to $\psi^{\prime}$, and $\psi$ has been chosen to be a convex function, the remaining optimization problem is a convex problem; i.e., there exists a unique minimum. To remove the remaining nonlinearity in $\psi^{\prime}$, a second, inner fixed point iteration loop is applied. Let $l$ denote the iteration index for this inner loop. The fixed point variables $d u^{k, l}, d v^{k, l}$, and $d w^{k, l}$ are initialized with 0 . Therefore, the linear system of equations in $d u^{k, l+1}$ :

$$
\begin{aligned}
\left(\psi_{\text {Data }}^{\prime}\right)^{k, l}\left(I _ { x } ^ { k } \left(I_{\phi}^{k}\right.\right. & \left.+I_{x}^{k} d u^{k, l+1}+I_{y}^{k} d v^{k, l+1}+I_{z}^{k} d w^{k, l+1}\right)(1 \\
& \left.+\beta\left(I^{k} \nabla \cdot\left(u^{k}+d u^{k, l+1}, v^{k}+d v^{k, l+1}, w^{k}+d w^{k, l+1}\right)\right)\right) \\
& +\gamma\left(I_{x x}^{k}\left(I_{x \phi}^{k}+I_{x x}^{k} d u^{k, l+1}+I_{x y}^{k} d v^{k, l+1}+I_{x z}^{k} d w^{k, l+1}\right)\right. \\
& +I_{x y}^{k}\left(I_{y \phi}^{k}+I_{x y}^{k} d u^{k, l+1}+I_{y y}^{k} d v^{k, l+1}+I_{y z}^{k} d w^{k, l+1}\right) \\
& \left.+I_{x z}^{k}\left(I_{z \phi}^{k}+I_{x z}^{k} d u^{k, l+1}+I_{y z}^{k} d v^{k, l+1}+I_{z z}^{k} d w^{k, l+1}\right)\right) \\
& -\beta\left(I^{k} \nabla \cdot\left(u^{k}+d u^{k, l+1}, v^{k}+d v^{k, l+1}, w^{k}+d w^{k, l+1}\right)\right)(1 \\
& \left.\left.+\left(I_{\phi}^{k}+I_{x}^{k} d u^{k, l+1}+I_{y}^{k} d v^{k, l+1}+I_{z}^{k} d w^{k, l+1}\right)\right)\right) \\
& -\alpha d i v\left(\left(\psi_{S m o o t h}^{\prime}\right)^{k, l}\left[\nabla_{4}\left(u^{k}+d u^{k, l+1}\right)\right]\right)=0
\end{aligned}
$$

Discretization yields a linear system of equations, which can be iteratively solved by Successive Over Relaxation (SOR) [133]. Let $m$ denote the iteration index for the SOR iterations, then the iteration scheme for solving the linear system is: 


$$
\begin{aligned}
& d u_{i}^{k, l, m+1}=
\end{aligned}
$$

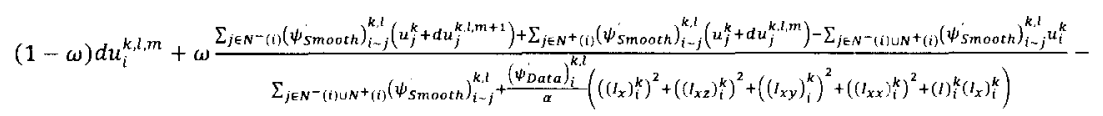

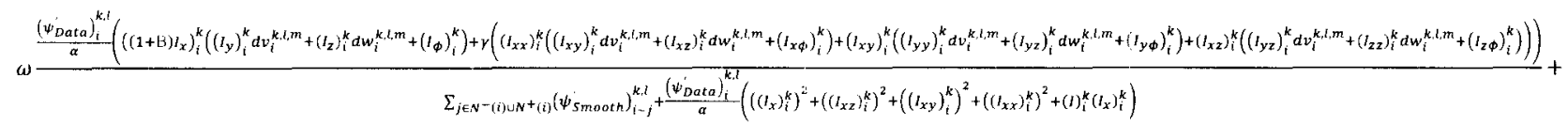

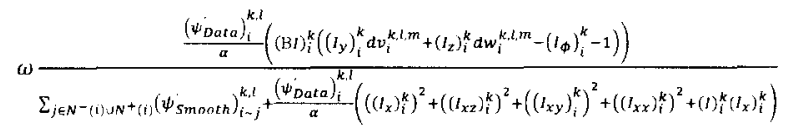

where $\mathrm{B}=\beta\left(I^{k} \nabla \cdot\left(u^{k}+d u^{k, l}, v^{k}+d v^{k, l}, w^{k}+d w^{k, l}\right)\right)$.

Since the proposed method converges to the estimated motion in an iterative form, a multi-scale framework was used to speed up the calculations realizing significant computational savings. A multiresolution strategy helps to improve the computational efficiency as well as to deal with larger displacements which may be encountered due to limited number of imaged respiratory phases [197]. In the multi-resolution framework, both images are first downsampled and then registered. Once the registration has been completed, the deformation field is upsampled and the calculated deformation field is then propagated to the next finer level and used as the initial transformation for that level.

While the proposed method results in accurate lung motion estimation, the number of weighting factorsthat is $\alpha, \gamma$, and $l$ (number of multi-scale level)- and their various combinations is a significant challenge. To find the optimum weights, normalized mutual information (NMI) [157] has been used as a similarity measure between the first frame warped with the calculated motion field and the second frame. NMI is essentially used here as the yardstick to compare the accuracy of the estimated motion for different weighting factors $[22,68,128,157]$. Figure 3.2 shows a similarity calculation between the first warped image and the second image corresponding to two consecutive respiratory phases of POPI-model for five different $\alpha$ values, that is from 10 to 50 (increments by 10), five different $\gamma$, that is from 50 to 210 (increments by 40 ) and for $l=5,6$. 


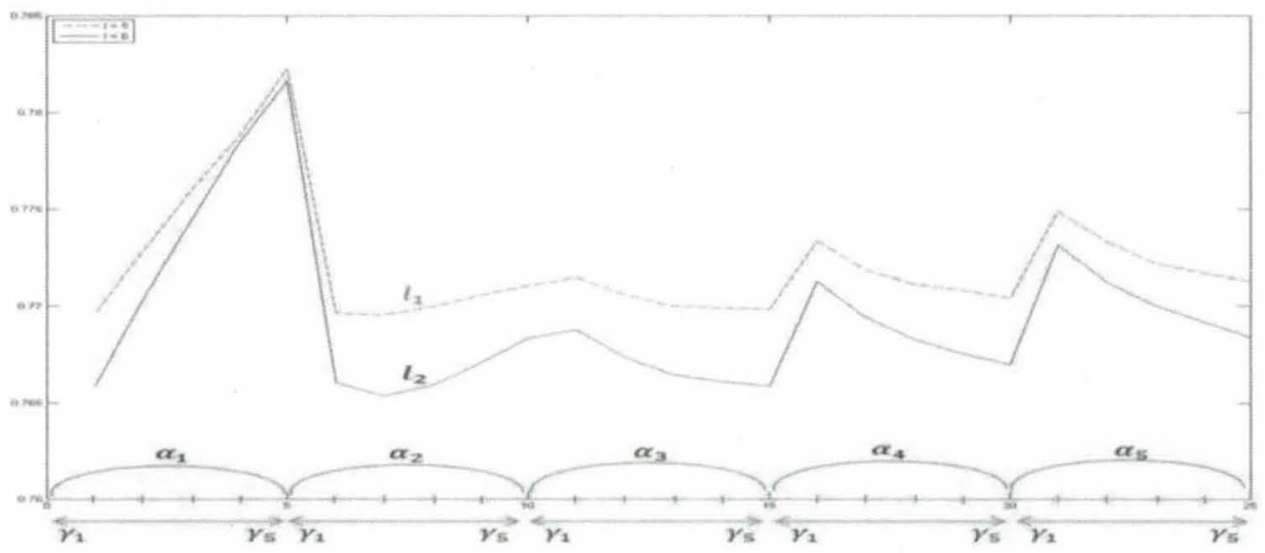

Figure 3.2: Similarity calculation between the first warped image and the second image of the POPI-model data set. This graph shows similarity calculation in terms of five gamma $\left(\gamma_{1} \sim \gamma_{5}\right)$, that is from 50 to 210 in increments of 40 , and five alpha $\left(\alpha_{1} \sim \alpha_{5}\right)$ values from 10 to 50 in increments of 10 , for two different number of levels $l_{1}=5$ and $l_{2}=6$. Note that the smaller the value of the plot, the higher the similarity.

\subsection{Experimental Results}

Though the purpose of the MOFID is to estimate the motion of the volumetric CT images of the lung, a series of 2-D experiments were conducted on both synthetic and real data-sets to test the efficacy of the proposed method.

\subsubsection{Two Dimensional Experiments}

The proposed method has been applied to well-known image sequences such as the symmetric sponge expansion and the Yosemite sequence with cloudy sky, both used extensively by previous authors. The sponge sequence illustrates the scenario under which mass conservation holds while the Yosemite sequence typifies most cases where mass conservation does not hold in 2-D. The algorithm is capable of handling both cases: $\beta=1$ would enforce mass conservation while $\beta=0$ would result in no mass conservation. These results are shown in Figure 3.4. As shown in the color wheel (Figure 3.3) used to visualize flow fields in this study, smaller vectors are darker while the color indicates direction of movement.

For both qualitative and quantitative evaluation of the proposed method, POPI-model data-set of lung deformation has been used [173]. The POPI-model is a 4-D pixel-based and point-validated breathing 


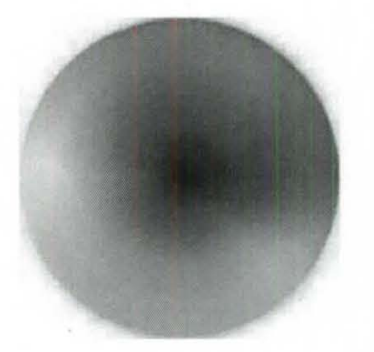

Figure 3.3: Color wheel for encoding estimated motion vectors: smaller vectors are darker while color indicates direction of movement.

thorax model which is used to validate the planar results of our method. The anonymized respirationcorrelated 4-D CT image consists of 10 3-D CT images sampling the entire respiratory cycle. Images were acquired on a 16 slice Brilliance CT Big Bore Oncology configuration (Philips). Breathing correlated information was obtained using associated Pneumo Chest bellows (Lafayette Instruments). Each 3-D image volume is made up of 141 axial slices with a slice thickness of $2 \mathrm{~mm}$. Each slice is a 2-D image with size $512 \times 512$ with an isotropic in-plane resolution of $0.97 \mathrm{~mm}$. Landmarks are defined by experts in all of the 3D CT image volumes that make up the 4-D CT data. Although it is clear that landmarks in general move in 3-D, a subset of them which exhibited planar motion in subsequent frames were chosen in order to validate the proposed methodology. The average number of available landmarks for validation of our method for each of the coronal, sagittal, and axial orientation through-out the breathing cycle was 25,24 , and 22 . Figure 3.5 shows calculated vector field in all three anatomical orientations.

The average computational time to arrive at these results were 38 seconds within a multi-scale framework on a Dell 5160 machine with a quad-core Xeon $3.00 \mathrm{GHz}$ processor. Whereas Figures 3.4 and 3.5 give a good visual impression of the results, the fact that ground truth motion is available for the landmark points also offers the possibility to have a quantitative measure of the quality of the estimated motion field. Such a quantitative measure to assess and compare the performance of the method has been introduced with the so-called average angular error (AAE) in [8] and weighted angular error (WAE) in [115]. Assume the computed motion field $V_{c}=\left(u_{c}, v_{c}\right)$ and the ground truth vector field $V_{g}=\left(u_{g}, v_{g}\right)$, the AAE of the calculated flow field can be computed by: 

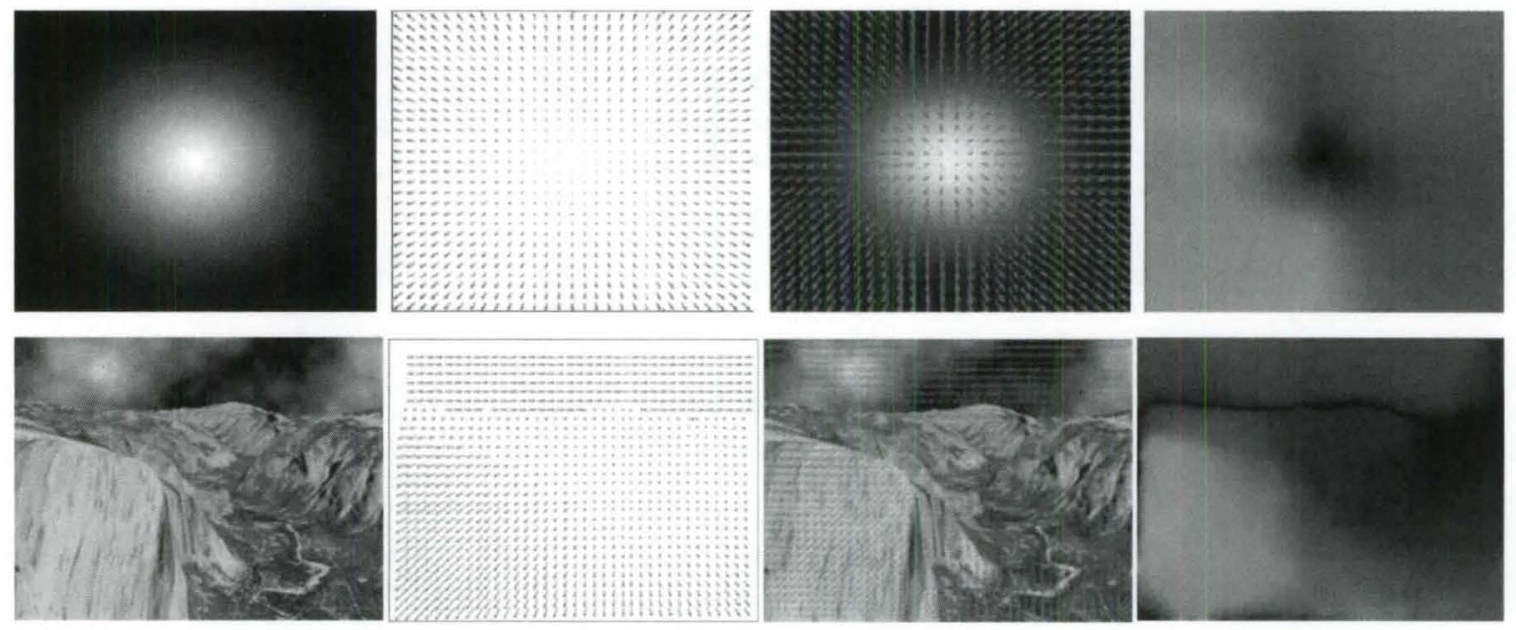

Figure 3.4: (top row) the image $I(x, y, t)=\exp \left(-(x-u t)^{2}-(y-v t)^{2}\right)$. (bottom row) Yosemite sequence with cloudy sky (316x252x15), frame 9. From Left To Right: (a) First frame. (b) Ground Truth. (c) Calculated vector field superimposed on the original image. (d) Color coded diagram of the velocity field (based on Figure 3.3).

$$
A A E:=\frac{1}{n} \sum_{i=1}^{n} \arccos \left(\frac{\left(u_{c}\right)_{i}\left(u_{g}\right)_{i}+\left(v_{c}\right)_{i}\left(v_{g}\right)_{i}+1}{\sqrt{\left(\left(u_{c}\right)_{i}{ }^{2}+\left(v_{c}\right)_{i}{ }^{2}+1\right)\left(\left(u_{g}\right)_{i}{ }^{2}+\left(v_{g}\right)_{i}{ }^{2}+1\right)}}\right)
$$

where $n$ denotes the total number of the landmark points. This expression not only measures the spatial angular error between the estimated flow vector and the correct vector, but also the differences in the magnitude of both vectors, since it evaluates the angular error of the spatio-temporal vector $\left(u_{c}, v_{c}, 1\right)$.

In the WAE, the individual angle deviations have been weighed by the magnitude of the landmark displacement vector; normalized by the sum of magnitude of all ground truth vectors. The reason for this is to emphasize angle deviation of points which have large displacements, and similarly to de-emphasize the angle deviation of points which have a smaller displacement:

$$
\frac{1}{\sum\left|V_{g}\right|} \sum\left(\left|V_{g}\right| \cdot \arccos \frac{V_{g} \cdot V_{c}}{\left|V_{g}\right|\left|V_{c}\right|}\right)
$$



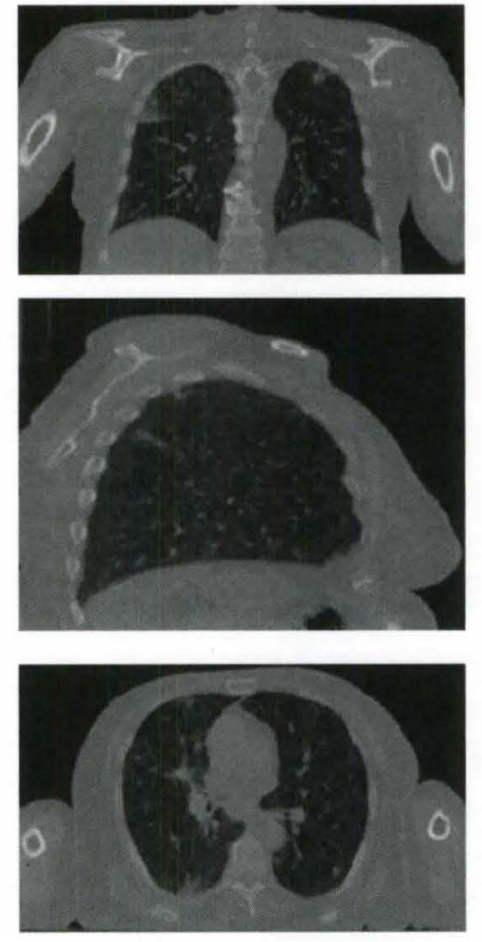
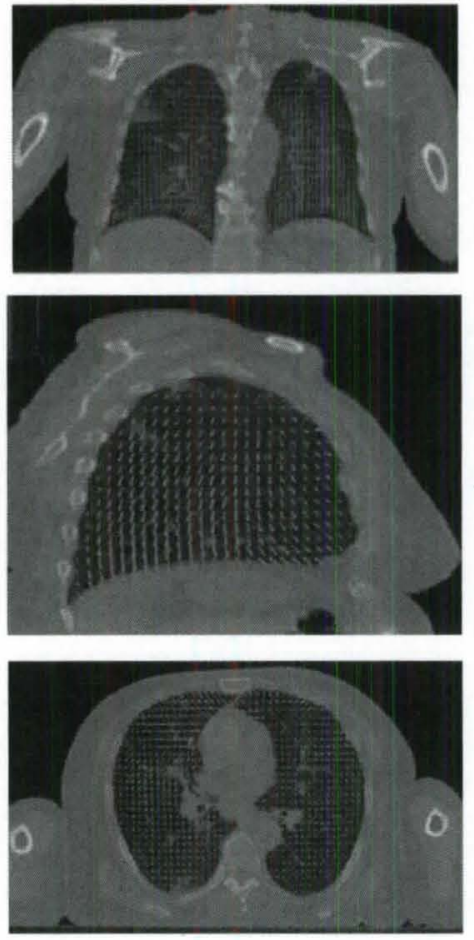
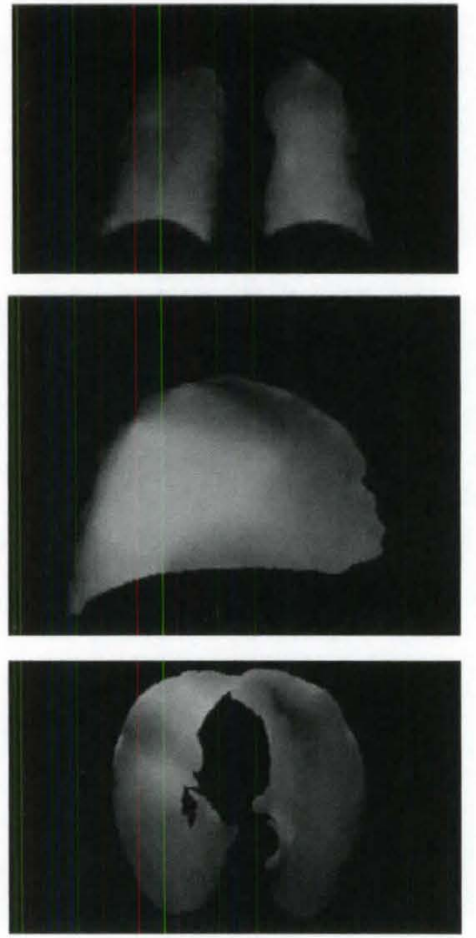

Figure 3.5: Top row: coronal, and bottom row: transversal planes of the lung. From left to right: (a) First frame. (b) Calculated vector field superimposed on the first frame with $\alpha=50, \gamma=150, \beta=1$. (c) Color coded diagram of the related vector filed (based on Figure 3.3).

Table 3.1 reports these results in comparison with the well-known optical flow methods which were reported in [115]. As shown in table 3.1, MOFID was superior in both AAE and WAE, meaning that amongst the optical flow algorithms which were evaluated, we should expect better estimation of the lung's planar motion using the proposed method.

\subsubsection{Three Dimensional Experiments}

For both qualitative and quantitative evaluation of MOFID, the publicly available POPI-model [173] and DIR-lab [19] data of lung deformation has been used. In the POPI-model dataset, 41 homologous landmarks were defined by experts in each of the ten respiratory phases of a single individual that make up this 4-D CT dataset with voxel dimensions $0.97 \times 0.97 \times 2 \mathrm{~mm}$ and made up of $512 \times 512 \times 141$ voxels. For the DIR-lab data, 75 landmarks for each time point between time phases P00 and P50 are available. DIR-lab 
Table 3.1: Quantitative evaluation and comparison of the already implemented methods and the proposed method, MOFID. AAE and WAE stand for average angular error and weighted angular error, respectively. Mean errors are shown with (standard deviation) for each case.

\begin{tabular}{ccc}
\hline Method & AAE & WAE \\
\hline Horn-Schunck (Global) [66] & $92.65^{\circ}\left(9.3^{\circ}\right)$ & $91.4^{\circ}\left(10.6^{\circ}\right)$ \\
\hline Lucas-Kanade (Local) [96] & $92.62^{\circ}\left(11.5^{\circ}\right)$ & $92.22^{\circ}\left(12.8^{\circ}\right)$ \\
\hline CLG (Local-Global) [16] & $89.88^{\circ}\left(9^{\circ}\right)$ & $89.7^{\circ}\left(9.8^{\circ}\right)$ \\
Brox et al. [14] & $28.93^{\circ}\left(3.7^{\circ}\right)$ & $36.1^{\circ}\left(3.9^{\circ}\right)$ \\
Wildes et al. [184] & $28.22^{\circ}\left(3.5^{\circ}\right)$ & $35.89^{\circ}\left(3.8^{\circ}\right)$ \\
MOFID & $26.98^{\circ}\left(3.1^{\circ}\right)$ & $34.2^{\circ}\left(3.2^{\circ}\right)$ \\
\hline
\end{tabular}

data has ten 4-D CT lung images from patients treated for thoracic malignancies. The 4-D image characteristics of the ten cases utilized in this study are given in Table 3.2. The accuracy of MOFID relative to the known expert-defined landmarks of both the POPI-model and DIR-lab data are reported here.

Two evaluation measures are employed for both POPI-model and DIR-lab data: the primary measure is the accuracy of the registration results and the secondary measure is physical plausibility of the computed deformation.

To measure the accuracy of the registration results, the computed transformation has applied to the ground truth point set from consecutive phases and compared estimated positions to the actual positions of the ground truth points. The differences between the estimated positions with the actual position are referred to as ground truth discrepancies or target registration error (TRE). The registration result is reported as $\bar{\mu}_{g t} \pm \sigma_{\mu_{g t}}$ where $\bar{\mu}_{g t}$ denotes the average of all ground truth discrepancies and $\sigma_{\mu_{g t}}$ is the standard deviation.

Figure 3.6 presents the calculated motion field of POPI-model dataset between two consecutive respiratory phases P30 and P40 in volumetric form. Adopting the NMI-based method to find the optimum weights, $\alpha, \gamma$, and $\beta$ were chosen to be 25,80 , and 1 , respectively and three scales were used in the multiscale strategy. 
Table 3.2: Spatial accuracy of the proposed algorithm, MOFID, for the 75 landmarks and six time points of DIR-lab data sets. Average spatial errors are reported in millimeters with standard deviation (SD) in parentheses. The fourth column exhibits the average landmark displacements before registration. The fifth column represents the errors subsequent to deformable registration.

\begin{tabular}{ccccc}
\hline Patient & Image dimension & Voxel dimension $(\mathbf{m m})$ & $\begin{array}{c}\text { Average Landmark } \\
\text { displacements }\end{array}$ & $\begin{array}{c}\text { Average } \\
\text { registration error }\end{array}$ \\
\hline Case 1 & $256 \times 256 \times 94$ & $(0.97 \times 0.97 \times 2.5)$ & $4.01(2.91)$ & $0.91(0.9)$ \\
\hline Case 2 & $256 \times 256 \times 112$ & $(1.16 \times 1.16 \times 2.5)$ & $4.65(4.09)$ & $1.08(1.06)$ \\
\hline Case 3 & $256 \times 256 \times 104$ & $(1.15 \times 1.15 \times 2.5)$ & $6.73(4.21)$ & $1.52(1.15)$ \\
\hline Case 4 & $256 \times 256 \times 99$ & $(1.13 \times 1.13 \times 2.5)$ & $9.42(4.81)$ & $1.91(1.39)$ \\
\hline Case 5 & $256 \times 256 \times 106$ & $(1.10 \times 1.10 \times 2.5)$ & $7.10(5.14)$ & $1.66(1.45)$ \\
\hline Case 6 & $512 \times 512 \times 128$ & $(0.97 \times 0.97 \times 2.5)$ & $11.10(6.98)$ & $2.05(1.75)$ \\
\hline Case 7 & $512 \times 512 \times 136$ & $(0.97 \times 0.97 \times 2.5)$ & $11.59(7.87)$ & $2.03(1.51)$ \\
\hline Case 8 & $512 \times 512 \times 128$ & $(0.97 \times 0.97 \times 2.5)$ & $15.16(9.11)$ & $2.5(1.85)$ \\
\hline Case 9 & $512 \times 512 \times 128$ & $(0.97 \times 0.97 \times 2.5)$ & $7.82(3.99)$ & $1.49(0.95)$ \\
\hline Case 10 & $512 \times 512 \times 120$ & $(0.97 \times 0.97 \times 2.5)$ & $7.63(6.54)$ & $1.71(1.38)$ \\
\hline
\end{tabular}

Considering all 9 registrations of the POPI-model, the average 3-D Euclidean distance between the computed and actual ground truth points with the proposed optical flow method was $1.02 \pm 0.70 \mathrm{~mm}$ $(3.3 \pm 2 \mathrm{~mm}$ was the mean landmark distance without registration). The average computational time to arrive at the motion field between two volumetric frames was 74 minutes on a Dell 5160 machine with a quad-core Xeon $3.00 \mathrm{GHz}$ processor. A similar qualitative evaluation of the method for the 75 landmarks in six time points of the DIR-lab data is summarized in Table 3.2. Similarly, adopting the method in II.B, $\alpha$, $\gamma$, and $\beta$ were chosen to be 10,110 , and 1 , respectively, and three scales were used in the multi-scale strategy.

The physical plausibility of the deformation field was measured using the determinant of the Jacobian of the deformation field which is directly related to specific volume changes [137]. If the Jacobian is unity, there is no local expansion or contraction. If the Jacobian is greater than one, there is local tissue 


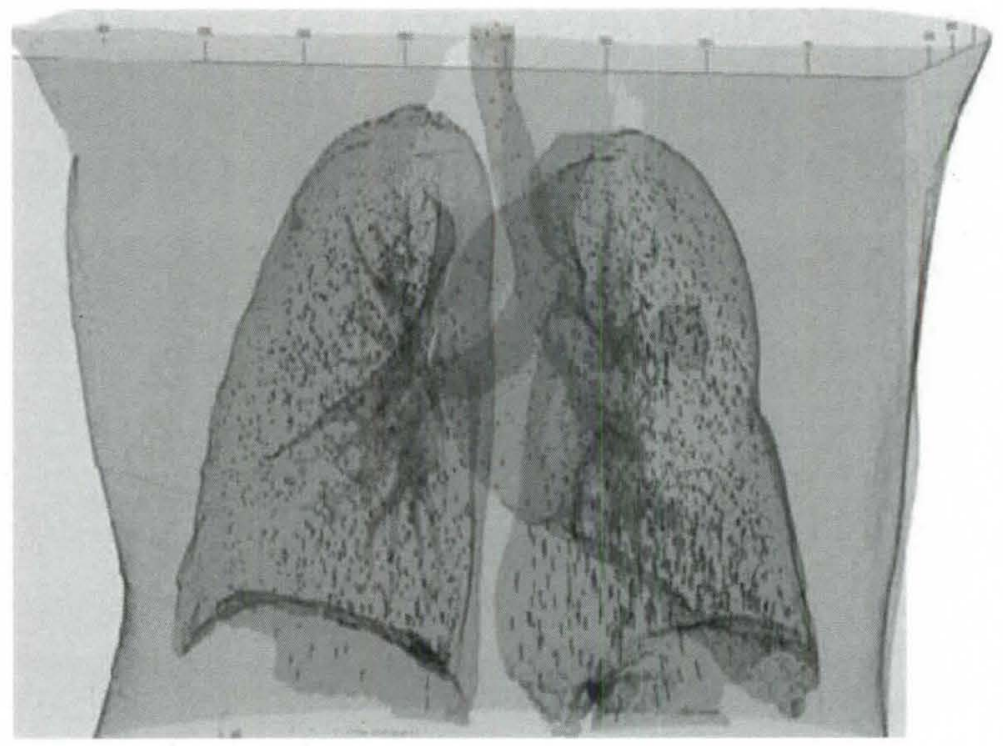

Figure 3.6: The computed volumetric motion field for the POPI-model dataset between two consecutive phases 30 and 40 .

expansion; if the Jacobian is less than one, there is local tissue contraction. In particular, we expect the calculated deformation field to be bijective. Regions where the deformation field is not bijective has a Jacobian determinant of zero and is a singularity [108]. Additionally, a negative Jacobian implies a deformation that is physically not permissible. Figure 3.7 shows box plot of the Jacobian value for each of the 9 calculated deformation maps between successive respiratory phases of the POPI-model data as well as the color-coded Jacobian between phases 30 and 40 for the mid-coronal imaging plane.

Figure 3.8 shows a box plot of the error range at each two successive respiratory phases for POPImodel data, using the cumulative set of all 41 annotated landmarks. A trend toward bigger magnitude errors between phases P20 and P30 at exhale as well as P60 and P70 at inhale is visually apparent, consistent with the correlation between landmark displacements between each two successive respiratory phases [173].

Figure 3.9 shows a similar box plot of the error range at each two successive respiratory phases from the end of inspiration to the end of expiration, using the cumulative set of all 75 annotated landmarks for all 10 DIR-lab data sets. As expected, a similar trend toward a bigger TRE for the respiratory phases with bigger displacement can be seen here. 


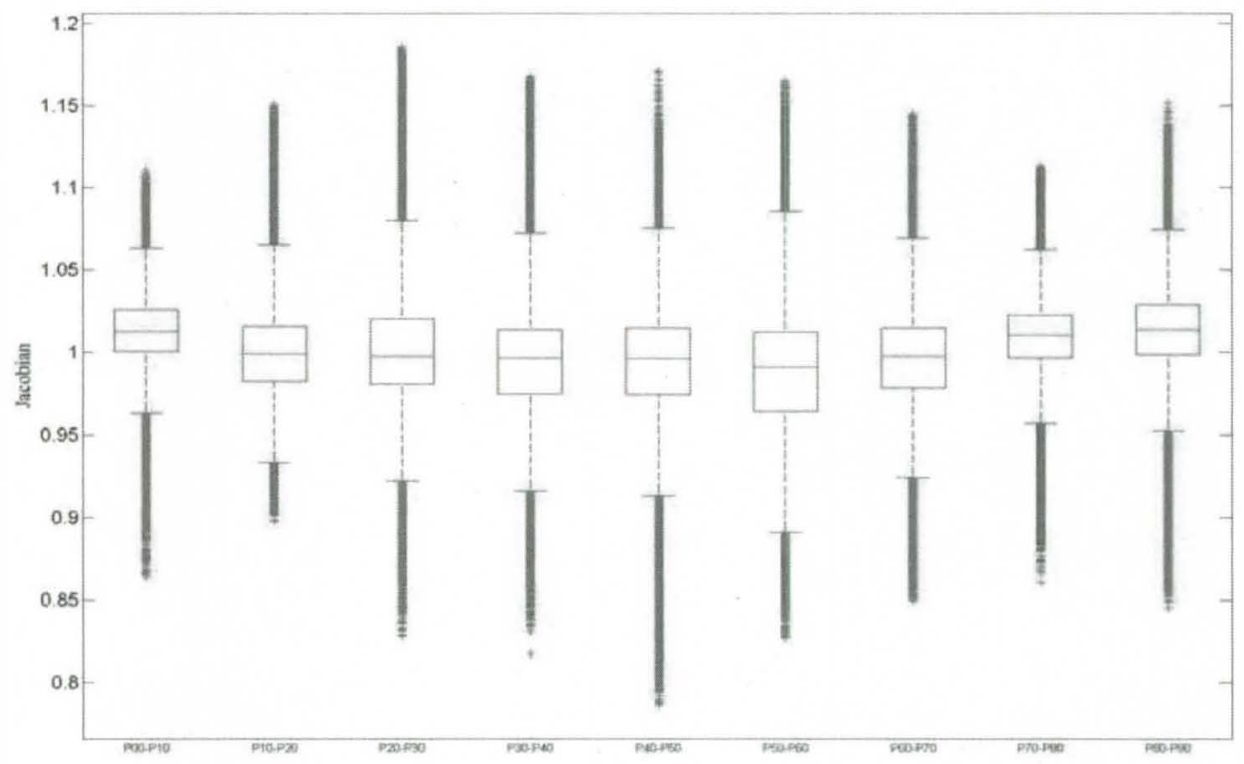

(a)

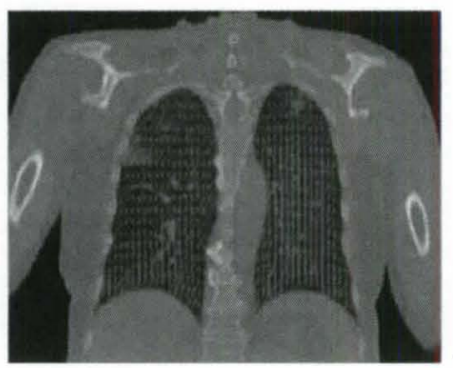

(b)

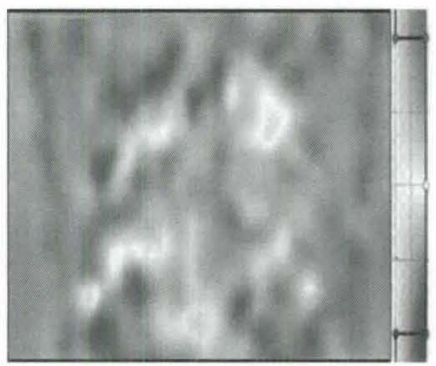

(c)

Figure 3.7: (a) The distribution of calculated Jacobian of lung for the computed deformation field between consecutive phases of the POPI-model data. For each box, the central mark is the median of all computed values, the edges of the box are the 25 th and 75 th percentiles, the whiskers extend to the most extreme data points not considered outliers, and outliers are plotted individually. (b) The coronal plane of the calculated motion field of the POPI-model in Figure 3.6 between phases P 30 and P40 volumetric frames and (c) the related Jacobian image.

The average resulting error of MOFID over the expert-defined homologous landmarks is found to be comparable to previously reported optical flow registration methods; as stated in Table 3.3, Castillo et al. [18] proposed a combined compressible local global (CCLG) method and achieved mean registration $1.02 \pm 1.03 \mathrm{~mm}, 1.29 \pm 1.22 \mathrm{~mm}$, and $2.50 \pm 1.91 \mathrm{~mm}$ on first, second, and fifth case of DIR-lab, 


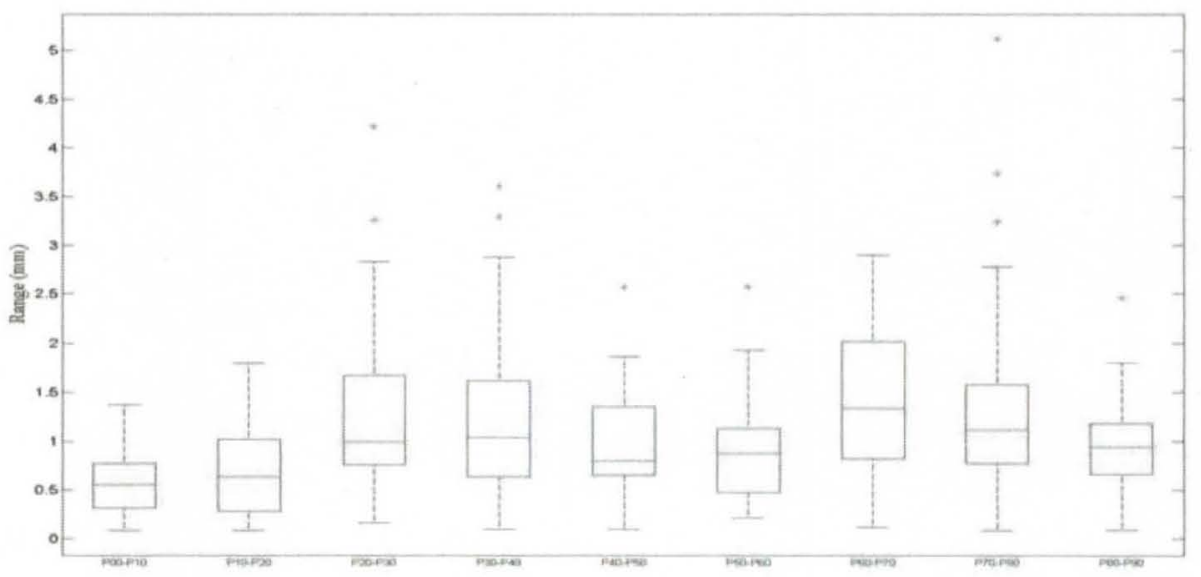

Figure 3.8: Phase-step target registration error (TRE) for POPI-model data. A box plot is shown illustrating the range of MOFID magnitude registration errors for each of the two consecutive respiratory phases of the data. The errors for the 41 annotated landmarks were pooled to produce the plot.

respectively, while the compressible optical flow method by Wildes [184] achieved $1.17 \pm 1.07$ $m m, 1.37 \pm 1.27 \mathrm{~mm}$, and $2.57 \pm 1.85 \mathrm{~mm}$ of error for same dataset. To the best of our knowledge, performance of CCLG and COF methods have not been reported on the remaining seven DIR-lab data sets. Our results which were determined from all 10 available DIR lab data sets improves on previously reported values and motivates the use of physical constraints which were utilized in our numerical implementation. The incorporated spatio-temporal regularization in the optical flow formulation forces the deformation to be continuous and differentiable in both the spatial and temporal domain in addition to helping to alleviate motion artifact inherent to dynamic images as well as avert singularities in Jacobian of the deformation.

It should be noted that the temporal smoothness need be regulated wisely to take into account the expected smoothness of the motion of the lung so as not to sacrifice the registration accuracy. Therefore, the optimum and effective incorporation of the aforementioned assumptions in the optical flow energy function is nontrivial. An NMI-based method has been proposed to find the optimum combination of weighting parameters. Figure 3.10 shows the sensitivity of TRE to different combination of $\alpha$ and $\gamma$ values which set the importance of the regularization and gradient terms. Sensitivity of TRE to $\alpha$ and $\gamma$ values has been evaluated for motion estimation between phases P30 and P40 which exhibits the largest deformation of all the exhale phases of the respiratory cycle (Figure 3.8). 

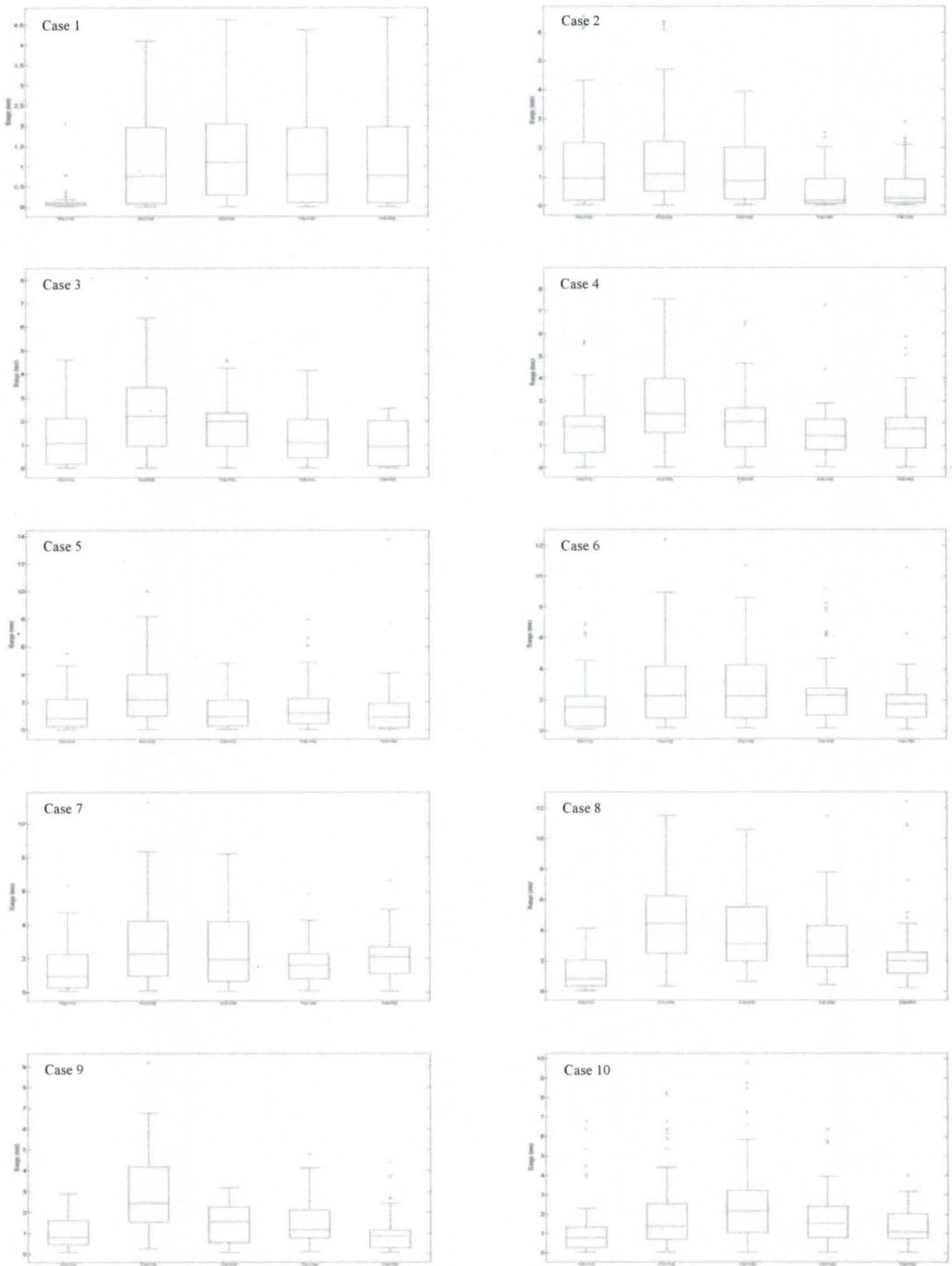

Figure 3.9: Phase-step target registration error (TRE) for DIR-lab data - please see Table 3.2. A box plot is shown illustrating the range of MOFID magnitude registration errors for each of the two consecutive respiratory phases for all of the DIR-lab data. For each of the 10 subjects, 75 annotated landmarks were combined to pool the measured errors. 
Table 3.3: Spatial accuracy of the proposed algorithm, MOFID, in comparison with compressible optical flow (COF) and compressible combined local global (CCLG) method.

\begin{tabular}{|c|c|c|c|c|}
\hline Patient & Image dimension & Voxel dimension $(\mathrm{mm})$ & $\begin{array}{l}\text { Average Landmark } \\
\text { displacements }\end{array}$ & $\begin{array}{c}\text { Average registration } \\
\text { error }\end{array}$ \\
\hline Case 1 & $256 \times 256 \times 94$ & $(0.97 \times 0.97 \times 2.5)$ & $4.01(2.91)$ & $\begin{array}{c}1.17(1.07) \text { COF } \\
1.02(1.03) \text { CCLG } \\
0.91(0.9) \text { MOFID }\end{array}$ \\
\hline Case 2 & $256 \times 256 \times 112$ & $(1.16 \times 1.16 \times 2.5)$ & $4.65(4.09)$ & $\begin{array}{c}1.37(1.27) \text { COF } \\
1.29(1.22) \text { CCLG } \\
\mathbf{1 . 0 8}(\mathbf{1 . 0 6 )} \text { MOFID }\end{array}$ \\
\hline Case 5 & $256 \times 256 \times 106$ & $(1.10 \times 1.10 \times 2.5)$ & $7.10(5.14)$ & $\begin{array}{c}2.57(1.85) \text { COF } \\
2.50(1.91) \text { CCLG } \\
\mathbf{1 . 6 6}(\mathbf{1 . 4 5 )} \text { MOFID }\end{array}$ \\
\hline
\end{tabular}

Based on Figure 3.10 and as expected, changing $\alpha$ has a significant impact on TRE, most likely because of the resulting increase in effectiveness of the regularization term in the energy function. For $\alpha$ values less than 10 , the regularization term is not effective enough and TRE starts to grow. $\alpha=10$ has been a good choice in terms of the mean and error range of TRE. On the other hand, specific values for $\gamma$ (shown here with different colors) plays a highly significant role on TRE value. Indeed, turning off the gradient constancy term by setting $\gamma=0$ leads to the largest value for TRE.

We have also evaluated the convergence behavior of MOFID which iteratively arrives at the final solution based on the SOR method [133]. Figure 3.11 plots the normalized difference between the value of the energy function at two successive iterations for three scales. 


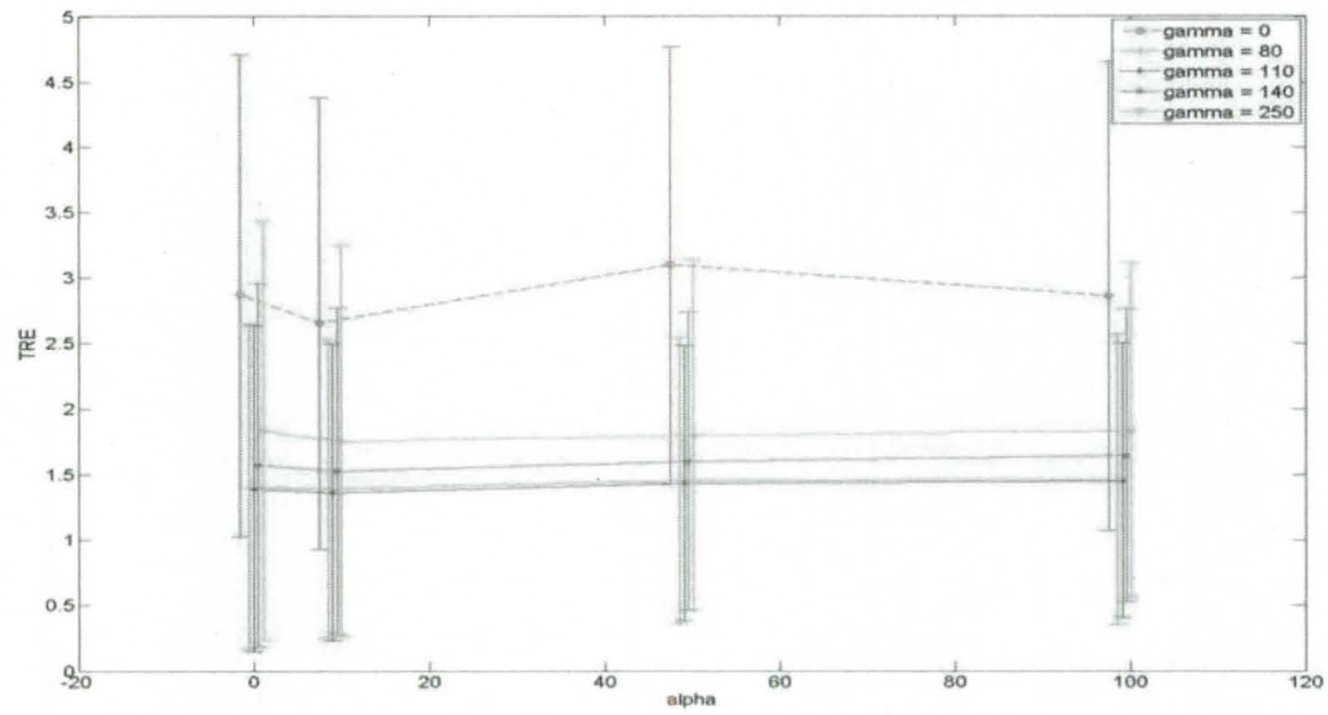

Figure 3.10: Sensitivity of total registration error (TRE) to $\alpha$ value and $\gamma$ value. These error bars show the mean and error range of TRE in mm in terms of four different $\alpha$ values, $1,10,50$, and 100 and five different $\gamma$ values, 0 (red), 80 (green), 110 (blue), 140 (magenta), and 250 (cyan) over all 41 annotated landmarks for POPI-model between phases P30 and P40 volumetric frames. 


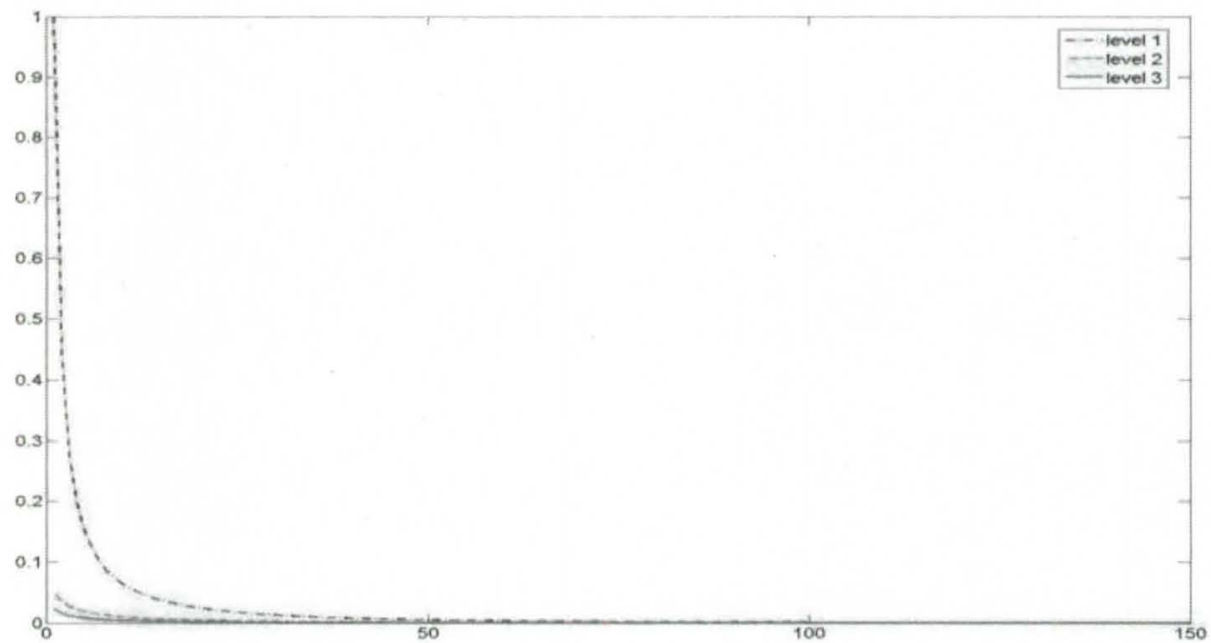

Figure 3.11: The normalized difference between two successive calculated energy function values in terms of SOR iterations $\left(\frac{\left\|E_{i+1}-E_{i}\right\|}{\left\|E_{i}\right\|}\right)$ for motion estimation between phases P30 and P40 of the POPImodel data at three scales. 


\section{CHAPTER 4}

\section{LANDMARK-BASED ELASTIC REGISTRATION FOR ESTIMATING LARGE DEFORMATIONS}

\subsection{Introduction}

The success of most PDE based DIR methods depends in a important way on the accuracy of finite difference approximations to image derivatives. As a result, factors such as image artifact and large voxel displacements affect this accuracy and can lead to significant registration errors. For example, breath-hold thoracic CT images (Figure 4.1) which are acquired at opposite ends of the breath cycle, follow-up CT images (Figure 4.2), or images from different scaners with the possibility of the different modalities all falling, within the scope of this study, depict large voxel displacements. In particular, follow-up studies of lung cancer patients entail large deformation IR due to change in lung/tumor definition.

An important problem in radiation treatment planning is to determine how a target volume changes during the course of a breathing cycle or during the course of treatment. Clearly, physiological movement of the patient or organs affects the definition of the target volume [146, 162]. A second challenge arises from the need to register the post-treatment lung with the pre-treatment lung in order to evaluate the effectiveness of the treatment and in order to correlate injury to the normal lung with the radiation dose received. This is difficult not only because the tumor volume changes but because normal lung is also affected by radiation treatment as it becomes fibrotic leading to completely different Hounsfield units posttreatment. The proposed optical flow method in Chapter 3, like most PDE-based methods, is not capable of handling large voxel displacements. One consideration that can prove useful in the design of algorithms for this problem is that bifurcations of bronchi provide reliable features which may be utilized as part of landmark-based deformable image registration.

To achieve the goal of landmark-based image registration, automatic landmark localization is important, as manual selection of landmarks is time-consuming and often inaccurate. In addition to land- 

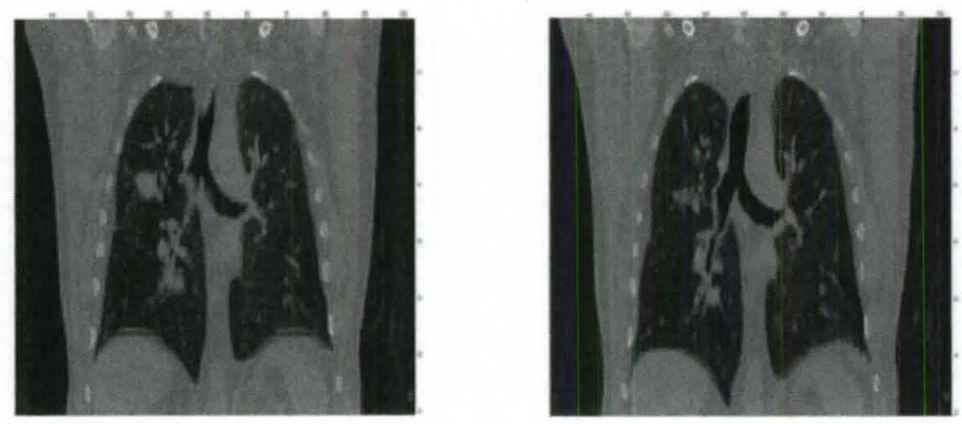

Figure 4.1: A pair of corresponding slices from 4-D CT data at end-exhale (left) and end-inhale (right) respiratory phases.

mark based registration, which uses local feature information, a B-spline transformation model [69, 87, $135,144,166]$ guided by mutual information similarity metric $[22,68,157]$ is also proposed here to guarantee the smoothness of transformation. The proposed method therefore uses a hybrid local/global approach to deformable registration with landmarks providing the local information and the mutual information-based registration providing the global statistical intensity information. Since during normal breathing, the lung undergoes large nonlinear deformations, it is expected that the proposed method would also be applicable to large deformation registration between maximum inhale and maximum exhale images in the same subject. The proposed framework can be used to co-locate SPECT and CT volumes, though with a different choise of landmarks (expanded upon in Chapter 6). As described in this chapter, the method has been evaluated by registering 3-D CT volumes at maximum exhale data with all the other temporal volumes in the POPI-model data [125].

\subsection{Path Planning and Landmark Extraction}

In order to register the pre-treatment volume to post-treatment volume, there is a need to find robust and homologous features which are not affected by the radiation treatment along with a smooth deformation field. Since airways are well-distributed in the entire lung, we propose use of airway tree bifurcations for registration of the pre-treatment volume to the post-treatment volume. Estimation of lung deformation via matching of corresponding landmarks is possible because of the identification of airway and the bifurcations. 

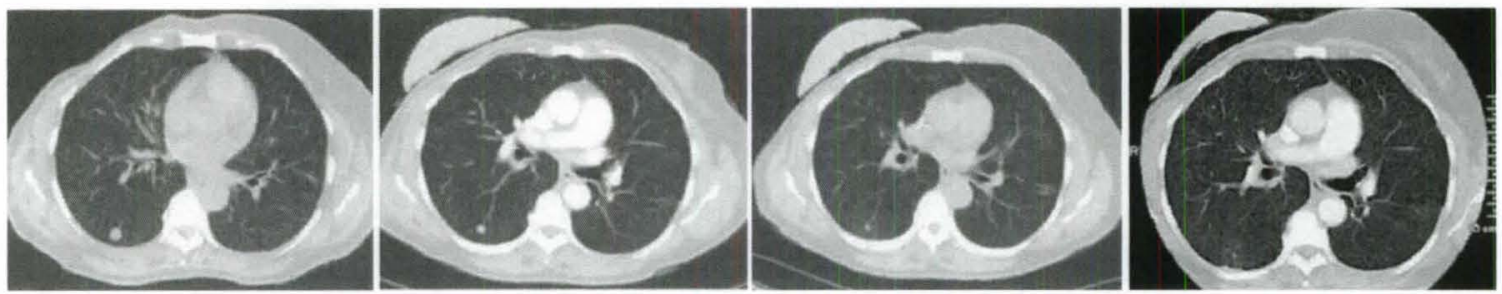

Figure 4.2: CT scans showing tumor response and treatment accuracy of TomoTherapy SBRT. From left to right: (a) pre-treatment, (b) two months after treatment, (c) three months after treatment, (d) six months after treatment [Courtesy of University of Virginia].

Landmarks were extracted from the segmented airway tree of the lung. For airway tree segmentation a level-set segmentation with region competition with ITK-SNAP was used [200]. Subsequently, the path planning algorithm by Negahdar et al. [112-114] was used. The output of the latter algorithm is a centerline representation of the segmented airways tree- it automatically produces all of the end points along with the labeled bifurcation point. Figure 4.3 illustrates the output of the path planning method. The bifurcation landmark points consist of three airway branches with radii $r_{i}, i=1,2,3, \frac{1}{m} \sum r_{i}>\bar{R}-\sigma_{R}$ where $\bar{R}$ and $\sigma_{R}$ denote the average and standard deviation of all radii in the centerlines of the whole airways tree, this condition removes small branches that are likely to vanish between the CT slices. To filter those bifurcations where one branch significantly differs in its radius from the other two, a MAD-based outlierrejection method has been adopted; $\forall r_{i}: \frac{\left|r_{i}-\operatorname{median}\left(r_{i}\right)\right|}{\operatorname{MAD}\left(r_{i}\right)}<2$ where $M A D\left(r_{i}\right)$ is the median absolute deviation of the radii of branches at that particular bifurcation. Landmark based registration has the advantage of matching landmarks either exactly or inexactly based on the confidence in the specific landmark location.

In general, the landmark image registration problem can be thought of as a Dirichlet problem [74, 75] and can be stated mathematically as finding the displacement field $u$ that minimizes the cost function:

$$
\mathcal{C}_{L M}=\int_{\Omega}\|\mathcal{L} u(x)\|^{2} d x
$$

subject to the constraint that $u\left(p_{i}\right)=q_{i}-p_{i}$ over all bifurcations used as landmarks. The operator $\mathcal{L}$ denotes a symmetric linear differential operator and is used to interpolate $u$ away from the corresponding landmarks $[6,12]$. 

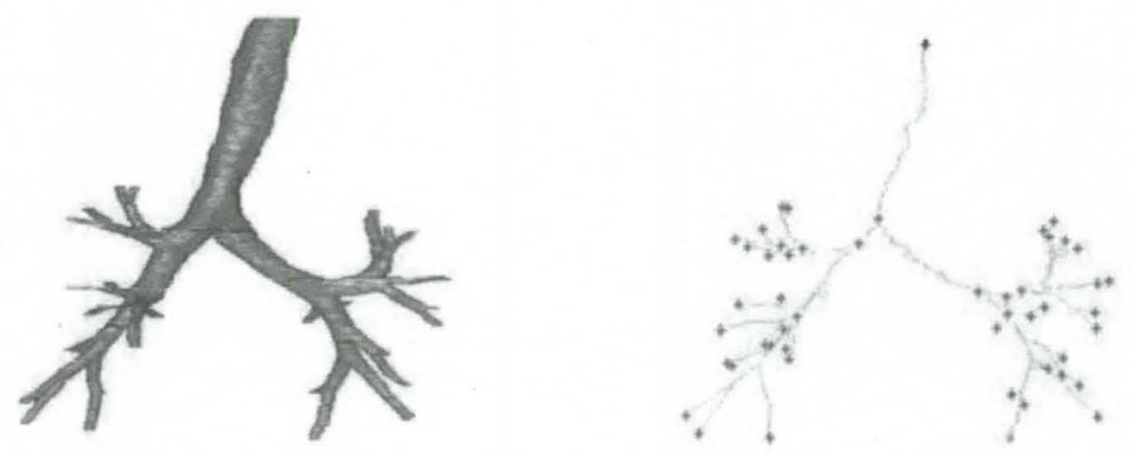

Figure 4.3: (Left) segmented airways. (Right) the extracted landmark points with the proposed path planning method.

\subsection{Transformation Estimation}

Having identified the corresponding landmarks we estimate a volumetric transformation $\mathcal{T}$ that maps the post-treatment landmark set $\left\{q_{i}\right\}$ onto the pre-treatment landmark set $\left\{p_{i}\right\}$. A B-spline transformation model guided by mutual information similarity metric is used because the compact support of B-splines keeps the running time reasonably low for volumetric and time series image dataset

$$
\mathcal{T}_{\mu}(x)=x+\sum_{x_{k} \in \mathcal{N}_{x}} p_{k} \beta^{3}\left(\frac{x-x_{k}}{\sigma}\right)
$$

with $x_{k}$ being the control points, $\beta^{3}(x)$ the cubic B-spline polynomial, $p_{k}$ the B-spline coefficient vectors, $\sigma$ the B-spline control point spacing, and $\mathcal{N}_{x}$ the set of all control points within the compact support of the B-spline at $x$. B-spline in comparison with other spline-based transformation models, such as thin-plate spline $[12,63,143]$ or elastic-body spline $[29,186]$, is locally controlled. One of the main motivations behind the use of B-spline transformation is the implicit regularization embedded in the B-spline basis functions which guarantees the smoothness of transformation. The cost function $\mathcal{C}_{M I}$ guided by mutual information similarity metric is minimized with respect to $\mathcal{T}$. The total cost function $\mathcal{C}_{T}=\lambda \mathcal{C}_{L M}+\mathcal{C}_{M I}$ is optimized using the gradient descent method.

\subsection{Experimental Results}

The deformation map between the remaining 9 phases of the publicly available point-validated pixelbased POPI model and the end-exhale image of the same dataset which is used as the reference is 

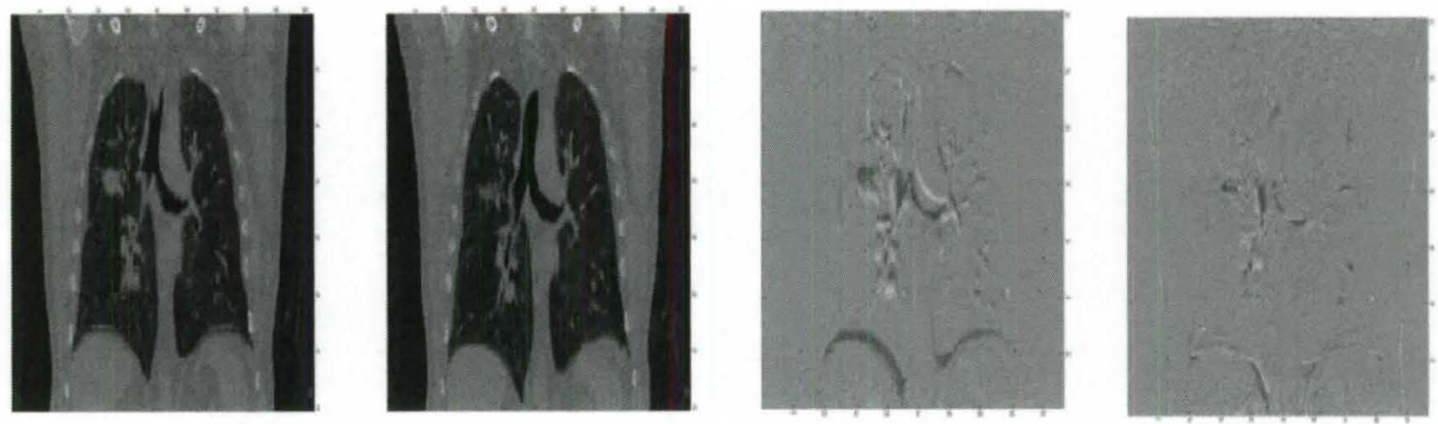

Figure 4.4: (a) Reference image at end-exhale, (b) deformed image at inhale. Difference image (c) before registration and (d) after application of the MI-B-spline and landmark-based registration.

calculated. In the POPI model data, 41 homologous landmarks were defined by experts in each of the 3-D volumes that make up this 4-D CT data set with voxel dimensions $0.97 \times 0.97 \times 2 \mathrm{~mm}$ [173]. The accuracy of the proposed method relative to the known expert-defined landmarks of the POPI-model is reported.

The estimated transformation is applied to the ground truth point set from different phases and compared estimated positions in the maximum exhale phase to the actual positions of the ground truth points. The differences between the estimated positions with the actual positions are referred to as ground truth discrepancies. The registration result is reported as $\bar{\mu}_{g t} \pm \sigma_{\mu_{g t}}$ where $\bar{\mu}_{g t}$ denotes the average of all ground truth discrepancies and $\sigma_{\mu g t}$ is the standard deviation. The average Euclidean distance between the ground truth points was reduced from $1.85 \pm 1.43 \mathrm{~mm}$ for deformable B-spline registration to $1.33 \pm 0.99$ $\mathrm{mm}$ by having the bifurcation landmarks. Referring to other landmark-based lung registration methods, Urschler et al. [171] used point correspondence from lung and diaphragm surface for a thin-plate spline registration and achieved mean registration error in the range of 5 to $9 \mathrm{~mm}$ in experiments on CT sheep scans with voxel dimensions of $0.5 \times 0.52 \times 0.6 \mathrm{~mm}$. Coselmon et al. [27] applied gray value based image registration using mutual information and thin-plate spline interpolation. They achieved an alignment accuracy of $1.7 \mathrm{~mm}, 3.1 \mathrm{~mm}, 3.6 \mathrm{~mm}$ in the left-right, anterior-posterior, inferior-superior directions. Hilsmann et al. [63] used vessel bifurcations from lung for a thin-plate spline registration and achieved mean registration error $2.85 \pm 2.11 \mathrm{~mm}$ for thin-plate spline and $3.40 \pm 2.38 \mathrm{~mm}$ for affine registration on 


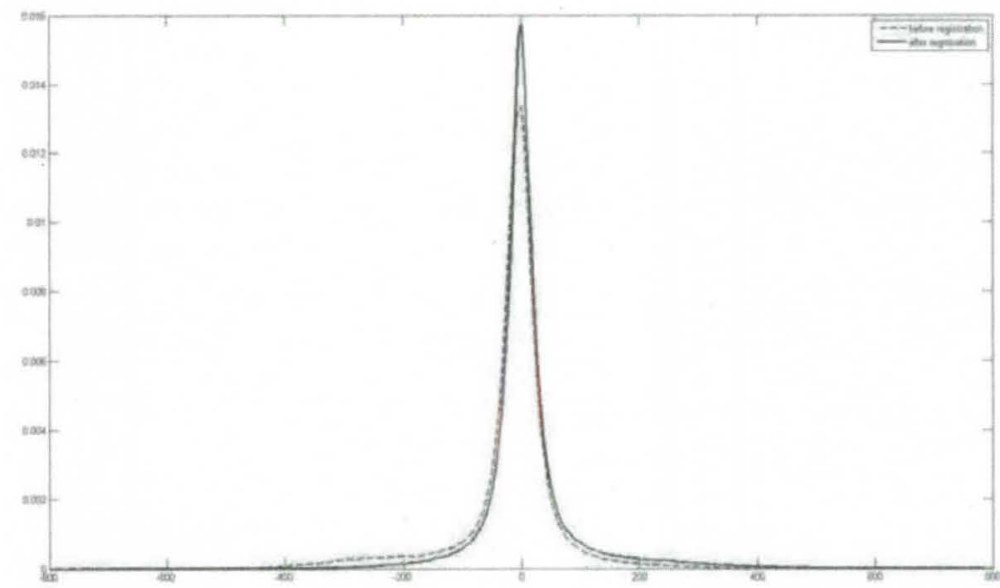

Figure 4.5: Histogram of difference images before (red dashed line) and after (blue solid line) registration. The histograms are normalized and weighed with the bin volume.

CT images with voxel size of $0.98 \times 0.98 \times 2.5 \mathrm{~mm}$. Compared to these registration results, we have been able to obtain smaller errors.

The second validation metric relies on the difference image before and after registration. We register the maximum inhale image to the maximum exhale image by applying the estimated transformation. We obtain a transformed version of the inhale image that, in the ideal case, is equal to the exhale image. Comparing the difference images before and after registration provides us with information about the registration result that depends on the image information only. Figure 4.4 presents an example of difference image in the coronal plane. As may be gathered from examining the difference image, the registration errors are reduced near the airways and near the diaphragm. In order to evaluate the difference images quantitatively, we build histograms of the difference images in the region of the lung. Figure 4.5 depicts difference image histograms before and after registration. According to Figure 4.5, after registration the number of points with values less than -200 in the difference image can be reduced significantly in contrast to the difference image before registration. Additionally, the number of points with value 0 , i.e. the number of points that have the same value in both images, increases after registration. Generally, the histogram changes in such a way that the left tail of the histogram drops off more quickly and the peak rises in exchange, signifying smaller errors after registration. 
The average computational time to arrive at these results was 44 minutes on a Dell Q6600 machine with a quad-core $2.4 \mathrm{GHz}$ processor and $3 \mathrm{~GB}$ memory.

The proposed parametric, elastic, free-form, landmark-based registration technique with automated landmark bifurcation extraction which was developed in this chapter, has the capability to assess breathing motion as well as follow-up studies of lung cancer patients involving large deformations with different lung/tumor definitions. Airways' bifurcation points have been chosen as robust landmarks to align the deformed lung over treatment or respiration. Landmark information improves the average registration errors when compared to elastic registration alone 


\section{CHAPTER 5}

\section{MEASURES OF REGIONAL MECHANICS FROM DEFORMATION MAPS}

\subsection{Introduction}

Many disease or injury conditions can cause biomechanical or structural property changes that can alter lung function. While the mechanical changes associated with the change of the material properties originate at a regional level, they are largely asymptomatic and invisible to global measures of lung function until they have advanced significantly and have aggregated. In fact, virtually all pulmonary diseases of either the obstructive or restrictive classification involve some abnormality of lung tissue mechanics [159].

Imaging has long been used to study lung mechanics. Some investigators have studied the linkage between estimates of regional lung expansion and local lung ventilation as a measure of pulmonary function $[20,21,35,56,57,124,137,160,162,190]$. We hypothesize that by determining regional and global lung elasticity and function, radiation treatment planning may be tailored to the individual patient.

This function information may be used to achieve conformal-avoidance radiation treatment planning. As a result, pulmonary toxicity may be more accurately predicted and prevented [33]. It would be desirable therefore to have objective methods with which to evaluate and follow the progression of disease based on measures of regional mechanics.

In this chapter, we present a novel approach to quantification and visualization of regional mechanical strain in the lung from 4-D CT using a multi-scale lung-specific optical flow deformable image registration method (MOFID) which was discussed in detail in Chapter 3. We propose to validate our method using a synthetic dataset and to apply it on the POPI-model data [122]. 


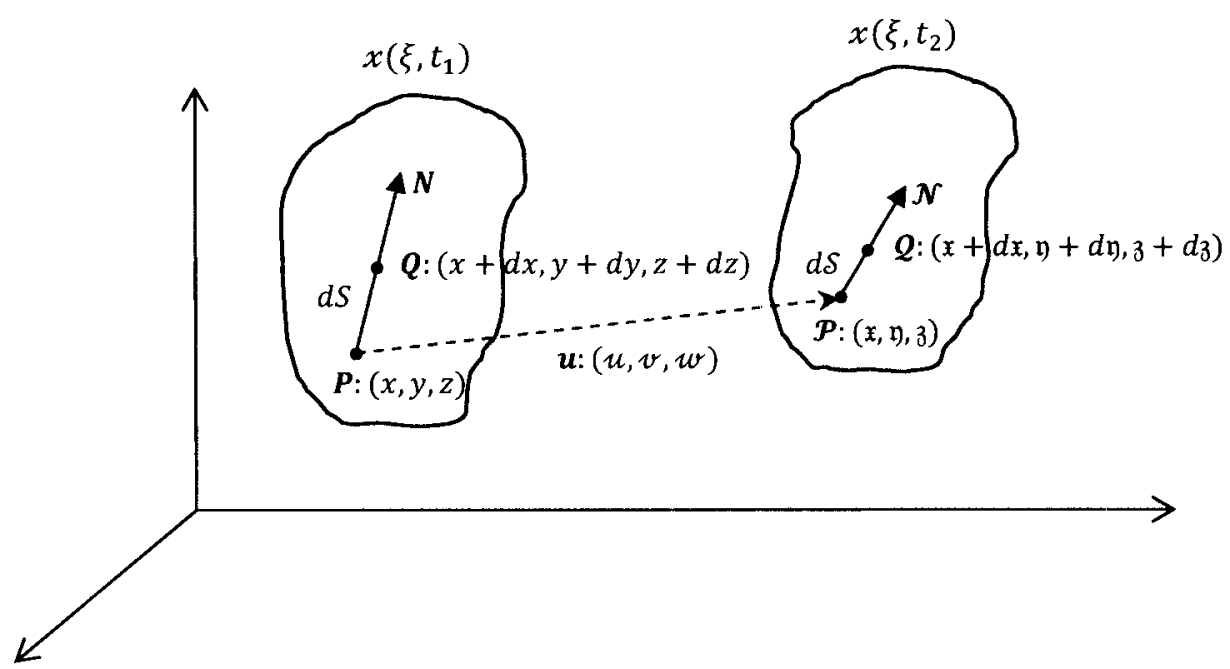

Figure 5.1: Deformation of a continuum body from the reference configuration, Lagrangian (left) to the current configuration, Eulerian (right) and elongation/compression of an infinitesimal line element under deformation.

\subsection{Regional Measures of Mechanics from Image Registration}

Figure 5.1 depicts a continuum body from the reference configuration at time $t_{1}$ that is deformed to the current configuration at time $t_{2}$. For a continuous bijective deformation $x\left(\xi, t_{1}\right) \rightarrow x\left(\xi, t_{2}\right)$ the line element $\boldsymbol{P Q}$ in region transforms to the line element $\mathcal{P} \mathcal{Q}[165]$.

In large deformation theory the elongation or compression of a line element is often defined as the normal strain. Strain is a dimensionless quantity representing the ratio change in length of a continuously deformable body. The length of the line element $\boldsymbol{P Q}$ is:

$$
d S^{2}=d x^{2}+d y^{2}+d z^{2}
$$

whereas the length of the line element $\mathcal{P Q}$ is:

$$
d \mathcal{S}^{2}=d \mathfrak{x}^{2}+d \mathfrak{y}^{2}+d \mathfrak{z}^{2}
$$

The expressions for the total differentials $\left(d x, d \mathfrak{y}, d_{3}\right)$ can be written in terms of $(d x, d y, d z)$ :

$$
\begin{aligned}
& d \mathfrak{x}=\frac{\partial x}{\partial x} d x+\frac{\partial x}{\partial y} d y+\frac{\partial x}{\partial z} d z \\
& d \mathfrak{y}=\frac{\partial \mathfrak{y}}{\partial x} d x+\frac{\partial \mathfrak{y}}{\partial y} d y+\frac{\partial \mathfrak{y}}{\partial z} d z \\
& d_{3}=\frac{\partial \mathfrak{z}}{\partial x} d x+\frac{\partial \mathfrak{y}}{\partial y} d y+\frac{\partial z}{\partial z} d z
\end{aligned}
$$


In addition, the displacement field $u:(u, v, w)$ relates the coordinates such that:

$$
u=\mathfrak{x}-x, \quad v=\mathfrak{y}-y, \quad w=\mathfrak{z}-z
$$

therefore, the previously written differential relationships in Equation (5-3) can be rewritten as:

$$
\begin{aligned}
& d x=\left(1+\frac{\partial u}{\partial x}\right) d x+\frac{\partial u}{\partial y} d y+\frac{\partial u}{\partial z} d z \\
& d \mathfrak{y}=\frac{\partial v}{\partial x} d x+\left(1+\frac{\partial v}{\partial y}\right) d y+\frac{\partial v}{\partial z} d z \\
& d_{z}=\frac{\partial w}{\partial x} d x+\frac{\partial w}{\partial y} d y+\left(1+\frac{\partial w}{\partial z}\right) d z
\end{aligned}
$$

therefore by Equation (5-1), (5-2), and (5-5) we obtain:

$$
\frac{1}{2}\left[d S^{2}-d S^{2}\right]=\epsilon_{11} d x^{2}+\epsilon_{22} d y^{2}+\epsilon_{33} d z^{2}+2 \epsilon_{12} d x d y+2 \epsilon_{13} d x d z+2 \epsilon_{23} d y d z
$$

where the coefficients are given by:

$$
\begin{aligned}
\epsilon_{11} & =u_{x}+\frac{1}{2}\left(u_{x}^{2}+v_{x}^{2}+w_{x}^{2}\right) \\
\epsilon_{22} & =v_{y}+\frac{1}{2}\left(u_{y}^{2}+v_{y}^{2}+w_{y}^{2}\right) \\
\epsilon_{33} & =w_{z}+\frac{1}{2}\left(u_{z}^{2}+v_{z}^{2}+w_{z}^{2}\right) \\
2 \epsilon_{12}=2 \epsilon_{21} & =v_{x}+u_{y}+u_{x} u_{y}+v_{x} v_{y}+w_{x} w_{y} \\
2 \epsilon_{13}=2 \epsilon_{31} & =w_{x}+u_{z}+u_{x} u_{z}+v_{x} v_{z}+w_{x} w_{z} \\
2 \epsilon_{23}=2 \epsilon_{32} & =w_{y}+v_{z}+u_{y} u_{z}+v_{y} v_{z}+w_{y} w_{z}
\end{aligned}
$$

If the Equation (5-6) is divided by $d S^{2}$ the normal strain for the line element $P \boldsymbol{Q}$ is obtained:

$$
\varepsilon=\frac{1}{2}\left[\left(\frac{d S}{d s}\right)^{2}-1\right]=\epsilon_{11} N_{1}^{2}+\epsilon_{22} N_{2}^{2}+\epsilon_{33} N_{3}^{2}+2 \epsilon_{12} N_{1} N_{2}+2 \epsilon_{13} N_{1} N_{3}+2 \epsilon_{23} N_{2} N_{3}
$$

where $N:\left(N_{1}, N_{2}, N_{3}\right)$ are the direction cosines corresponding to the reference configuration. Therefore, the normal strain of a line element implicitly contains information regarding the ratio of lengths of an infinitesimal line element before and after a continuous deformation. the Equation (5-8) can be rewritten in the tensorial formulation:

$$
\varepsilon=\left[\begin{array}{lll}
N_{1} & N_{2} & N_{3}
\end{array}\right]\left[\begin{array}{lll}
\epsilon_{11} & \epsilon_{12} & \epsilon_{13} \\
\epsilon_{21} & \epsilon_{22} & \epsilon_{23} \\
\epsilon_{31} & \epsilon_{32} & \epsilon_{33}
\end{array}\right]\left[\begin{array}{c}
N_{1} \\
N_{2} \\
N_{3}
\end{array}\right]=\boldsymbol{N}^{T} \boldsymbol{E} \boldsymbol{N}
$$

where $E$ is defined to be the Lagrangian strain tensor.

The Lagrangian strain tensor can also be derived directly from the relationship between the infinitesimal line element in the undeformed state, given by $\boldsymbol{d x}$ and its mapping to the deformed state, denoted by $\boldsymbol{d x}$ : 


$$
d x=F d x
$$

the deformation gradient tensor (DGT), $\boldsymbol{F}$, maps infinitesimal lengths between the undeformed and deformed state of a continuously deformable body. The elements of the DGT are:

$$
\boldsymbol{F}=\left[\begin{array}{lll}
\frac{\partial x}{\partial x} & \frac{\partial x}{\partial y} & \frac{\partial x}{\partial z} \\
\frac{\partial y}{\partial x} & \frac{\partial y}{\partial y} & \frac{\partial y}{\partial z} \\
\frac{\partial z}{\partial x} & \frac{\partial z}{\partial y} & \frac{\partial z}{\partial z}
\end{array}\right]
$$

in terms of the displacement field, $u:(u, v, w)$, the elements of the DGT are computed to be:

$$
\boldsymbol{F}=\left[\begin{array}{ccc}
1+u_{x} & u_{y} & u_{z} \\
v_{x} & 1+v_{y} & v_{z} \\
w_{x} & w_{y} & 1+w_{z}
\end{array}\right]
$$

Various kinematic descriptors of deformation can be calculated from the DGT. Because of the need for one-to-one correspondence between material points during continuous deformation, the determinant of the DGT, normally referred to as the Jacobian, has to be nonzero:

$$
J=\operatorname{det}(\boldsymbol{F})
$$

in addition, in the absence of any deformation the Jacobian is equal to unity. Since the deformation is assumed to be a continuous function of time, it is impossible for the Jacobian to be negative without passing through zero which was previously excluded from physical possibility. It therefore follows that for a continuous deformation to be physically possible, the Jacobian must be greater than zero. We took advantage of this fact to evaluate the accuracy of the calculated motion field which discussed in details in Section 2.4. The degree of regional lung expansion is measured using the Jacobian of the displacement field which is directly related to the specific volume change [137]. If the Jacobian is unity, there is no local expansion or contraction. If the Jacobian is greater than one, there is local tissue expansion; if the Jacobian is less than one, there is local tissue contraction.

DGT can be decomposed into stretch and rotation components:

$$
\boldsymbol{F}=\boldsymbol{R} \boldsymbol{U}
$$

where the $\boldsymbol{U}$ is the right stretch tensor and $\boldsymbol{R}$ is an orthogonal rotation tensor. The Cauchy-Green deformation tensor is defined as:

$$
\boldsymbol{C}=\boldsymbol{F}^{T} \boldsymbol{F}=\boldsymbol{U}^{2}
$$




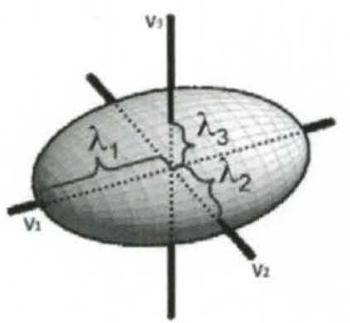

(a)

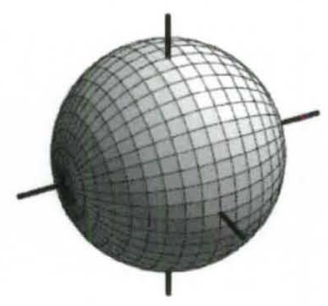

(b)

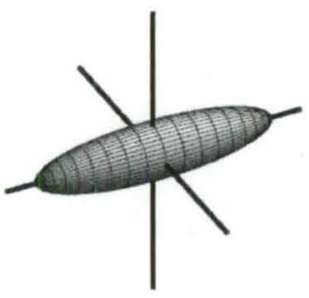

(c)

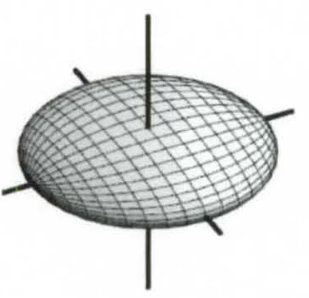

(d)

Figure 5.2: (a) Space of tensor shape. Three basic diffusion tensor shapes: (b) Isotropy, (c) Linear anisotropy, (d) Planar anisotropy.

The concept of strain may be used to evaluate how much a given displacement differs locally from a rigid body displacement. The Lagrangian strain tensor, $\boldsymbol{E}$, is then given by:

$$
\boldsymbol{E}=\frac{1}{2}(\boldsymbol{C}-\boldsymbol{I})=\frac{1}{2}\left(\boldsymbol{F}^{T} \boldsymbol{F}-\boldsymbol{I}\right)
$$

where $\mathbf{I}$ is the identity matrix. From the Lagrangian strain tensor we can calculate the various normal, shear, and principal strain values which describe the regional deformation of the element. Since $\boldsymbol{F}$ is non singular, the reverse mapping, $\boldsymbol{F}^{-1}$, exists and allows for the Eulerian description of strain, $\boldsymbol{G}$, which is given by:

$$
\boldsymbol{G}=\frac{1}{2}\left(\boldsymbol{I}-\left(\boldsymbol{F} \boldsymbol{F}^{T}\right)^{-1}\right)
$$

Eigenvalue decomposition on the Lagrangian strain tensor yields the three principal strain values, $\left(\lambda_{1}, \lambda_{2}, \lambda_{3}\right)$. The principal strain vectors, $\left(V_{1}, V_{2}, V_{3}\right)$, are the corresponding eigenvectors and correspond to the directions of the principal axes of the reciprocal strain ellipsoid. The maximum $\left(\lambda_{1}\right)$, medium $\left(\lambda_{2}\right)$, and minimum eigenvalue $\left(\lambda_{3}\right)$ are called the maximum, medium, and minimum principal strain, respectively. The maximum principal strain relates to maximum elongation and the minimum strain relates to maximum compression

\subsection{Visualization of 3-D Strain Tensor Field}

Tensor field visualization is a challenging task due in part to the multivariate nature of individual tensor samples $[31,82,178,188]$. Ellipsoids describe tensor variables by mapping the tensor eigenvectors and eigenvalues to the orientation and shape of a geometric primitive. 
A symmetric tensor such as lagrangian strain tensor can always be diagonalized, that is, decomposed into a rotation matrix, $R$, a diagonal matrix, $\Lambda$, and the inverse rotation, $R^{-1} . R$ has its column vectors made up of the eigenvectors $\left(V_{1}, V_{2}, V_{3}\right)$ and $\Lambda$ has eigenvalues $\left(\lambda_{1}, \lambda_{2}, \lambda_{3}\right)$ as the entries on the main diagonal and has zero entries elsewhere.

$$
\boldsymbol{E}=R \Lambda R^{-1}=\left[\begin{array}{lll}
V_{1} & V_{2} & V_{3}
\end{array}\right]\left[\begin{array}{ccc}
\lambda_{1} & 0 & 0 \\
0 & \lambda_{2} & 0 \\
0 & 0 & \lambda_{3}
\end{array}\right]\left[\begin{array}{l}
V_{1} \\
V_{2} \\
V_{3}
\end{array}\right]
$$

This is the natural decomposition of a tensor quantity into orientation and shape information. As shown in Figure 5.2, the orientation of the ellipsoid axes conveys the eigenvectors, and the scaling along the axes corresponds to the eigenvalues. The anisotropy of a tensor expresses the amount of variation in the eigenvalues. If tensors do not have any anisotropy $\left(\lambda_{1}=\lambda_{2}=\lambda_{3}\right)$, then they are considered spherical in shape. Anisotropic diffusion tensors can have linear shapes $\left(\lambda_{1}>\lambda_{2}=\lambda_{3}\right)$, planar shapes $\left(\lambda_{1}=\lambda_{2}>\lambda_{3}\right)$, or some combinations [82].

\subsection{Relationship between Jacobian and Principal Strains}

Since the inverse of a rotation matrix is equals to its transpose, we have the following relations:

$$
\begin{aligned}
& \boldsymbol{E}=\frac{1}{2}\left(\boldsymbol{F}^{T} \boldsymbol{F}-\boldsymbol{I}\right)=R \Lambda R^{T} \\
& \boldsymbol{F}^{T} \boldsymbol{F}=2\left(R \Lambda R^{T}+1 / 2 R R^{T}\right) \\
& \boldsymbol{F}^{T} \boldsymbol{F}=2\left(R(\Lambda+1 / 2 \boldsymbol{I}) R^{T}\right)
\end{aligned}
$$

Since $\boldsymbol{F}$ is a $3 \times 3$ tensor, applying the determinant to both sides of the above equality yields:

$$
(\operatorname{det}(\boldsymbol{F}))^{2}=8 \operatorname{det}(\Lambda+1 / 2 \boldsymbol{I})
$$

As $J=\operatorname{det}(\boldsymbol{F})$,

$$
J=\sqrt{\left(2 \lambda_{1}+1\right)\left(2 \lambda_{2}+1\right)\left(2 \lambda_{3}+1\right)}
$$

Therefore, Jacobian of deformation can be computed from the principal strain values. 


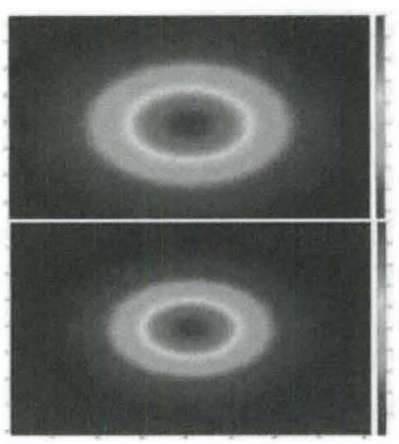

(a)

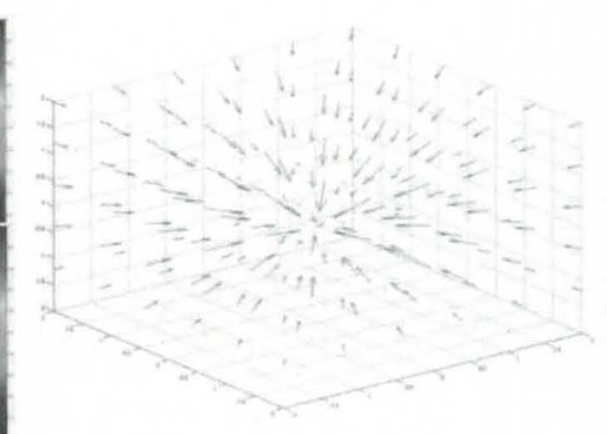

(b)

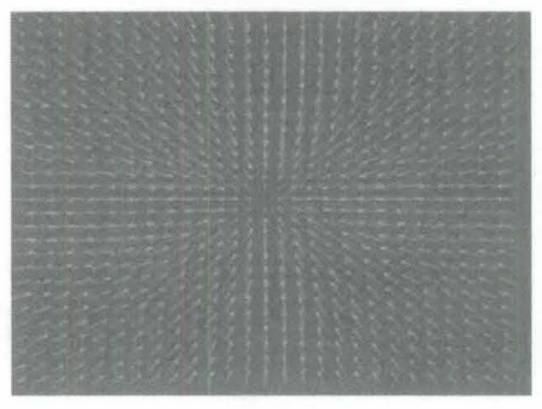

(c)

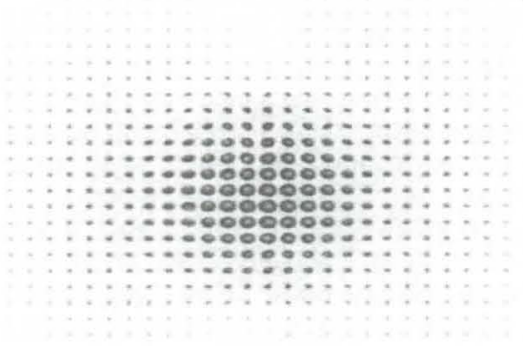

(d)

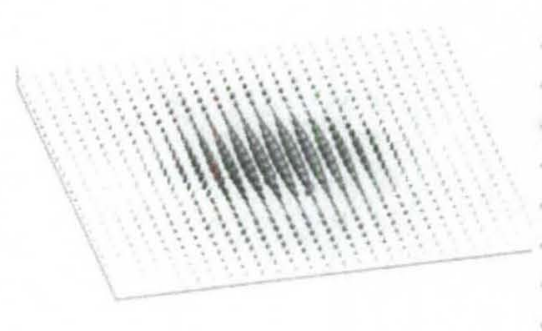

(e)

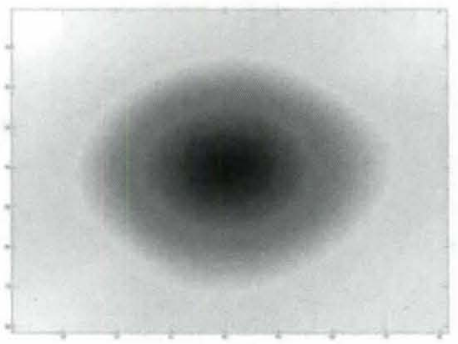

(f)

Figure 5.3: (a) Cross section of a contracting volumetric sponge $I(x, y, z, t)=\exp \left(-(x-\mu t)^{2}-\right.$ $\left.(y-v t)^{2}-(z-\omega t)^{2}\right)$ for time points $t=1$ and $t=1.1$. (b) The three dimensional calculated motion field. (c) Axial cross section of the calculated motion field in (b) corresponding to the same axial location shown in (a). (d) Cross section of the glyph ellipsoid representation of the calculated strain tensor, same cross section as in (a). (e) The same display in (d) but shown from a different view point. (f) Minimum principal component (contraction component) of the calculated strain tensor corresponding to the cross sections displayed in (a).

\subsection{Experimental Results}

Since the respiratory motion of the lung can be simplified and modeled as a time varying contraction and expansion [116], a volumetric sponge model which undergoes contraction and expansion has been used to evaluate the accuracy of the strain calculation method. Figure 5.3 displays the estimated motion and the calculated strain in glyph ellipsoid representation for a contracting sponge. 


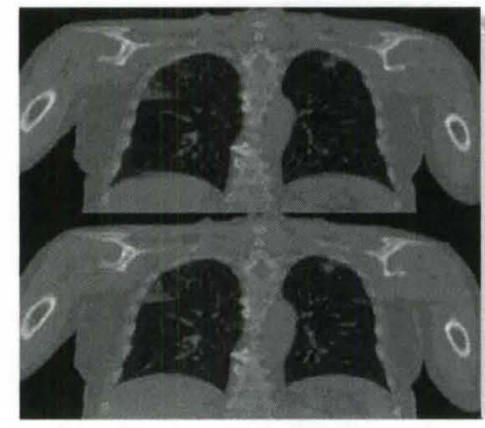

(a)

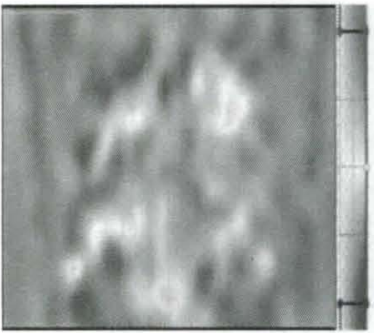

(d)

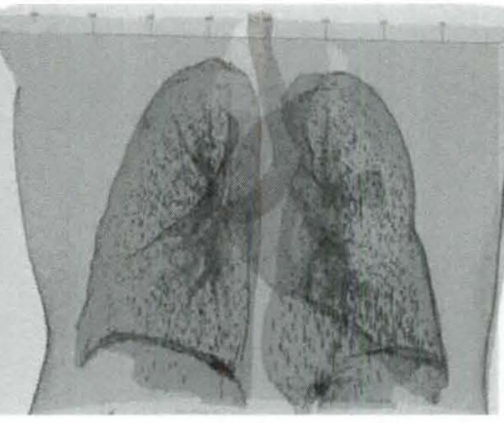

(b)

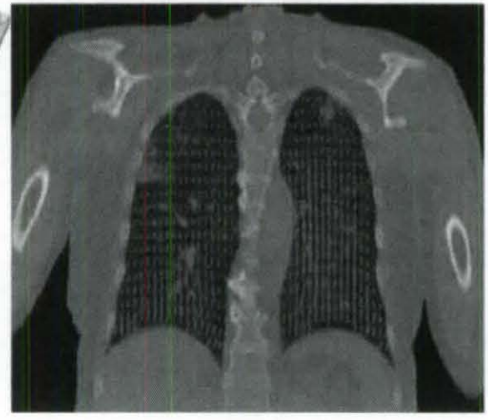

(c)

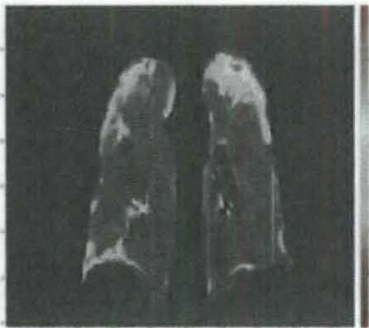

(e)

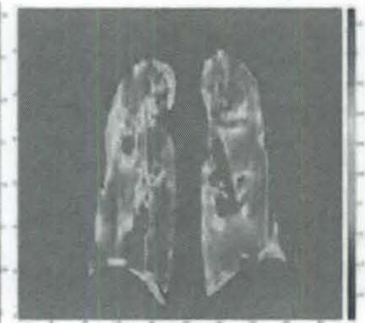

(f)

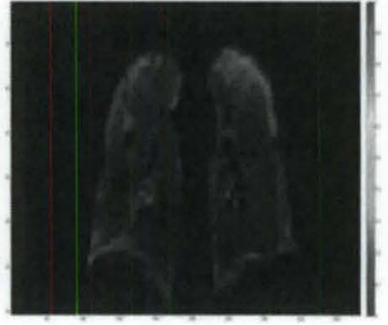

(g)

Figure 5.4: (a) Coronal plane of the POPI-model in max-inhale (top) and max-exhale (bottom) respiration phase. (b) The calculated volumetric motion field. (c) The coronal plane of the calculated motion field. (d) A color coded Jacobian image with the scale going from 0.3 (blue) to 1.7 (red). (e) The maximum principal strain component. (f) The minimum principal strain component. (g) The superimposition of both maximum (red) and minimum (blue) principal strain components.

Figure 5.4 displays results from two volumetric images between phases P30 and P40 of the POPImodel. To further evaluate correctness of the deformable registration, singularity assessment was performed in order to ensure that registrations represented meaningful and physically plausible deformations. Figure 5.4(d) shows color-coded Jacobian of the POPI-model data between P30 and P40. As expected, there is no singularity in the calculated registration. Figure 5.4(e) and 5.4(f) display the maximum and minimum 3-D principal strains illustrated for the mid-coronal slice of Figure 5.4(a). In Figure 5.4(g), the two strain images were combined using RGB channel combination with the R channel corresponding to the maximum component and B channel corresponding to minimum component. The $\mathrm{G}$ channel was set to zero.

The robustness of the proposed motion estimation and strain calculation method has been evaluated on the deforming sponge volume in the presence of Gaussian noise with different variances added to temporal 


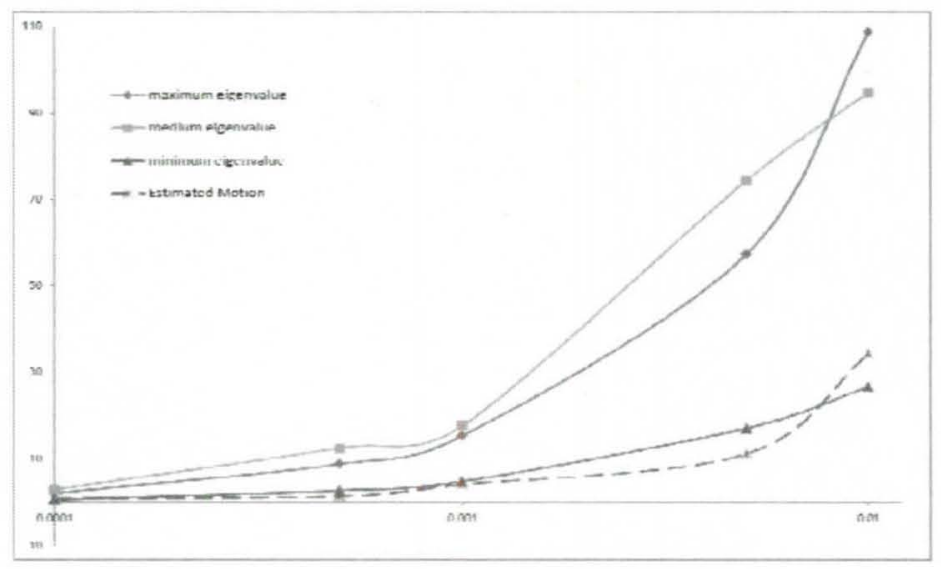

Figure 5.5: Solid lines: RRMS error between the estimated Lagrangian principal strains (maximum, medium, and minimum) and corresponding ground-truth quantities for the entire image volume. Dashed line: RRMS error between the estimated motion and the true motion. Abscissa: variance of additive Gaussian noise (Noise with a variance of 0.01 far exceeds the noise power encountered in lung CT data).

Ordinate: RRMS error in percent.

volumetric images for both times $t=1$ and $t=1.1$. Figure 5.5 shows the relative root mean squared (RRMS) error between the true and estimated motion as well as the estimated maximum, medium, and minimum principal strain and corresponding ground-truth quantities. As may be seen, the errors for the motion field are significantly smaller than those for the strain. Clearly, spatio-temporal smoothness in the optical flow energy function has alleviated the effect of noise on the estimated motion while the derivative operator in calculation of DGT has amplified the effect of noise in the calculated strain tensor. 


\section{CHAPTER 6}

\section{PHYSIOLOGIC VALIDATION OF MEASURES OF REGIONAL PULMONARY FUNCTION}

\subsection{Introduction}

Radiotherapy for thoracic malignancies can cause biomechanical (strain and elasticity) changes which alter the physiological function of the lung (uptake of $\mathrm{O} 2$ and release of $\mathrm{CO} 2$ ). The extant and severity of these alterations depend on the dose and fractionation. Hypofractionated treatment, in particular SBRT, reduces the level of normal tissue tolerance to radiation and therefore presents a higher risk to a patient care. Assessing lung function for these high risk cases with an objective method which relates biomechanical change with physiological function is essential in evaluating risk for treatment and retreatment cases. Some dose escalation studies for NSCLC found higher radiation dose associated with improved overall survival [85], while other studies demonstrated the feasibility of minimizing radiation dose to functional lung tissue with incorporation of 4-D CT derived ventilation measurements $[72,194]$.

Currently, the most prevalent approach for assessing regional lung function from 4-D CT data has been a measure of Jacobian of deformation $[20,21,35,56,137,189$, 190]. However, although the Jacobian describes regional volume changes of the lung during deformation, it lacks any consideration of directional changes of local compressions and expansions during respiration. 4-D CT measured lung strain is a quantitative method which provides regional volume changes during deformation; it provides directional changes for the local compressions and expansions during respiration.

Herein, we propose a novel approach to quantification of motion and mechanical strain in the lung from treatment planning 4-D CT images using MOFID to derive volumetric deformation maps. Principal strains of deformation computed from clinical treatment planning 4-D CT data were then correlated with both tomographic SPECT ventilation $\left(V_{S P E C T}\right)$ and tomographic SPECT perfusion $\left(Q_{S P E C T}\right)$ scans in seven lung 
cancer patients and showed quantitative results of the comparison of mechanical strain as a novel index of regional lung function with previously established Jacobian of deformation [123, 124].

\subsection{Patient Studies}

The study was approved by University of Louisville's Institutional Review Board. Seven patients with non-small cell lung cancer (NSCLC) who were scheduled to receive thoracic radiotherapy were enrolled in the study and imaging was performed prior to the initiation of treatment. 4-D CT data were collected with 3 mm slice thickness using a Philips Brilliance Big Bore CT scanner and the Varian Real-time Position Management (RPM) system (Varian Medical Systems, Palo Alto, CA) to record patient respiratory traces in the Department of Radiation Oncology at the University of Louisville. An audiovisual feedback device was utilized to ensure a reproducible and consistent respiratory cycle waveform to ensure fidelity of the 4D CT data. For each patient, 4-D CT images of the entire thorax and upper abdomen were obtained. The 4D CT image characteristics of each of the seven patients utilized in this study are given in Table 6.1.

Each patient also received tomographic (SPECT) ventilation and tomographic (SPECT) perfusion imaging on a Philips ADAC Sky-light Dual head gamma camera. For SPECT ventilation, ${ }^{99 \mathrm{~m}} \mathrm{Tc}-$ diethylenetriamine-penta-acetic acid (Tc-99m DTPA) was aerosolized and inhaled, while for SPECT

perfusion, ${ }^{99 \mathrm{~m}} \mathrm{Tc}-$ macroaggregated albumin (Tc-99m MAA) was injected intravenously. The range of inhaled Tc-99m DTPA was 21.2-25.9 mCi (mean \pm SD: $23.8 \pm 1.5 \mathrm{mCi}$ ) while the range of injected Tc99m MAA was $4.1-4.9 \mathrm{mCi}$ (mean $\pm \mathrm{SD}: 4.5 \pm 0.3 \mathrm{mCi}$ ). The SPECT scans were acquired in a $128 \times 128$ matrix with a pixel size of $4.6 \mathrm{~mm} \times 4.6 \mathrm{~mm}$. Table 6.1 shows the characteristics of patient data. Figure 6.1 shows the $V_{S P E C T}$ and $Q_{S P E C T}$ of all patients in this study at mid-coronal slice. The SPECT images have been thresholded to the 95th percentile value of intensities within the segmented lung area in order to remove the SPECT focal areas of intense tracer activities, occurring because of airway deposition of the tracer. 
Table 6.1: The characteristics of patient data included in this study

\begin{tabular}{llcccc}
\hline $\mathbf{P t}$ & Malignancy & $\begin{array}{c}\text { 4-D CT image } \\
\text { dimension }\end{array}$ & $\begin{array}{c}\text { 4-D CT voxel } \\
\text { dimension }(\mathrm{mm})\end{array}$ & $\begin{array}{c}\text { SPECT ventilation } \\
\text { image dimension }\end{array}$ & $\begin{array}{c}\text { SPECT perfusion } \\
\text { image dimension }\end{array}$ \\
\hline $\mathbf{1}$ & NSCLC & $512 \times 512 \times 104$ & $1.36 \times 1.36 \times 3$ & $128 \times 128 \times 72$ & $128 \times 128 \times 65$ \\
\hline $\mathbf{3}$ & NSCLC & $512 \times 512 \times 94$ & $1.17 \times 1.17 \times 3$ & $128 \times 128 \times 85$ & $128 \times 128 \times 84$ \\
\hline $\mathbf{4}$ & NSCLC & $512 \times 512 \times 92$ & $1.17 \times 1.17 \times 3$ & $128 \times 128 \times 79$ & $128 \times 128 \times 88$ \\
\hline $\mathbf{5}$ & NSCLC & $512 \times 512 \times 85$ & $1.17 \times 1.17 \times 3$ & $128 \times 128 \times 83$ & $128 \times 128 \times 58$ \\
\hline 7 & NSCLC & $512 \times 512 \times 71$ & $1.17 \times 1.17 \times 3$ & $128 \times 128 \times 62$ & $128 \times 128 \times 86$ \\
\hline
\end{tabular}

\subsection{Method}

\subsubsection{Deformable Image Registration}

Following 4-D CT data collection, the multi-scale optical flow deformable image registration method (MOFID), described in Chapter 3, was used to create a spatial voxel-wise deformation map that shows the motion of the lung tissue between the end of inhale and the end of exhale [119].

The volumetric images of expiratory phases are registered pairwise to calculate the deformation field between the max-inhale and max-exhale respiratory phases through composition of pairwise deformation maps. The normalized mutual information (NMI) similarity yardstick has been employed to find the optimum weighs for MOFID energy function. For all 4-D CT registrations in this study, we set the weighting constant $\beta=1, \gamma=10$, and $\alpha=110$ to weigh mass conservation constraint, gradient constancy constraint, and regularization ter. Figure 6.2 illustrates the motion field as calculated by MOFID between max-inhale and max-exhale for Patient 1 in the study. 


\subsubsection{Lung Parenchyma Segmentation}

A level-set segmentation with region competition with ITK-SNAP $[150,200]$ was applied to delineate lung voxels from the $\mathrm{CT}$ images. For all cases, the primary lung tumor volume was excluded from the lung mask. Figure 6.3 shows the volumetric segmented lung from the 4-D CT images for all patients in this study.

\subsubsection{Measures of Regional Function from Deformation Maps}

Subsequently, the 4-D CT derived ventilation images were created as follows; the Jacobian of the deformation field which represents the local tissue expansion, was calculated for each voxel in the lung volume. We will refer to Jacobian maps calculated from MOFID as $V_{J a c}$. Figure 6.4 shows the calculated Jacobian map of Patient 1 at mid-axial, mid-coronal, and mid-sagittal plane. For more intuitive realization, we define the Jacobian metric of ventilation to be:

$$
V_{j a c}^{\prime}=J-1
$$

This is defined at each inhale voxel position. In Equation (6-1), positive values indicate local expansion and negative values indicate local contraction.

The strain tensor map of the deformation field was also calculated for each voxel in the lung. Figure 6.5 shows the principal strain values for the deformation between max-inhale and max-exhale calculated from 4-D CT data of Patient 1 at mid-axial, mid-coronal, and mid-sagittal plane.

\subsubsection{Co-Registration of CT and SPECT Data}

Figure 6.6 shows the SPECT ventilation image $\left(V_{S P E C T}\right)$ and SPECT perfusion image $\left(Q_{S P E C T}\right)$ of Patient 1. As shown in Figure 6.6, $Q_{S P E C T}$ shows asymmetric and heterogeneous perfusion throughout the lung, which is essentially identical to ventilation distribution of the lung. Having calculated the 4-D CT derived images, they were separately registered with SPECT ventilation and SPECT perfusion maps with an affine transformation using elastix [84]. For the affine transformation, the proposed large displacement registration framework in Chapter 4 was adopted where mutual information has been employed as a similarity metric and an adaptive stochastic gradient descent technique has been used as the optimizer. Figure 6.7 shows the SPECT ventilation image $\left(V_{S P E C T}\right)$ for Patient 1 along with the 4-D CT derived 
measures $\left(V_{j a c}, V_{\lambda_{1}}, V_{\lambda_{2}}, V_{\lambda_{3}}\right)$ registered on SPECT ventilation space. Similarly, Figure 6.8 shows the SPECT perfusion image $\left(Q_{S P E C T}\right)$ for Patient 1 along with the 4-D CT derived measures $\left(V_{J a c}, V_{\lambda_{1}}, V_{\lambda_{2}}, V_{\lambda_{3}}\right)$ registered on SPECT perfusion space.

\subsubsection{Statistical Analysis}

Finally, the physiologic accuracy of registered 4-D CT ventilation maps (i.e., Jacobian and principal strains) was quantified by comparison with both SPECT ventilation images $\left(V_{S P E C T}\right)$ and SPECT perfusion images $\left(Q_{S P E C T}\right)$, used as ground truth for measuring the pulmonary function.

The Spearman's rank correlation coefficient $(\rho)$ was determined in order to quantify the correlation between each of the 4-D CT derived ventilation images and the SPECT ventilation/perfusion images. Spearman's rank correlation coefficient is a non-parametric measure of statistical dependence between two images. The Pearson's linear correlation coefficient $(P C C)$ was also calculated as a measure of the strength of linear dependence between each of the 4-D CT derived ventilation images and the SPECT ventilation/perfusion images. The least squares regression between each of the 4-D CT derived ventilation image values and the SPECT ventilation/perfusion values were also plotted.

\subsection{Results}

Table 6.2 shows the Spearman's correlation coefficient $(\rho)$ and Pearson's linear correlation coefficient (PCC) between each of the 4-D CT derived ventilation images and $V_{S P E C T}$ for all seven subjects in this study. Similarly, Table 6.3 shows the Spearman's correlation coefficient $(\rho)$ and Pearson's linear correlation coefficient $(P C C)$ between each of the 4-D CT derived ventilation images and $Q_{S P E C T}$ for all subjects. 
Table 6.2: The Spearman's correlation coefficients, $\rho$, and Pearson's linear correlation coefficient, PCC, between SPECT ventilation $\left(V_{S P E C T}\right)$ and 4-D CT derived ventilation images for seven subjects with nonsmall cell primary lung cancer. The strongest correlation values have been bolded in each row and the best correlation, $\rho$, has been highlighted for each patient

\begin{tabular}{|c|c|c|c|c|c|c|c|c|c|}
\hline $\mathbf{P t}$ & & $V_{\text {Jac }}$ & $\begin{array}{c}p- \\
\text { value }\end{array}$ & $V_{\lambda_{1}}$ & p-value & $V_{\lambda_{2}}$ & $\begin{array}{c}\mathrm{p} \text { - } \\
\text { value }\end{array}$ & $V_{\lambda_{3}}$ & $\mathrm{p}$-value \\
\hline \multirow{2}{*}{1} & $\rho$ & -0.1948 & 0 & -0.3186 & 0 & -0.1711 & $<0.001$ & -0.0302 & $<0.001$ \\
\hline & $P C C$ & -0.1222 & $<0.001$ & -0.2859 & 0 & -0.1311 & $<0.001$ & 0.0853 & $<0.001$ \\
\hline \multirow{2}{*}{2} & $\rho$ & 0.1224 & $<0.001$ & -0.1408 & $<0.001$ & -0.0264 & $<0.001$ & 0.0878 & 0 \\
\hline & $P C C$ & 0.1342 & $<0.001$ & -0.1333 & $<0.001$ & 0.0066 & 0.14 & 0.0027 & 0.54 \\
\hline \multirow{2}{*}{3} & $\rho$ & -0.1772 & $<0.001$ & -0.3203 & 0 & -0.1909 & $<0.001$ & -0.0287 & $<0.001$ \\
\hline & PCC & -0.0978 & $<0.001$ & -0.1919 & $<0.001$ & -0.0703 & $<0.001$ & 0.0039 & 0.44 \\
\hline \multirow{2}{*}{4} & $\rho$ & 0.022 & $<0.001$ & -0.1672 & $<0.001$ & -0.0637 & $<0.001$ & 0.1308 & $<0.001$ \\
\hline & $P C C$ & 0.0357 & $<0.001$ & -0.1373 & $<0.001$ & -0.0205 & $<0.001$ & 0.1436 & $<0.001$ \\
\hline \multirow{2}{*}{5} & $\rho$ & -0.1978 & $<0.001$ & -0.3213 & 0 & -0.1616 & $<0.001$ & 0.0426 & $<0.001$ \\
\hline & PCC & -0.1332 & $<0.001$ & -0.2519 & 0 & 0.1162 & $<0.001$ & -0.0062 & $<0.001$ \\
\hline \multirow{2}{*}{6} & $\rho$ & -0.0992 & $<0.001$ & -0.3761 & 0 & -0.0791 & $<0.001$ & -0.0007 & 0.86 \\
\hline & $P C C$ & -0.0272 & $<0.001$ & -0.3449 & 0 & 0.001 & 0.8 & 0.1424 & $<0.001$ \\
\hline \multirow{2}{*}{7} & $\rho$ & 0.0653 & $<0.001$ & -0.1643 & 0 & 0.1115 & $<0.001$ & 0.1183 & $<0.001$ \\
\hline & $P C C$ & 0.0848 & $<0.001$ & -0.1315 & $<0.001$ & 0.0632 & $<0.001$ & 0.1499 & 0 \\
\hline
\end{tabular}


Table 6.3: The Spearman's correlation coefficients, $\rho$, and Pearson's linear correlation coefficient, $P C C$, between SPECT perfusion $\left(Q_{S P E C T}\right)$ and 4-D CT derived ventilation images for seven subjects with nonsmall cell primary lung cancer. The strongest correlation values have been bolded in each row and the best correlation, $\rho$, has been highlighted for each patient

\begin{tabular}{|c|c|c|c|c|c|c|c|c|c|}
\hline $\mathrm{Pt}$ & & $V_{\text {Jac }}$ & $\begin{array}{l}\mathrm{p}^{-} \\
\text {value }\end{array}$ & $V_{\lambda_{1}}$ & $\mathrm{p}$-value & $V_{\lambda_{2}}$ & $\begin{array}{l}\mathrm{p}- \\
\text { value }\end{array}$ & $V_{\lambda_{3}}$ & p-value \\
\hline \multirow{2}{*}{1} & $\rho$ & -0.179 & 0 & -0.319 & 0 & -0.1774 & 0 & -0.0113 & 0.01 \\
\hline & $P C C$ & -0.1349 & $<0.001$ & -0.305 & 0 & -0.1516 & $<0.001$ & 0.086 & $<0.001$ \\
\hline \multirow{2}{*}{2} & $\rho$ & 0.0147 & 0.001 & -0.2303 & 0 & -0.0658 & $<0.001$ & 0.2111 & 0 \\
\hline & $P C C$ & 0.0625 & $<0.001$ & -0.1891 & 0 & -0.0257 & $<0.001$ & 0.0007 & 0.87 \\
\hline \multirow{2}{*}{3} & $\rho$ & -0.0564 & $<0.001$ & -0.174 & $<0.001$ & -0.1148 & $<0.001$ & 0.0481 & $<0.001$ \\
\hline & $P C C$ & -0.0488 & $<0.001$ & -0.1529 & $<0.001$ & -0.0678 & $<0.001$ & -0.0014 & 0.79 \\
\hline \multirow{2}{*}{4} & $\rho$ & -0.1003 & $<0.001$ & -0.3238 & 0 & -0.1826 & $<0.001$ & 0.0839 & $<0.001$ \\
\hline & $P C C$ & -0.0898 & $<0.001$ & -0.2927 & 0 & -0.132 & $<0.001$ & 0.1102 & $<0.001$ \\
\hline \multirow{2}{*}{5} & $\rho$ & -0.1513 & $<0.001$ & -0.2308 & 0 & -0.1258 & $<0.001$ & 0.0406 & $<0.001$ \\
\hline & $P C C$ & -0.0876 & $<0.001$ & -0.1772 & $<0.001$ & -0.078 & $<0.001$ & -0.0057 & 0.3 \\
\hline \multirow{2}{*}{6} & $\rho$ & -0.2638 & 0 & -0.3344 & 0 & -0.1838 & 0 & -0.1868 & 0 \\
\hline & $P C C$ & -0.2076 & 0 & -0.3275 & 0 & -0.1179 & $<0.001$ & -0.0418 & $<0.001$ \\
\hline \multirow{2}{*}{7} & $\rho$ & -0.0739 & $<0.001$ & -0.1736 & 0 & 0.0214 & $<0.001$ & -0.0343 & $<0.001$ \\
\hline & $P C C$ & -0.0491 & $<0.001$ & -0.1143 & $<0.001$ & -0.0116 & 0.002 & 0.0147 & $<0.001$ \\
\hline
\end{tabular}




\subsection{Discussion}

Regional heterogeneity is apparent for 4-D CT derived images $\left(V_{J a c}, V_{\lambda_{1}}, V_{\lambda_{2}}, V_{\lambda_{3}}\right)$; however, the spatial distribution is different between the Jacobian and each of the principal strain values. By visual inspection of Figure 6.7 and Figure 6.8, agreements in some regions can be observed; however, many disagreements throughout the lung are also observed. For instance, for mid-coronal $Q_{S P E C T}$ and in the lower right region of lung, a reasonable agreement between $V_{J a c}$ and $V_{\lambda_{1}}$ and perfusion image can be seen. Similarly, $V_{S P E C T}$ and $Q_{S P E C T}$ demonstrate more activity in the right apical upper lobe, in agreement with pulmonary function maps $V_{J a c}, V_{\lambda_{1}}, V_{\lambda_{2}}$, and $V_{\lambda_{3}}$. Overall, where there is more contraction in $V_{J a c}$ and $V_{\lambda_{1}}$ there is more agreement with both the perfusion and ventilation images.

The 4-D CT derived ventilation images versus $V_{S P E C T}$ and $Q_{S P E C T}$ together with the Spearman's voxelbased correlation coefficient $(\rho)$ for all subjects are reported in Figure 6.9 - Figure 6.15. From visual inspection of the spatial distribution of 4-D CT derived images as well as from the computed Spearman's correlation coefficients and Pearson's correlation coefficient, $V_{\lambda_{1}}$ clearly and consistently shows better agreement and stronger correlation with both $V_{S P E C T}$ and $Q_{S P E C T}$. On the other hand, $V_{\lambda_{2}}$ and $V_{\lambda_{3}}$ yield smaller Spearman's correlation coefficients which imply less agreement with both $V_{S P E C T}$ and $Q_{S P E C T}$.

In comparison to previously reported studies $[20,190]$, our measure of Jacobian $\left(V_{J a c}\right)$ resulted in a better correlation with $V_{S P E C T}$ and $Q_{S P E C T}$. We attribute this to MOFID's more accurate estimation of the deformation field between respiratory phases of 4-D CT images.

In comparison to $V_{J a c}$, the proposed $V_{\lambda_{1}}$ shows stronger correlation with both $V_{S P E C T}$ and $Q_{S P E C T}$. Figure 6.16 shows the correlation value of the maximum principal strain $\left(V_{\lambda_{1}}\right)$ and Jacobian $\left(V_{J a c}\right)$ with both SPECT ventilation $\left(V_{S P E C T}\right)$ and SPECT perfusion $\left(Q_{S P E C T}\right)$ where $V_{\lambda_{1}}$ consistently had a more significant correlation with $V_{S P E C T}$ and $Q_{S P E C T}$ when compared to $V_{J a C}\left(\mathrm{p}<10^{-3}\right)$. We hypothesize that this may be due to the relation between Jacobian and principal strain values (please see the derived relation in Section 5.4). Based on Equation (5-23), all three principal strain values, $\left(\lambda_{1}, \lambda_{2}, \lambda_{3}\right)$, affect the value of the Jacobian in a non-linear fashion. Since $\lambda_{1}$ is the maximum principal strain value, it has a more significant impact on the statistical correlation of the Jacobian with both $V_{S P E C T}$ and $Q_{S P E C T}$; however, $\lambda_{2}$ and $\lambda_{3}$ being less correlated to $V_{S P E C T}$ and $Q_{S P E C T}$ weaken the correlation of Jacobian with both $V_{S P E C T}$ and $Q_{S P E C T}$. 
Relative to the rest of the patients in the study, for the patients with a better match of spatial distribution of ventilation and perfusion pattern as evident in the nuclear medicine physician's report, a stronger correlation of the 4-D CT derived images with both $V_{S P E C T}$ and $Q_{S P E C T}$ was found (see for example, correlation values in Tables 6.2 and 6.3 for the first and sixth patients). It should be noted that unlike the case of patients with diseases such as pulmonary embolism and hypopalsia/aplasia of pulmonary artery where there is a clear mismatch between $V_{S P E C T}$ and $Q_{S P E C T}$, there is no such mismatch evident in $80 \%$ of lung cancer patients $[49,111,199]$.

Overall, the 4-D CT derived ventilation images show a relatively weak correlation with both SPECT ventilation $\left(V_{S P E C T}\right)$ and SPECT perfusion $\left(Q_{S P E C T}\right)$, which conform to previous studies $[20,41,190]$. This could be due to issues present in both 4-D CT and SPECT imaging. Lung cancer patients can have irregular breathing; this leads to artifacts and degradation in the acquired 4-D CT images. Artifacts in treatment planning 4-D CT images cause inaccuracies in calculated CT ventilation images. Likewise, Tc-99m DTPA aerosols cause artifacts in SPECT images due to the central airway deposition rendering SPECT scans prone to inaccuracies. Although the correlation values between 4-D CT derived pulmonary function maps and SPECT images have been moderate, we should be cognizant of the fact that Tc-99m DTPA aerosol ventilation SPECT images are not strictly quantitative. The main area of strength for SPECT ventilation has been its ability to localize regional ventilation deficits-such as those arising in airway obstruction or chronic obstructive pulmonary disease (COPD)-potentially of benefit in image-guided radiation therapy treatment planning.

Moreover, the different spatial and perhaps temporal resolutions between 4-D CT images and SPECT scans can exacerbate this issue. All 4-D CT and SPECT acquisitions were performed separately and on different days resulting in significant differences in both location and stretching of the lung and diaphragm relative to the rib cage as well as more generally changes in lung function, although less likely.

The difference of imaged lung volumes between 4-D CT and SPECT should also be highlighted. SPECT ventilation and perfusion scans are free breathing scans, thus they are blurred average images of the ventilation and perfusion volumes of the patient that is studied. However, the 4-D CT scan is gated to RPM, and patients are coached via an audiovisual feedback to time their breathing so that their lung volume goes from the max-inhale phase to the max-exhale phase. It is therefore expected that the ventilation metrics 
between the max-inhale and max-exhale phases do not entirely match the ventilation and/or perfusion metrics as derived from SPECT images. Therefore, the apparent mismatch between 4-D CT derived ventilation maps and SPECT does not refute the hypothesis that quantitative images depicting regional ventilation from 4-D CT can indeed provide an accurate appraisal of pulmonary function for use in imageguided radiation therapy of the lung.

One of the limitations of this study is that due to lack of availability, the SPECT studies were not performed on a hybrid SPECT/CT system. Availability of such systems would surely improve the results since in that case 4-D CT studies could be registered to a breath-hold CT study from the hybrid system already registered to SPECT. Notwithstanding this, we have been able to obtain reasonable registrations between SPECT and 4-D CT studies and have demonstrated that measures of mechanical strain are indicative of pulmonary function from 4-D CT studies. 
( $\left.\boldsymbol{V}_{\text {SPECT }}\right)$

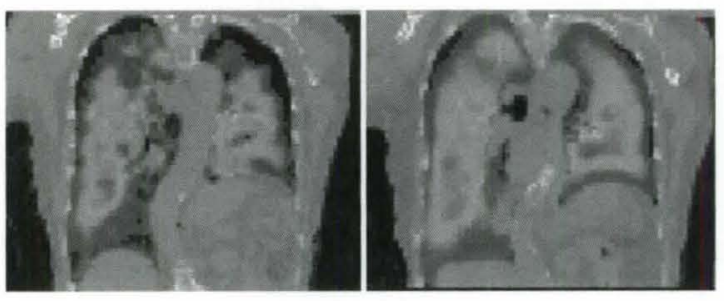

Case 1

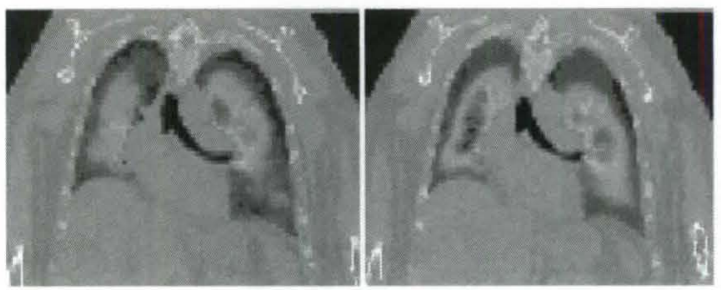

Case 3

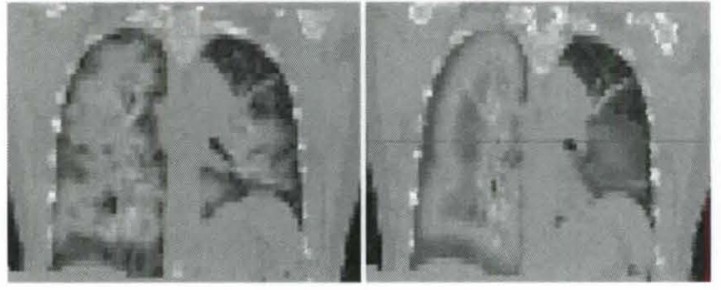

Case 5

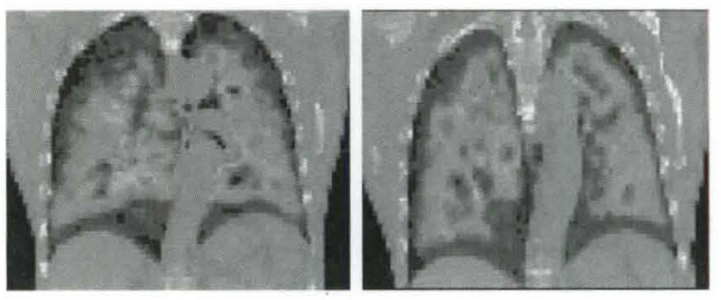

Case 7
$\left(V_{\text {SPECT }}\right)$

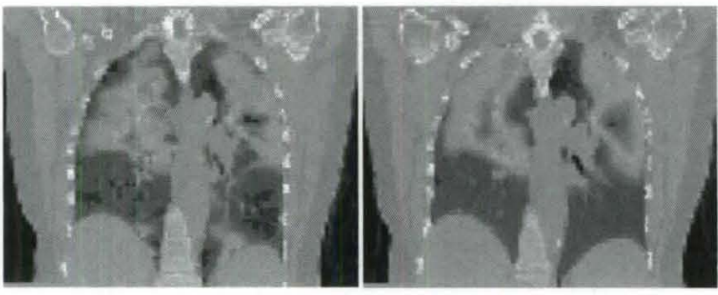

Case 2

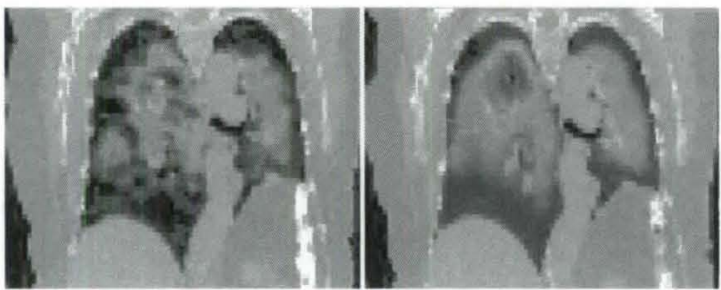

Case 4

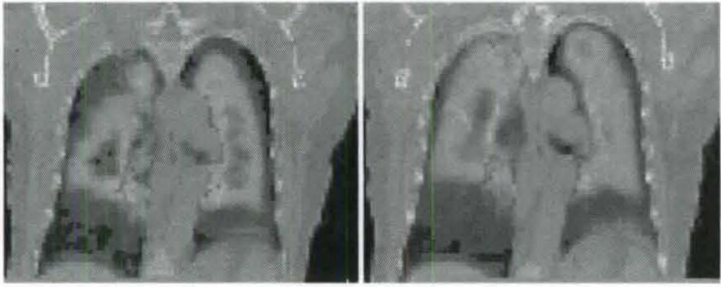

Case 6

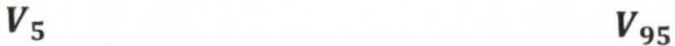

$Q_{5}$

$Q_{95}$

Figure 6.1: The SPECT ventilation image $\left(V_{S P E C T}\right)$ and SPECT perfusion image $\left(Q_{\text {SPECT }}\right)$ of all 7 patients in the study in the mid-coronal slice, shown with a scale from 5th percentile value to the 95 th percentile value. At $x$ th percentile value, $x \%$ of the total lung volume has lower value. 


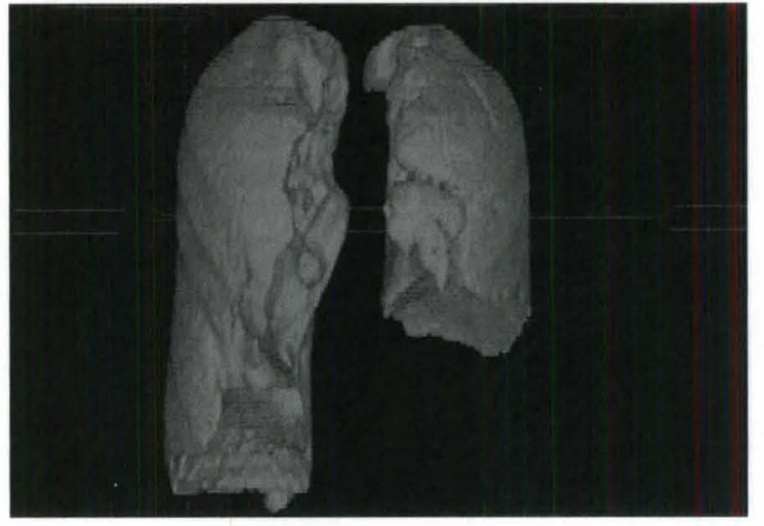

(a)

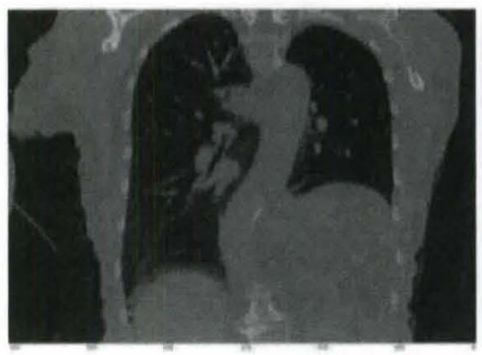

(c)

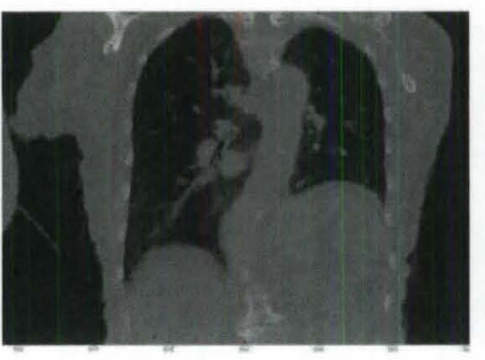

(d)

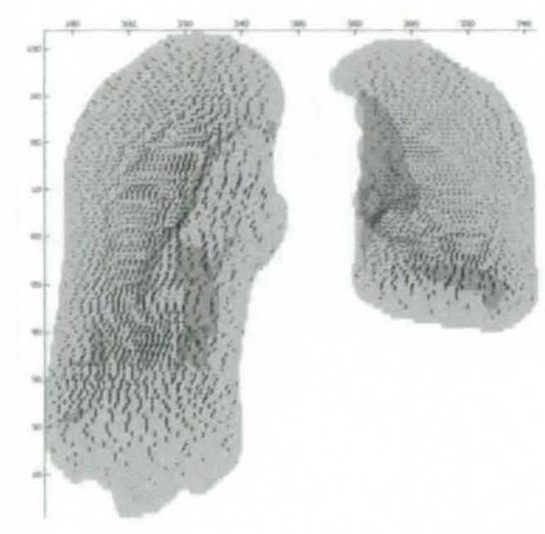

(b)

Figure 6.2: (a) Segmented lung for Patient 1. (b) The calculated volumetric motion field between endinspiration and end-expiration phases. The mid-coronal plane of the volumetric lung at end-inhale (c) and end-exhale (d). (e) The projection of the calculated motion field superimposed on max-inhale respiratory phase. 


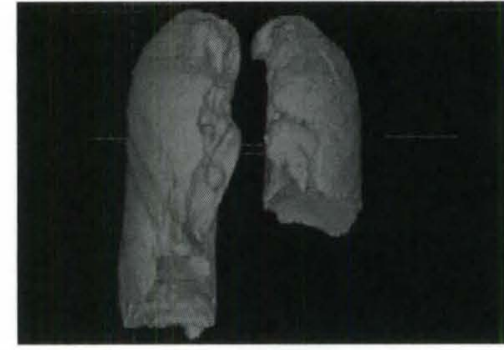

(a)

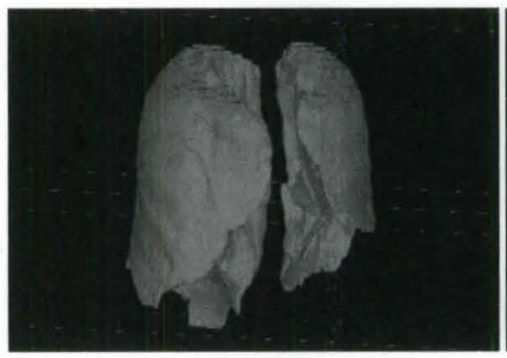

(d)

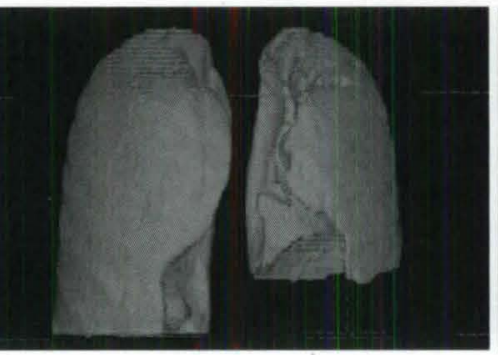

(e)

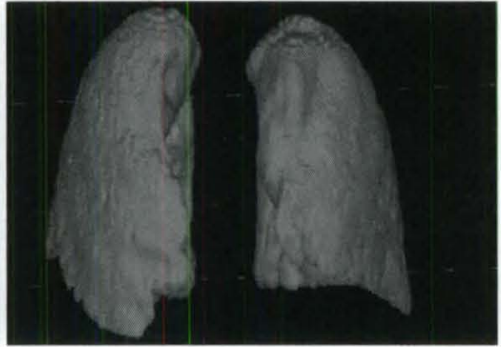

(c)

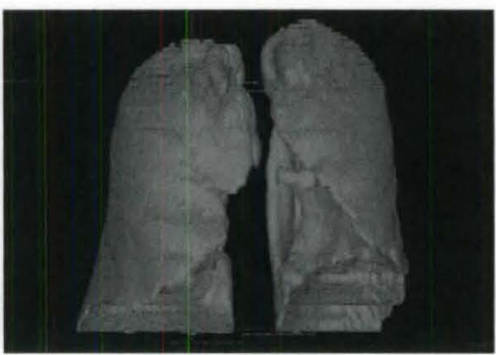

(f)

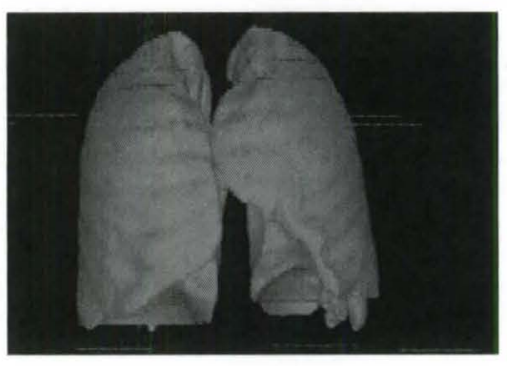

(g)

Figure 6.3: (a-g) The volumetric segmented lung from the 4-D CT images of each patient at endinspiration. 

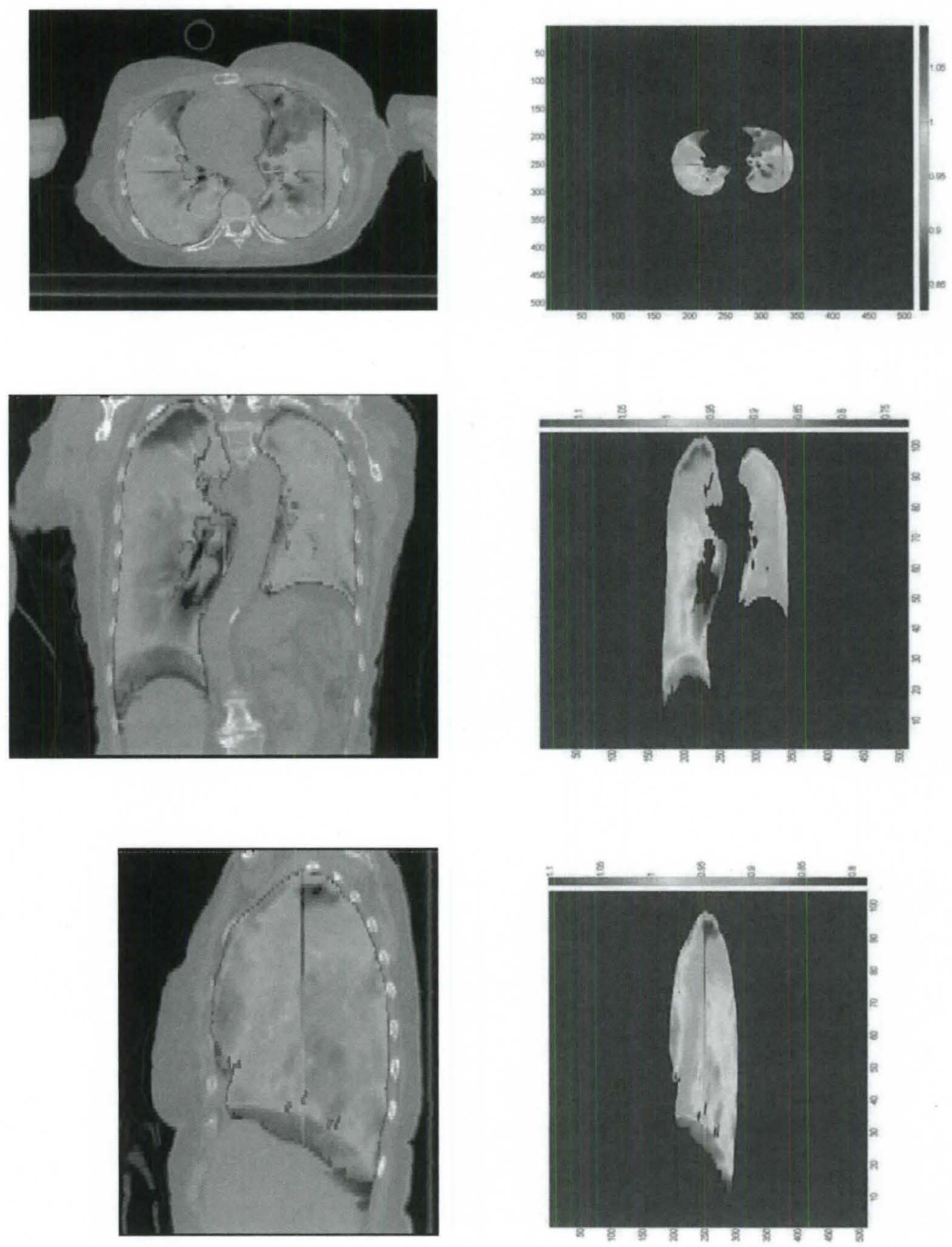

Figure 6.4: Calculated Jacobian $\left(V_{J a c}\right)$ at mid-axial, mid-coronal, and mid-sagittal slice, superimposed on end-inhale images (left column) and the quantitative images (right column). 

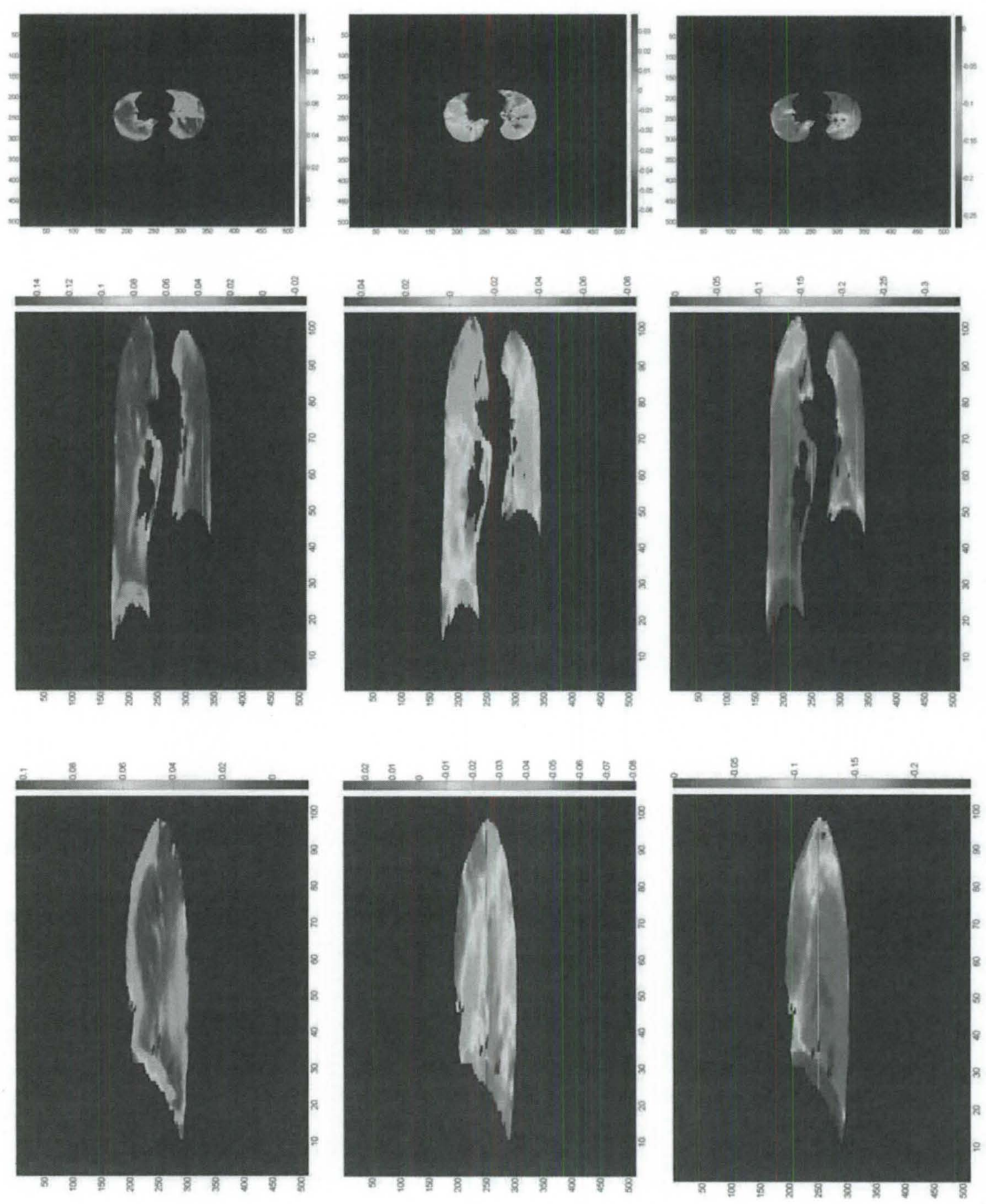

Figure 6.5: The calculated principal strain values at mid-axial (first row), mid-coronal (second row), and mid-sagittal slices (third row). The maximum principal strain image (left column), $V_{\lambda_{1}}$, the medium principal strain image (middle column), $V_{\lambda_{2}}$, and the minimum principal strain image (right column), $V_{\lambda_{3}}$. 

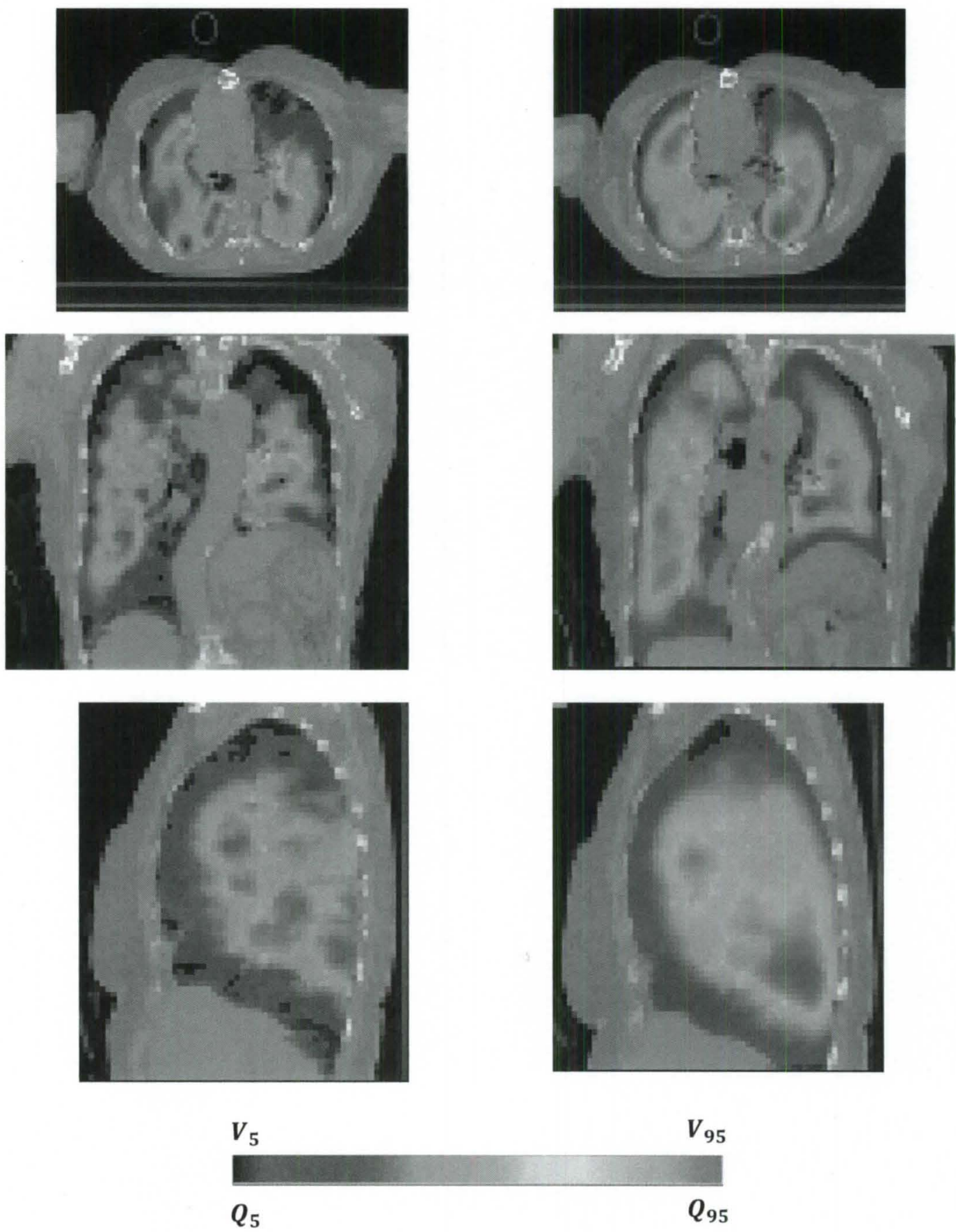

Figure 6.6: The SPECT ventilation image $\left(V_{S P E C T}\right)$ (left column) and SPECT perfusion image $\left(Q_{S P E C T}\right)$ (right column) of the first patient at mid-axial (first row), mid-coronal (second row), and mid-sagittal slice (third row). 

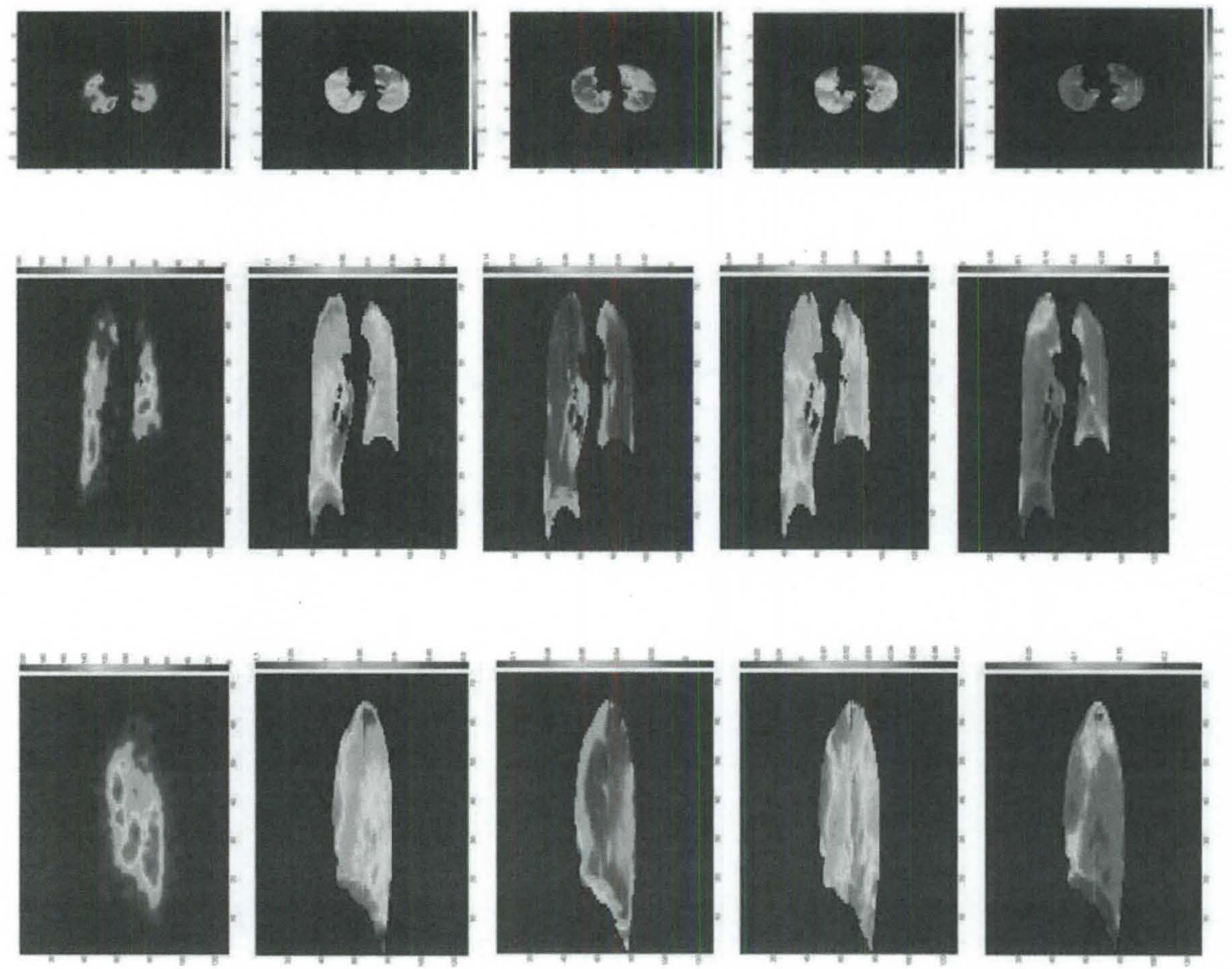

Figure 6.7: 4-D CT derived calculated images at mid-axial (first row), mid-coronal (second row), and midsagittal slice (third row) at ventilation image resolution. The SPECT ventilation image, $V_{S P E C T}$, (first column). Jacobian ventilation image, $V_{J a c}$, (second column). The maximum principal strain image, $V_{\lambda_{1}}$, (third column). The medium principal strain images, $V_{\lambda_{2}}$, (fourth column). The minimum principal strain image, $V_{\lambda_{3}}$, (fifth column). 

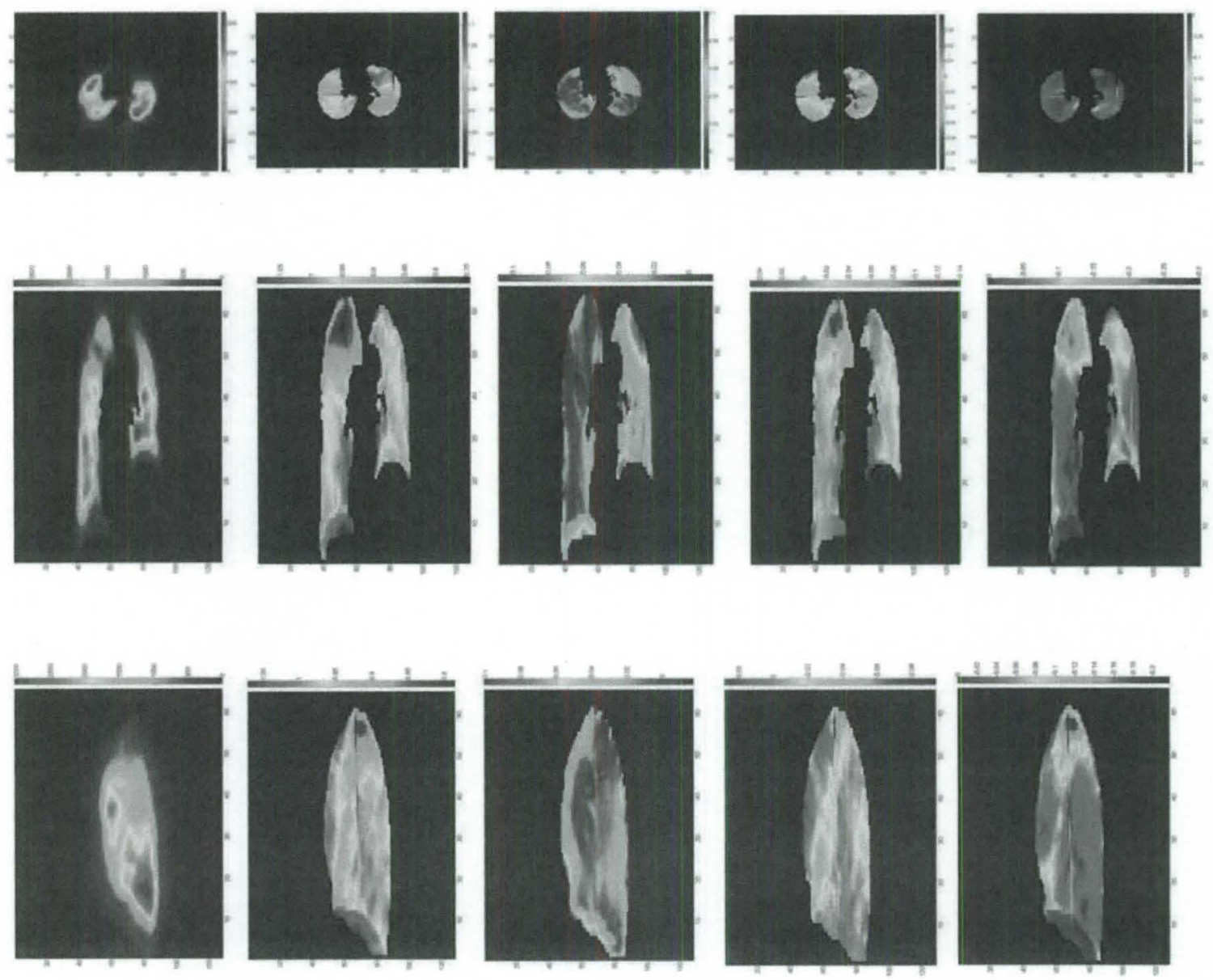

Figure 6.8: 4-D CT derived calculated images at mid-axial (first row), mid-coronal (second row), and midsagittal slice (third row) at perfusion image resolution. The SPECT perfusion image, $Q_{\text {SPECT }}$, (first column). Jacobian ventilation image, $V_{J a c}$, (second column). The maximum principal strain image, $V_{\lambda_{1}}$, (third column). The medium principal strain images, $V_{\lambda_{2}}$, (fourth column). The minimum principal strain image, $V_{\lambda_{3}}$, (fifth column). 

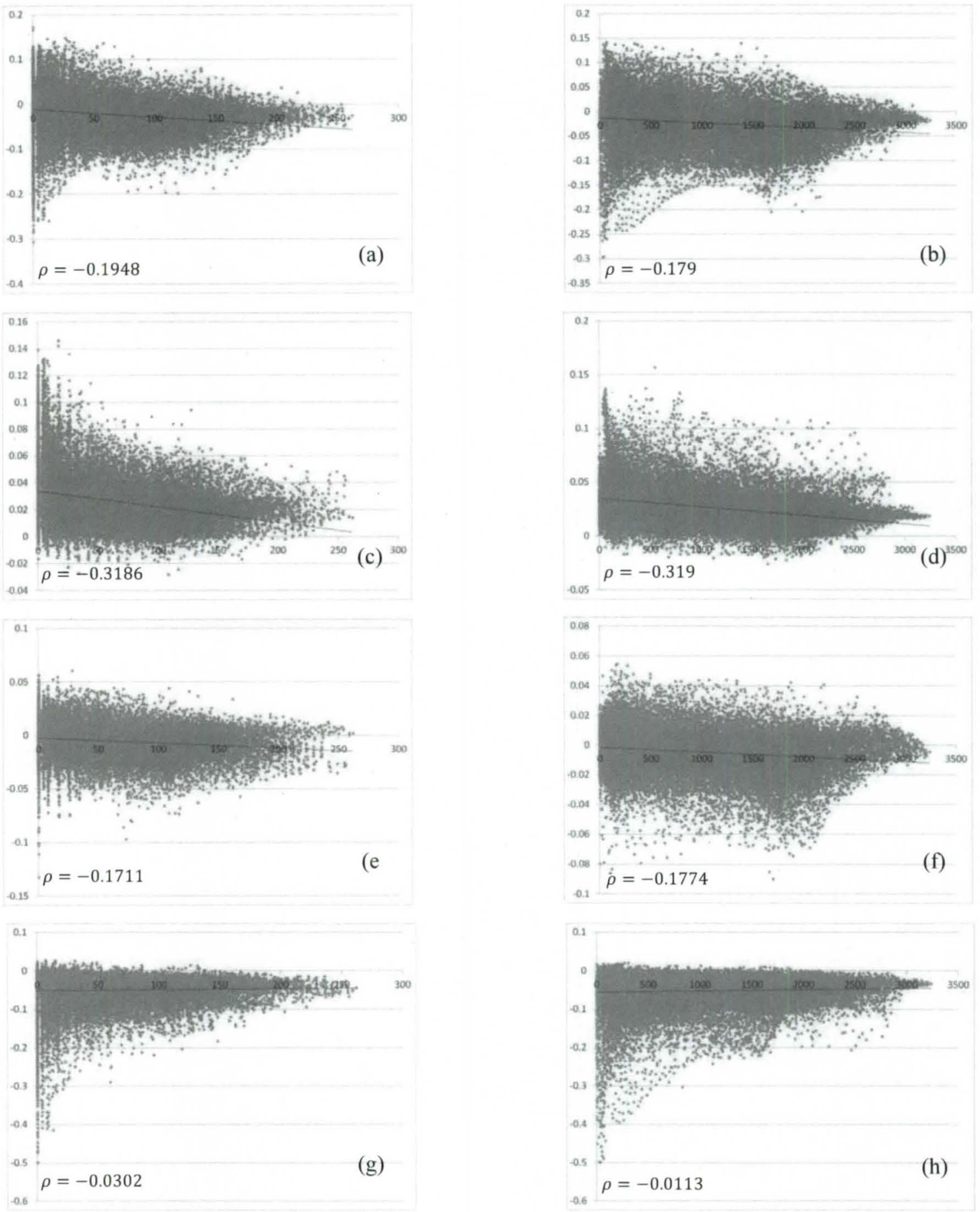

Figure 6.9: Comparison of $V_{\text {Jac }}^{\prime}$ (first row), $V_{\lambda_{1}}$ (second row), $V_{\lambda_{2}}$, (third row), and $V_{\lambda_{3}}$ (fourth row) versus SPECT ventilation, $V_{S P E C T}$, (left column) and SPECT perfusion, $Q_{S P E C T}$, (right column) in subject one. The least square regression lines and Spearman correlation coefficients, $\rho$, are also shown in each case. 

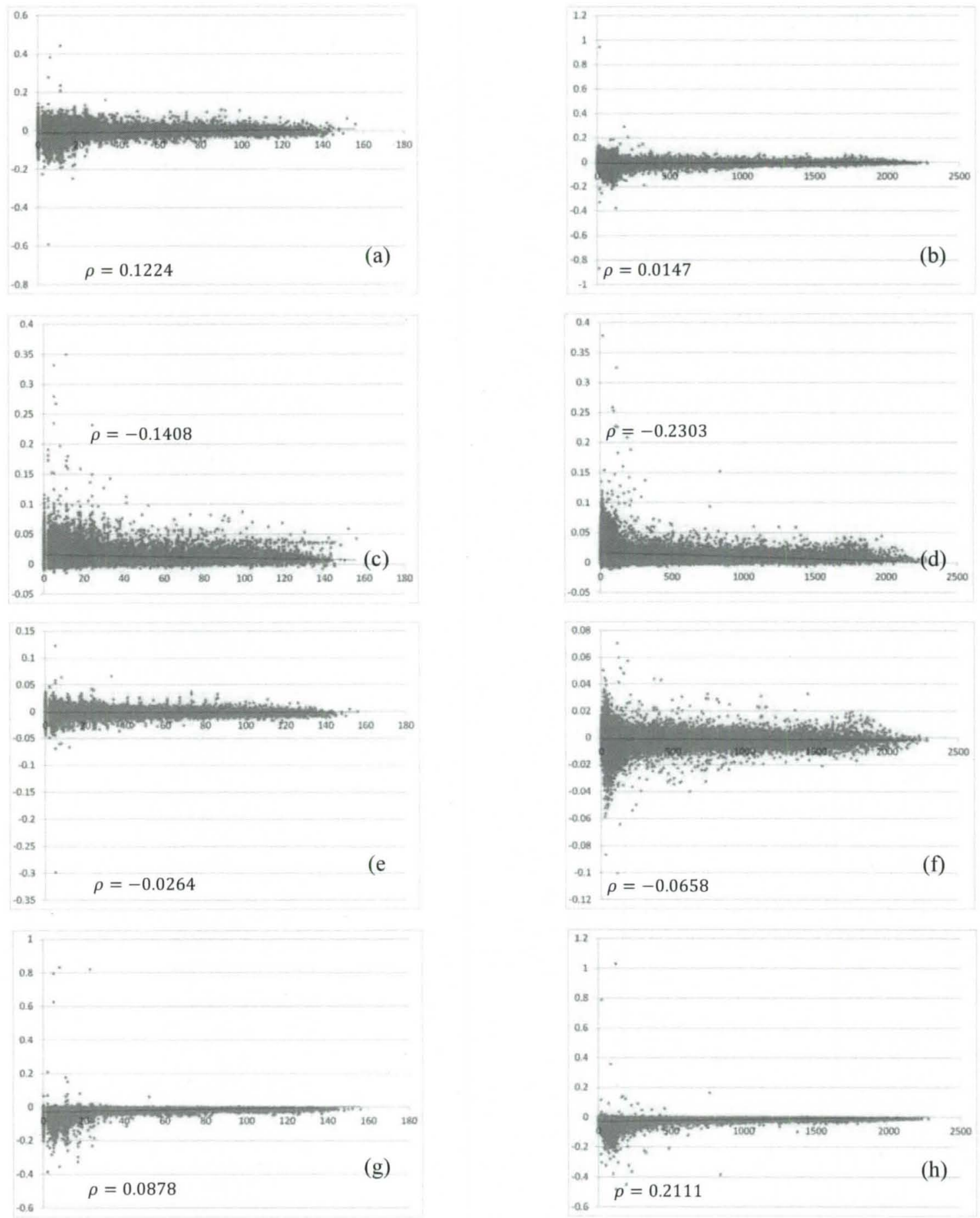

Figure 6.10: Comparison of $V^{\prime}{ }_{j a c}$ (first row), $V_{\lambda_{1}}$ (second row), $V_{\lambda_{2}}$, (third row), and $V_{\lambda_{3}}$ (fourth row) versus SPECT ventilation, $V_{S P E C T}$, (left column) and SPECT perfusion, $Q_{S P E C T}$, (right column) in subject two. The least square regression lines and Spearman correlation coefficients, $\rho$, are also shown in each case. 

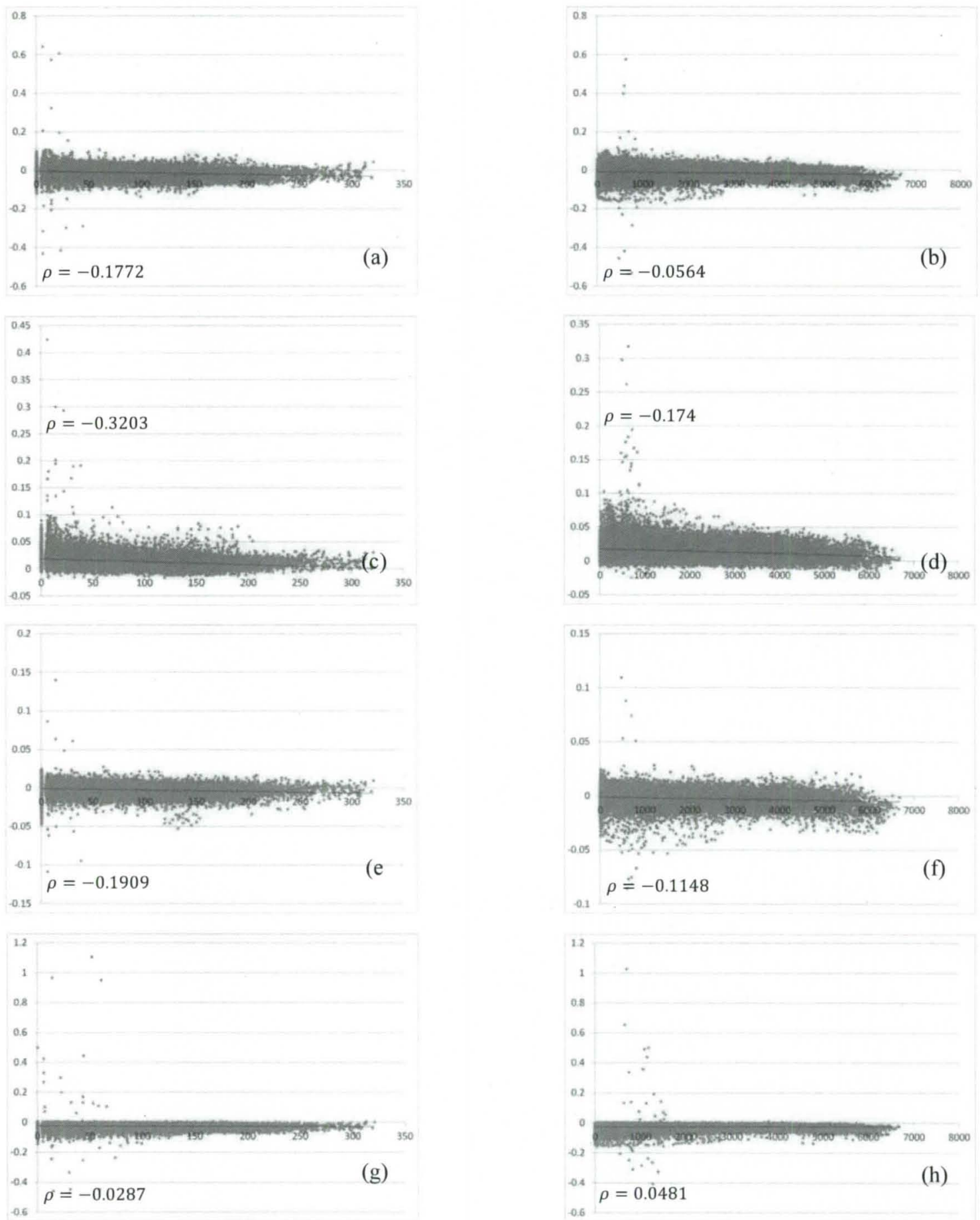

Figure 6.11: Comparison of $V_{j a c}^{\prime}$ (first row), $V_{\lambda_{1}}$ (second row), $V_{\lambda_{2}}$, (third row), and $V_{\lambda_{3}}$ (fourth row) versus SPECT ventilation, $V_{S P E C T}$, (left column) and SPECT perfusion, $Q_{S P E C T}$, (right column) in subject three. The least square regression lines and Spearman correlation coefficients, $\rho$, are also shown in each case. 

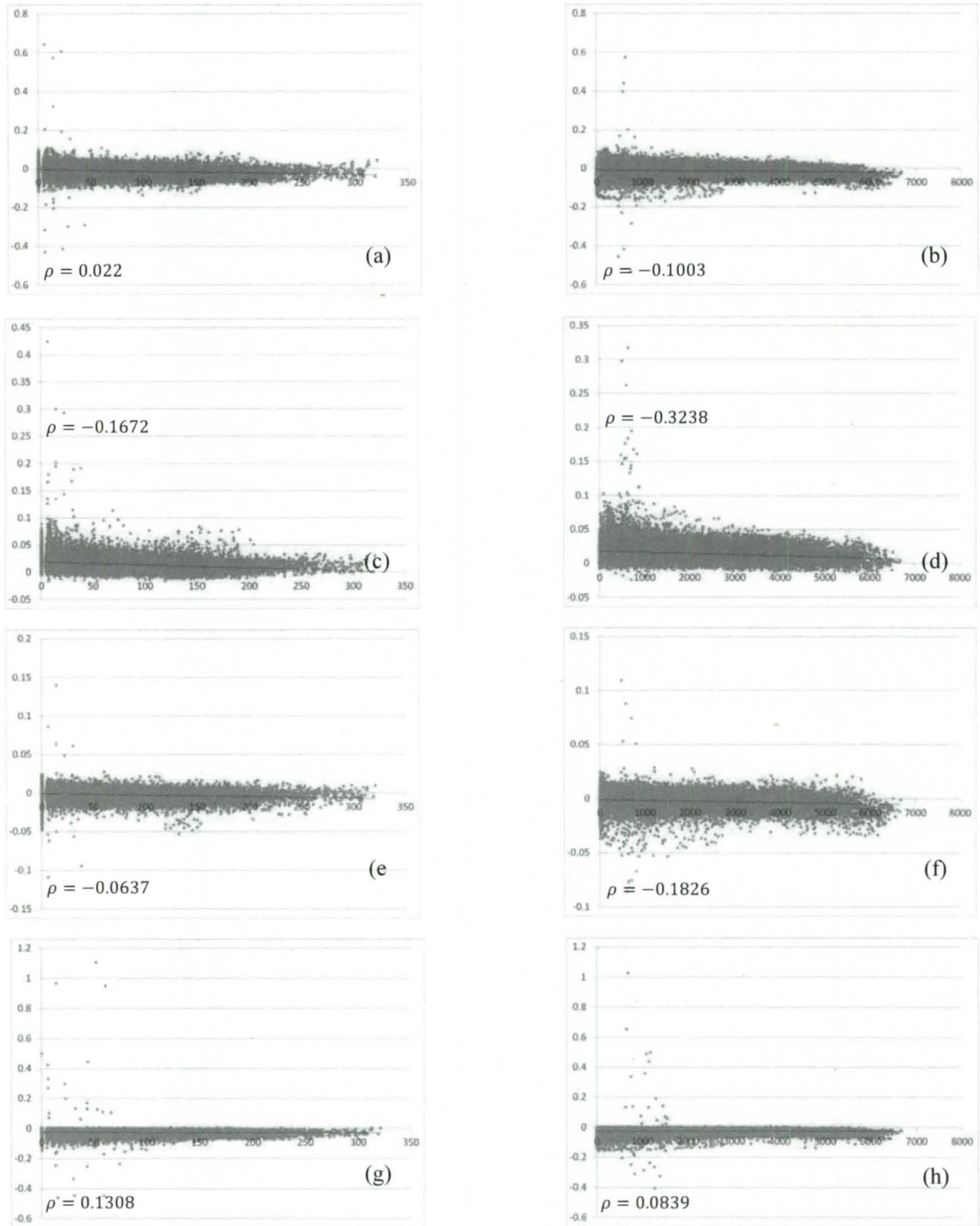

Figure 6.12: Comparison of $V_{J a c}^{\prime}$ (first row), $V_{\lambda_{1}}$ (second row), $V_{\lambda_{2}}$, (third row), and $V_{\lambda_{3}}$ (fourth row) versus SPECT ventilation, $V_{S P E C T}$, (left column) and SPECT perfusion, $Q_{S P E C T}$, (right column) in subject four. The least square regression lines and Spearman correlation coefficients, $\rho$, are also shown in each case. 

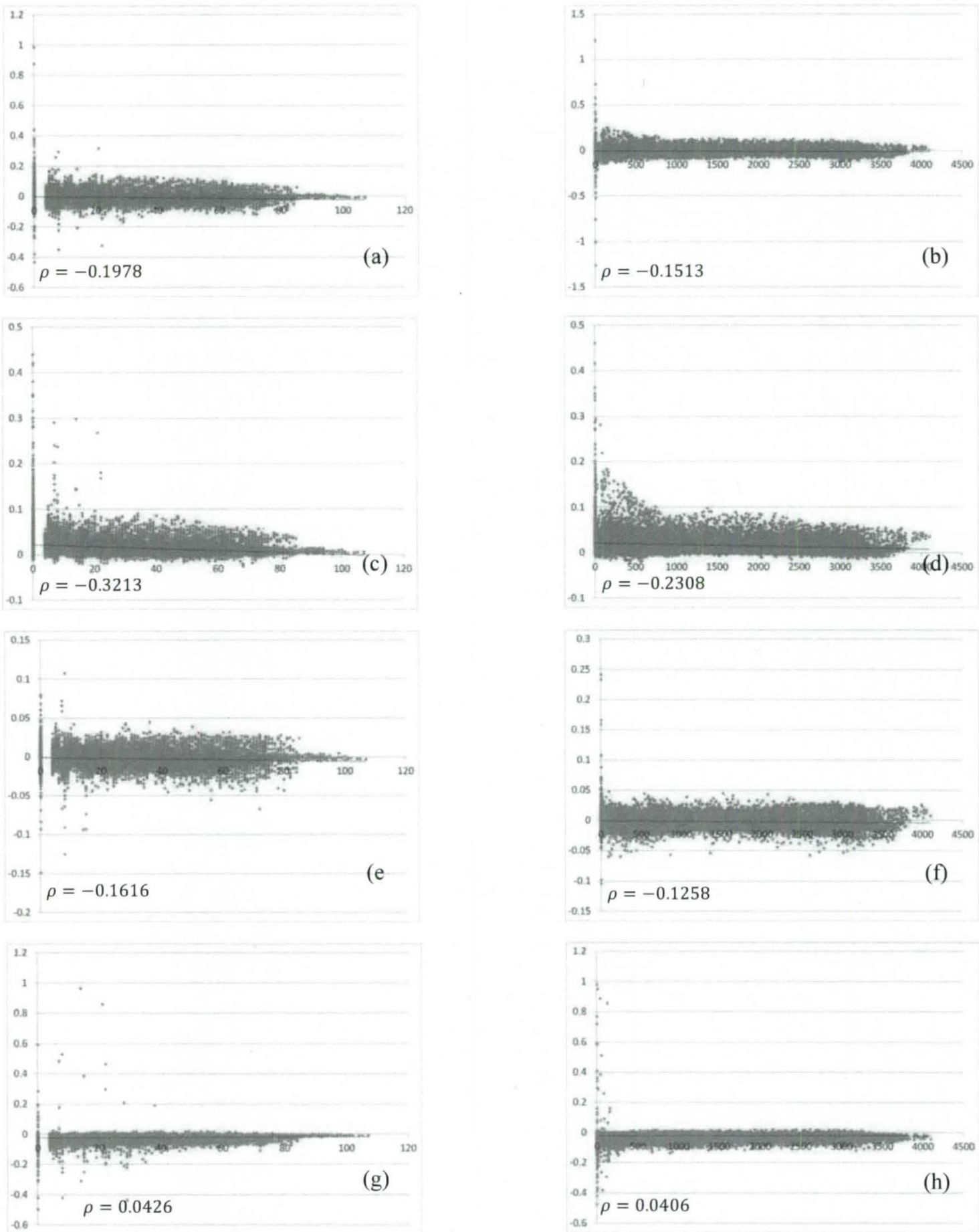

Figure 6.13: Comparison of $V_{J a c}^{\prime}$ (first row), $V_{\lambda_{1}}$ (second row), $V_{\lambda_{2}}$, (third row), and $V_{\lambda_{3}}$ (fourth row) versus SPECT ventilation, $V_{S P E C T}$, (left column) and SPECT perfusion, $Q_{S P E C T}$, (right column) in subject five. The least square regression lines and Spearman correlation coefficients, $\rho$, are also shown in each case. 

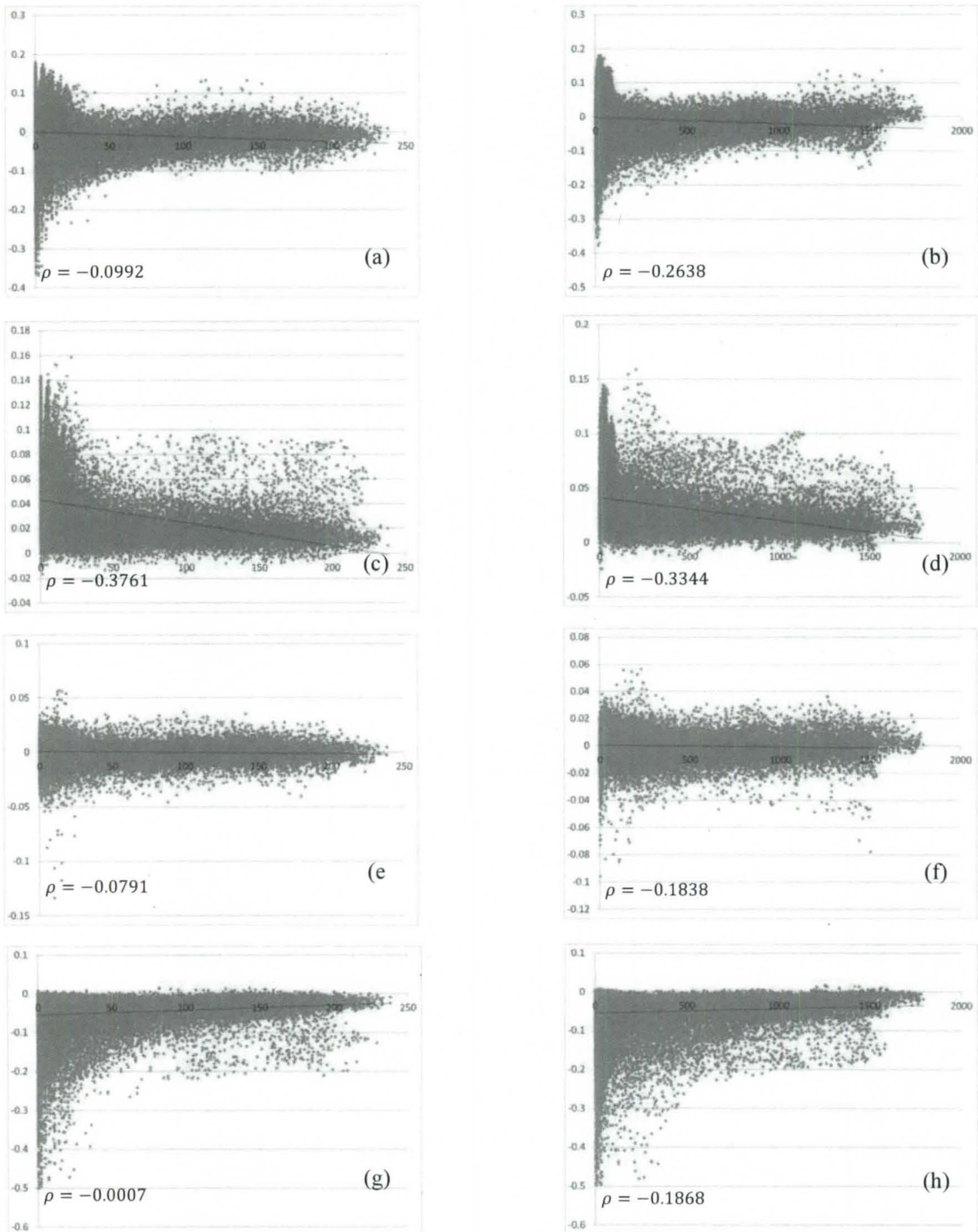

Figure 6.14: Comparison of $V^{\prime}{ }_{j a c}$ (first row), $V_{\lambda_{1}}$ (second row), $V_{\lambda_{2}}$, (third row), and $V_{\lambda_{3}}$ (fourth row) versus SPECT ventilation, $V_{S P E C T}$, (left column) and SPECT perfusion, $Q_{S P E C T}$, (right column) in subject six. The least square regression lines and Spearman correlation coefficients, $\rho$, are also shown in each case. 

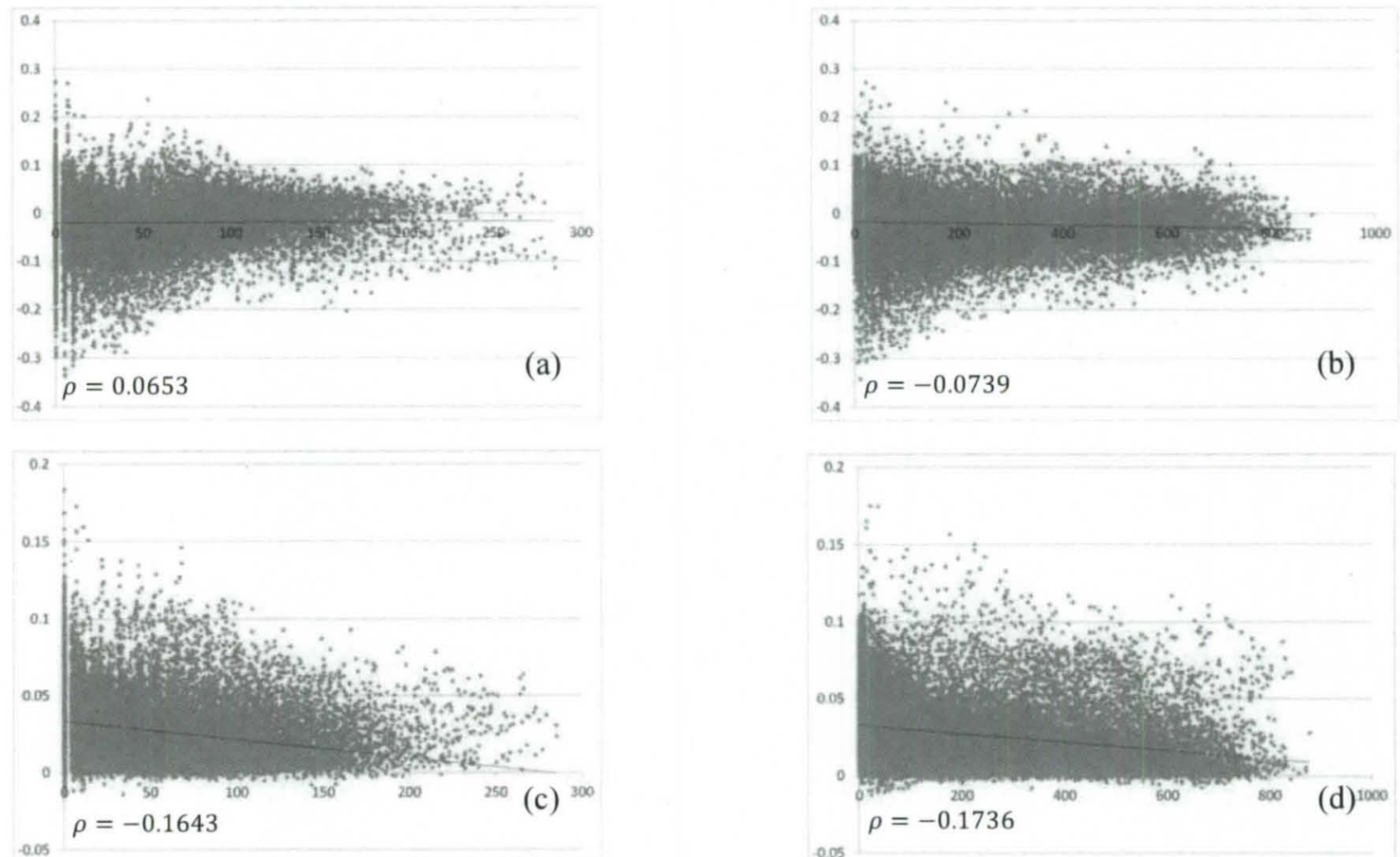

$\cos$
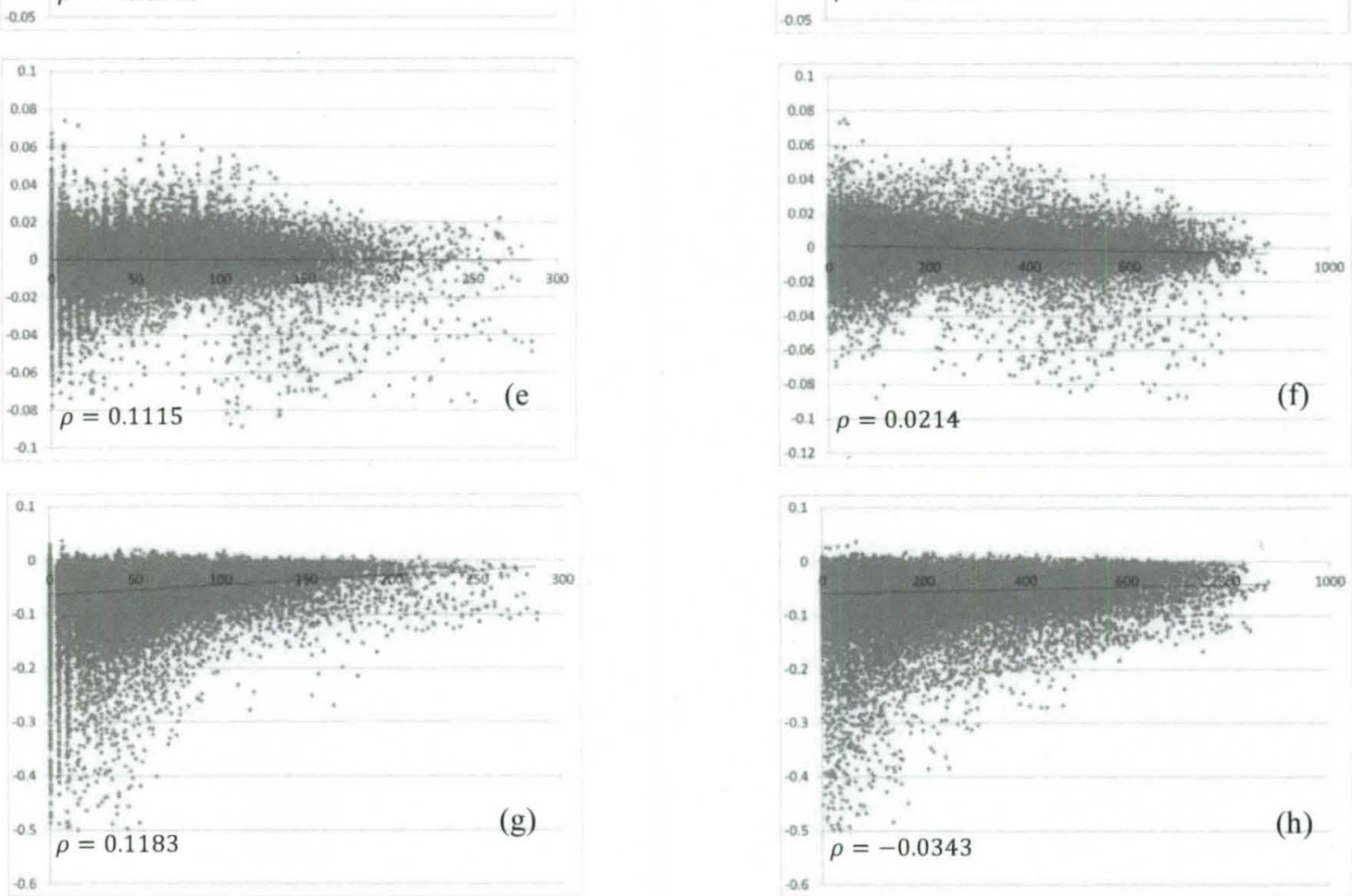

Figure 6.15: Comparison of $V_{j a c}^{\prime}$ (first row), $V_{\lambda_{1}}$ (second row), $V_{\lambda_{2}}$, (third row), and $V_{\lambda_{3}}$ (fourth row) versus SPECT ventilation, $V_{S P E C T}$, (left column) and SPECT perfusion, $Q_{S P E C T}$, (right column) in subject seven. The least square regression lines and Spearman correlation coefficients, $\rho$, are also shown in each case. 

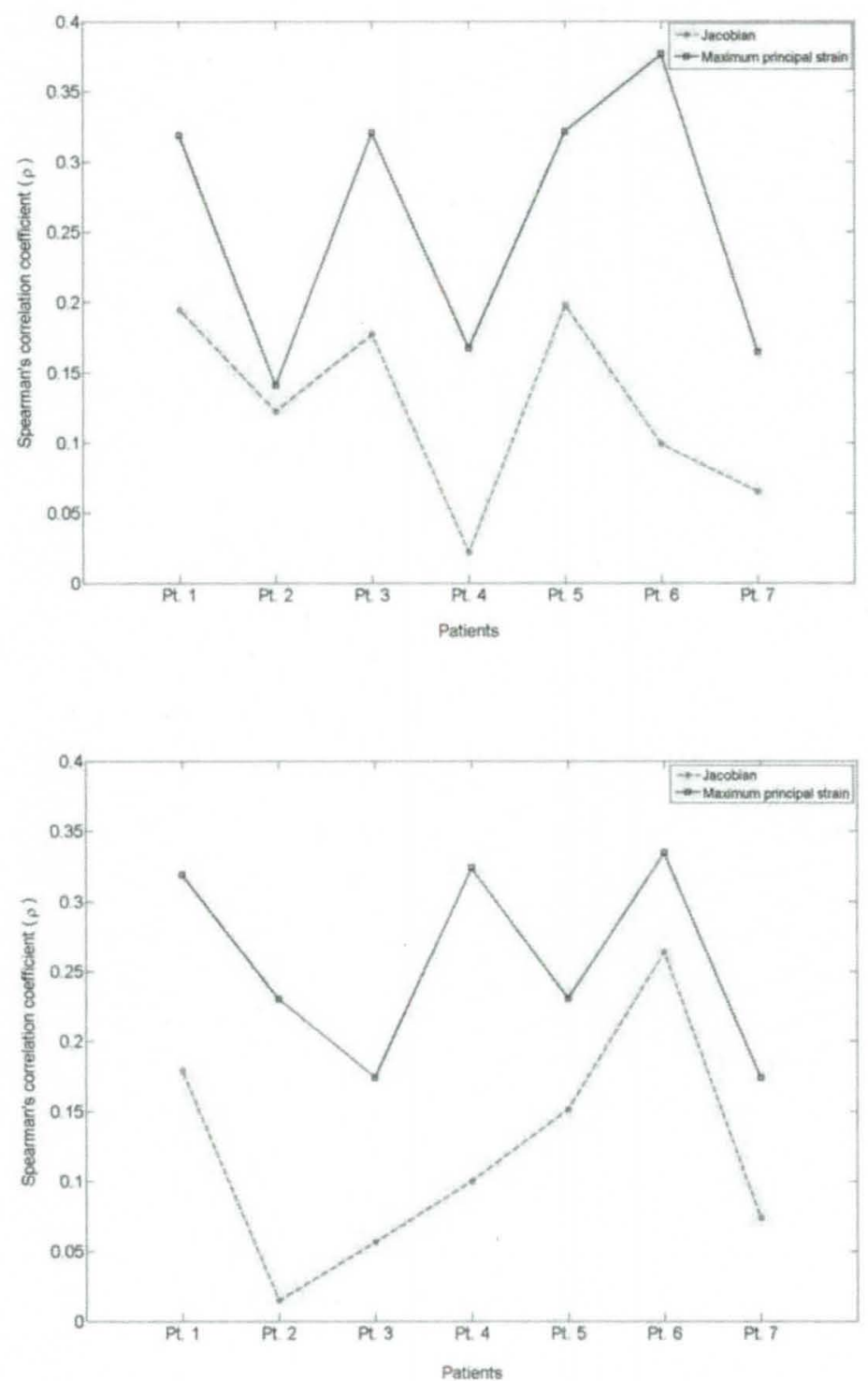

Figure 6.16: The calculated Spearman's correlation coefficient, $\rho$, between Jacobian and maximum principal strain image and SPECT ventilation image, $V_{S P E C T}$, (up) and SPECT perfusion image, $Q_{S P E C T}$, (down) is shown for all patients. 


\section{CHAPTER 7}

\section{CONCLUSIONS AND FUTURE DIRECTIONS}

\subsection{Motivations}

In radiotherapy planning of lung cancer, respiratory motion can be problematic in determination of the target volume, potentially leading to irradiation of risk organs. As a result, nearly all patients who undergo thoracic radiation treatment develop some degree of lung injury and loss of pulmonary function. In modern clinical practice, studies have demonstrated that dose escalations as part of hypofractionated treatments are associated with improved survival rates for NSCLC [85]. Despite this it has also been demonstrated that hypofractionated treatments reduce the level of normal tissue tolerance to radiation and present a higher risk of toxicity.

Prediction and prevention of pulmonary toxicity is of paramount importance in radiation treatment of lung cancer [33] and should involve, optimization of the radiation beam geometry to preferentially pass through dysfunctional regions of the lung and to maximally spare the regions with normal function. The dose volume histograms for the normal and dysfunctional regions of the lung should therefore be calculated separately since the risk of radiation toxicity to the normal and dysfunctional lung need to be considered separately. Incorporation of regional pulmonary function information into the treatment planning process through computation of mechanical strain from 4-D CT will therefore establish a more sophisticated planning practice for the treatment of thoracic malignancies.

\subsection{Contributions}

In this dissertation, we have developed a novel multi-scale optical flow framework with lung-specific assumptions, MOFID, that provides the possibility to enforce physical constraints on the estimated deformation map from 4-D CT thoracic images. MOFID has been extensively validated on eleven 
annotated 4-D CT data sets. It has been shown that use of continuity equation-based mass conservation and gradient constancy as well as use of spatio-temporal smoothness improves prior efforts in measuring and modeling of lung motion from thoracic 4-D CT images.

A new landmark-based elastic registration method to estimate large deformations has also been developed. The proposed method uses a hybrid local/global approach to deformable registration with landmarks providing the local information and mutual information-based registration providing the global statistical intensity information. We have used airway bifurcations as landmarks because they permit accurate point localization and are well-distributed throughout the lungs. These landmarks are extracted from automatically calculated airway centerlines via a path planning method. The proposed elastic registration method has been used in this dissertation in order to register the multi-modality images of the lung (e.g., SPECT and CT). Although we have not registered early and late post-treatment lung CT images with pre-treatment lung CT images (and the corresponding pretreatment/early/late CT-derived indices of lung function), we believe the proposed elastic registration method is well-suited to the task of evaluating the effectiveness of radiation treatment.

We have developed a novel framework for quantification of volumetric deformation maps and mechanical strain from treatment planning 4-D CT images using MOFID. Strain has also been introduced as a novel ventilation metric for assessment of regional lung function from 4-D CT images.

The 4-D CT derived quantitative images of regional lung function and their physiologic accuracy were evaluated on seven non-small cell lung cancer patients and were compared with tomographic SPECT ventilation and perfusion scans in the same patients. In comparison to previous studies, the Jacobian function map derived from MOFID produced a better correlation with both SPECT ventilation and perfusion maps. It was also determined that the maximum principal strain pulmonary function map derived from MOFID outperforms the Jacobian of deformation pulmonary function map which has previously been utilized by several authors as a 4-D CT-derived metric of ventilation.

Having calculated highly accurate spatial deformation maps with MOFID to facilitate regional lung function quantification, our study has provided evidence towards the potential of strain derived from 4-D $\mathrm{CT}$ as a tool in the treatment planning process of thoracic malignancies for functional avoidance and its capability to depict regional pulmonary function. 
The reported correlations between strain derived from 4-D CT with SPECT ventilation and perfusion were found to be significant, validating strain as a measure of regional lung function. We have demonstrated that in the cohort of 7 patients that were studied, the 4-D CT strain measurements were effective in evaluating the functional and dysfunctional lung volume. We hypothesize that assessment of the changes in regional strain post-treatment will permit more effective treatment plans, to minimize damage to the normal lung. We believe this to be especially important for hypofractionated treatment plans where toxicity can be very high.

\subsection{Future Studies}

Current radiation treatment planning techniques are suboptimal and require further optimization by accounting for an accurate regional heterogeneous model of lung function. These findings suggest an image-guided radiation therapy (IGRT) strategy for incorporation of the 4-D CT derived function images into a treatment planning framework for lung cancer patients, in which hypo-functioning regions are identified in order to remove avoidance restriction. This strategy could be combined with function-based conformal avoidance methods. The overarching goal of this study has been to design a planning tool for RT which would be useful for tracking the progression of radiation induced toxicity to surrounding normal tissue during RT, and can be used to design a predictive model of toxicity and to evaluate the effectiveness of treatment.

Limitations of the study reported here include: lack of availability of CT scanners with higher number of detector rows (the patient CT studies were performed on a CT system with 16 detector rows) and lack of availability of a hybrid SPECT/CT system; availability of a hybrid SPECT/CT system would make registration of SPECT with CT unnecessary and should improve on the reported correlation measures.

Additional improvements to physiologic correlation should also be expected through use of Xe-CT or hyperpolarized MR in lieu of ventilation and perfusion SPECT.

We have not studied or compared strain changes from pre-treatment CT studies to early changes after radiation treatment to late changes after radiation treatment. We believe however that the pieces of the project are in place that would permit such comparisons. In each case, SPECT studies should be performed 
to reveal functional and dysfunctional lung volumes and strain should be calculated as an index of lung functionality.

In conjunction with a precise image segmentation algorithm, the proposed functional and biomechanical measurements can be reported on the entire lung, a specific lobe, or sublobar basis, or interpreted relative to the anatomy of other important respiratory structures or risk organs. This will be subject for future studies. 


\section{REFERENCES}

[1] National cancer Institute, VisualsOnline. http://visualsonline.cancer.gov (accessed May 12, 2012).

[2] United States Department of Health and Human Services. Lung cancer distribution in the United States. http://www.hhs.gov/ (accessed May 12, 2012).

[3] Wikipedia, Lung Volumes. http://en.wikipedia.org/wiki/Spirometry (accessed May 12, 2012).

[4] Albert, M. S., Cates, G. D., Driehuys, B., Happer, W., Saam, B., Jr, C. S. S., and Wishnia, A., "Biological magnetic resonance imaging using laser-polarized ${ }^{129} \mathrm{Xe}$," Nature, 370(6486), 199$201,(1994)$.

[5] Alvarez, L., Weickert, J., and S'anchez, J., "Reliable estimation of dense optical flow fields with large displacements," International Journal of Computer Vision, 39(1), 41-56, (2000).

[6] Amini, A. A., Yasheng, C., Curwen, R. W., Mani, V., and Sun, J., "Coupled B-snake grids and constrained thin-plate splines for analysis of 2-D tissue deformations from tagged MRI," IEEE Transactions on Medical Imaging, 17(3), 344-356, (1998).

[7] Baker, S., Scharstein, D., Lewis, J. P., Roth, S., Black, M. J., and Szeliski, R., "A database and evaluation methodology for optical flow," International Journal of Computer Vision, 92(1), 1-31, (2011).

[8] Barron, J. L., Fleet, D. J., and Beauchemin, S. S., "Performance of optical flow techniques," International Journal of Computer Vision (IJCV), 12(1), 43-77, (1994).

[9] Bates, J. H. T., Davis, G. S., Majumdar, A., Butnor, K. J., and Suki, B., "Linking parenchymal disease progression to changes in lung mechanical function by percolation," Am. J. Respir. Crit. Care Med., 176(6), 617-623, (2007).

[10] Beek, E. J. R. V., Wild, J. M., Kauczor, H.-U., Schreiber, W., Mugler, J. P., and Lange, E. E. D., "Functional MRI of the lung using hyperpolarized 3-helium gas," Journal of Magnetic Resonance Imaging, 20(4), 540-554, (2004).

[11] Boldea, V., Sharp, G. C., Jiang, S. B., and Sarrut, D., "4D-CT lung motion estimation with deformable registration: Quantification of motion nonlinearity and hysteresis," Medical Physics, 35(3), 1008-1018, (2008).

[12] Bookstein, F. L., "Principal warps: thin-plate splines and the decomposition of deformations," IEEE Transactions on Pattern Analysis and Machine Intelligence, 11(6), 567-585, (1989).

[13] Brikfellner, W., Figl, M., and Hummel, J.(Eds.), [Applied Medical Image Processing] CRC Press, Taylor and Francis Group, (2011).

[14] Brox, T., Bruhn, A., Papenberg, N., and Weickert, J., "High accuracy optical flow estimation based on a theory of warping," ECCV, 4, 25-36, Czech Republic, (2004).

[15] Bruhn, A., Weickert, J., Feddern, C., Kohlberger, T., and Schnorr, C., "Variational optic flow computation in real-time," IEEE Transactions in Image Processing, 14, 608-615, (2005).

[16] Bruhn, A., Weickert, J., and Schnorr, C., "Lucas/Kanade meets Horn/Schunck: combining local and global optic flow methods," International Journal of Computer Vision, 61(3), 211-231, (2005).

[17] Castillo, E., Castillo, R., Martinez, J., Shenoy, M., and Guerrero, T., "Four-dimensional deformable image registration using trajectory modeling," Physics in Medicine and Biology, $55(1), 305-327,(2010)$. 
[18] Castillo, E., Castillo, R., Zhang, Y., and Guerrero, T., "Compressible image registration for thoracic computed tomography images," Journal of Medical and Biological Engineering, 29(5), 236-247, (2009).

[19] Castillo, R., Castillo, E., Guerra, R., Johnson, V. E., McPhail, T., Garg, A. K., and Guerrero, T., "A framework for evaluation of deformable image registration spatial accuracy using large landmark point sets," Physics in medicine and biology, 54(7), 1849-1870, (2009).

[20] Castillo, R., Castillo, E., Martinez, J., and Guerrero, T., "Ventilation from four-dimensional computed tomography: density versus Jacobian methods," Physics in Medicine and Biology, $55(16), 4661-4685,(2010)$.

[21] Castillo, R., Castillo, E., McCurdy, M., Gomez, D. R., Block, A. M., Bergsma, D., Joy, S., and Guerrero, T., "Spatial correspondence of 4D CT ventilation and SPECT pulmonary perfusion defects in patients with malignant airway stenosis," Physics in Medicine and Biology, 57(7), 1855-1871, (2012).

[22] Chandrashekara, R., Mohiaddin, R. H., and Rueckert, D., "Analysis of 3-D myocardial motion in tagged MR images using nonrigid image registration," IEEE Transactions on Medical Imaging, 23(10), 1245-1250, (2004).

[23] Chon, D., Simon, B. A., Beck, K. C., Shikata, H., Saba, O. I., Won, C., and Hoffman, E. A., "Differences in regional wash-in and wash-out time constants for xenon-CT ventilation studies," Respiratory Physilogy \& Neurobiology, 148(1-2), 65-83, (2005).

[24] Christensen, G. E., and Johnson, H. J., "Consistent image registration," IEEE Transactions on Medical Imaging, 20(7), 568-582, (2001).

[25] Christensen, G. E., Song, J. H., Lu, W., Naqa, I. E., and Low, D. A., "Tracking lung tissue motion and expansion/compression with inverse consistent image registration and spirometry," medical Physics, 34(6), 2155-2163, (2007).

[26] Corpetti, T., Memin, E., and Perez, P., "Dense estimation of fluid flows," IEEE Transactions on Pattern Analysis and Machine Intelligence, 24(3), 365-380, (2002).

[27] Coselmon, M. M., Balter, J. M., Shan, D. L. M., and Kessler., M. L., "Mutual information based CT registration of the lung at exhale and inhale breathing states using thin-plate Splines," Medical Physics, 31(11), 2942-2948, (2004).

[28] Crum, W., Camara, O., Rueckert, D., Bhatia, K., Jenkinson, M., and Hill, D., "Generalised overlap measures for assessment of pairwise and groupwise image registration and segmentation " Medical Imaging Computing and Computer Assisted Intervention (MICCAI), 3749(8), 99-106, (2005).

[29] Davis, M. H., Khotanzad, A., Flamig, D. P., and Harms, S. E., "A physics-based coordinate transformation for 3-D image matching," IEEE Transactions on Medical Imaging, 16(3), 317-328, (1997).

[30] Dawood, M., Buther, F., Xiaoyi, J., and Schafers, K. P., "Respiratory motion correction in 3-D PET data with advanced optical flow algorithms," IEEE Transactions on Medical Imaging, 27(8), 1164-1175, (2008).

[31] Delmarcelle, T., and Hesselink, L., "Visualizing second-order tensor fields with hyperstreamlines," IEEE Computer Graphics and Applications, 13(4), 25-33, (1993).

[32] Devlaminck, V., "A functional for compressible or incompressible elastic deformation estimation," IEEE Signal Processing Letters, 6(7), 162-164, (1999).

[33] Ding, K., Bayouth, J. E., Buatti, J. M., Christensen, G. E., and Reinhardt, J. M., "4DCT-based measurement of changes in pulmonary function following a course of radiation therapy," Medical Physics, 37(3), 1261-1272, (2010). 
[34] Ding, K., Cao, K., Amelon, R. E., Christensen, G. E., Raghavan, M. L., and Reinhardt, J. M., "Comparison of Intensity- and Jacobian-based estimates of lung regional ventilation," Medical Image Analysis for the Clinic: A Grand Challenge, 49-60, (2010).

[35] Ding, K., Cao, K., Christensen, G. E., Hoffman, E. A., and Reinhardt, J. M., "Registration-based regional lung mechanical analysis: Retrospectively reconstructed dynamic imaging versus static breath-hold image acquisition," Proc. SPIE Conf. Medical Imaging, 7262, Lake Buena Vista, FL, (2009).

[36] Ding, K., Yin, Y., Cao, K., Christensen, G. E., Lin, C.-L., Hoffman, E. A., and Reinhardt, J. M., "Evaluation of lobar biomechanics during respiration using image registration," Lecture Notes in Computer Science, Medical Imaging Computing and Computer Assisted Intervention (MICCAI), 12(1), 739-746, London, UK, (2009).

[37] Dougherty, L., Asmuth, J., and Gefter, W., "Alignment of CT lung volumes with an optical flow method," Acad. Radiol., 10(3), 249-254, (2003).

[38] Dougherty, L., Torigian, D., Affusso, J., Asmuth, J., and Gefter, W., "Use of an optical flow method for the analysis of serial CT lung images," Acad. Radiol., 13(1), 14-23, (2006).

[39] Edelman, R. R., Hatabu, H., Tadamura, E., Li, W., and Prasad, P. V., "Noninvasive assessment of regional ventilation in the human lung using oxygen-enhanced magnetic resonance imaging," Nature Medicine, 2(11), 1236-1239, (1996).

[40] Ehrhardt, J., Werner, R., Schmidt-Richberg, A., and Handels, H., "Statistical Modeling of 4D Respiratory Lung Motion Using Diffeomorphic Image Registration," IEEE Transactions on Medical Imaging, 30(2), 251-265, (2011).

[41] Endo, M., Tsunoo, T., Kandatsu, S., Tanada, S., Aradate, H., and Saito, Y., "Four-dimensional computed tomography (4D CT)- concepts and preliminary development," Radiation Medicine, $21(1), 17-22,(2003)$.

[42] Fan, L., and Chen, C. W., "Integrated approach to 3D warping and registration from lung images," SPIE Developments in X-Ray Tomography II, 3772(24-35), Denver, CO, (1999).

[43] Fan, L., Chen, C. W., Reinhardt, J. M., and Hoffman, E. A., "Evaluation and application of 3D lung warping and registration model using HRCT images," SPIE Conf. Medical Imaging, 4321, 234-243, San Diego, CA, (2001).

[44] Fitzpatrick, J. M., "A method for calculating velocity in time dependent images based on the continuity equation," Computer Vision Pattern Recognition, 78-81, (1985).

[45] Fitzpatrick, J. M., and West, J. B., "The distribution of target registration error in rigid-body pointbased registration," IEEE Transactions on Medical Imaging, 20(9), 917-927, (2001).

[46] Ford, E. C., Mageras, G. S., Yorke, E., and Ling, C. C., "Respiration-correlated spiral CT: a method of measuring respiratory-induced anatomic motion for radiation treatment planning," Medical Physics, 30(1), 88-97, (2003).

[47] Gee, J., Reivich, M., and Bajcsy, R., "Elastically deforming 3D atlas to match anatomical brain images," J Comput Assist Tomogr, 17(2), 225-236, (1993).

[48] Gennert, M. A., and Negahdaripour, S., [Relaxing the brightness constancy assumption in computer optical flow], (1987).

[49] Glenny, R. W., "Teaching ventilation/perfusion relationships in the lung," Advances in Physiology Education, 32(3), 192-195, (2008).

[50] Gorbunova, V., Durrleman, S., Lo, P., Pennec, X., and de Bruijne, M., "Curve- and Surface-based registration of lung CT images via currents," Second International Workshop on Pulmonary Image Analysis, 15-25, (2009). 
[51] Gorbunova, V., Lo, P., Ashraf, H., Dirksen, A., Nielsen, M., and de Bruijne, M., "Weight preserving image registration for monitoring disease progression in lung $\mathrm{CT}$ medical image computing and computer-assisted intervention," Medical Imaging Computing and Computer Assisted Intervention (MICCAI), 5242, 863-870, (2008).

[52] Gorbunova, V., Sporring, J., Lo, P., Dirksen, A., and Bruijne, M. d., "Mass preserving image registration: Results of evaluation of methods for pulmonary image registration 2010 challenge," Medical Image Analysis for the Clinic: A Grand Challenge, 155-164, (2010).

[53] Greaves, I., and Colebatch, H., "Elastic behavior and structure of normal and emphysematous lungs post mortem," Am Rev Respir Dis, 121(1), 127-136, (1980).

[54] Guenther, D., Hanisch, G., and Kauczor, H., "Functional MR imaging of pulmonary ventilation using hyperpolarized noble gases," Acta Radiolog., 41(6), 519-128, (2000).

[55] Guerrero, T., G.Zhang, Huang, T., and Lin, K., "Intrathoracic tumour motion estimation from CT imaging using the 3D optical flow method," Physics in Medicine and Biology, 49, 4147-4161, (2004).

[56] Guerrero, T., Sanders, K., Castillo, E., Zhang, Y., Bidaut, L., Pan, T., and Komaki1, R., "Dynamic ventilation imaging from four-dimensional computed tomography," Physics in Medicine and Biology, 51(4), 777-791, (2006).

[57] Guerrero, T., Sanders, K., Noyola-Martinez, J., Castillo, E., Zhang, Y. et al., "Quantification of regional ventilation from treatment planning CT," Int J Radiat Oncol Biol Phys, 62(3), 630-634, (2005).

[58] Gur, D., Drayer, B., Borovetz, H., Griffith, B., Hardesty, R., and Wolfson, S., "Dynamic computed tomography of the lung: regional ventilation measurements," J Comput Assist Tomogr, 3(6), 749-753, (1979).

[59] Harris, R. S., and Schuster, D. P., "Visualizing lung function with positron emission tomography" Journal of Applied Physiology, 102(1), 448-458, (2007).

[60] Haussecker, H. W., and Fleet, D. J., "Computing optical flow with physical models of brightness variation," IEEE Transactions on Pattern Analysis and Machine Intelligence, 23(6), 661-673, (2001).

[61] Heath, E., Collins, D. L., Keall, P. J., Dong, L., and Seuntjens, J., "Quantification of accuracy of the automated nonlinear image matching and anatomical labeling (ANIMAL) nonlinear registration algorithm for 4D CT images of lung," Medical Physics, 34(11), 4409-4421, (2007).

[62] Hill, D., Batchelor, P., Holden, M., and Hawkes, D., "Medical image registration " Physics in Medicine and Biology, 46(3), R1-R45, (2001).

[63] Hilsmann, A., Vik, T., Kaus, M., Franks, K., Bissonette, J.-P., Purdie, T., Beziak, A., and Aach, T., "Deformable 4DCT lung registration with vessel bifurcations," International Conference of Computer Assisted Radiology and Surgery (CARS), (2007).

[64] Hoffman, E. A., and Chon, D., "Computed tomography studies of lung ventilation and perfusion," Proc Am Thorac Soc, 2(6), 492-498, (2005).

[65] Hoffman, E. A., Sinak, L. J., Robb, R. A., and Ritman, E. L., "Noninvasive quantitative imaging of shape and volume of lungs," Journal of Applied Physiology, 54(5), 1414-1421, (1983).

[66] Horn, B. K. P., and Schunck, B. G., "Determining optical flow," Artificial Intelligence, 17, 185203, (1981).

[67] Hsieh, J.(Eds.), [Computed Tomography: Principles, Design, Artifacts, and Recent Advances] SPIE Press, (2003). 
[68] Hua-Mei, C., Varshney, P. K., and Arora, M. K., "Performance of mutual information similarity measure for registration of multitemporal remote sensing images," IEEE Transactions on Geoscience and Remote Sensing, 41(11), 2445-2454, (2003).

[69] Huang, J., Abendschein, D., Davila-Roman, V. G., and Amini, A. A., "Spatio-temporal tracking of myocardial deformations with a 4-D B-spline model from tagged MRI," IEEE Transactions on Medical Imaging, 18(10), 957-972, (1999).

[70] Hubmayr, R. D., Rodarte, J. R., Walters, B. J., and Tonelli, F. M., "Regional ventilation during spontaneous breathing and mechanical ventilation in dogs," Journal of Applied Physiology, 63(6), 2467-2475, (1987).

[71] Ibanez, L., Schroeder, W., Ng, L., and Cates, J.(Eds.), [The ITK software guide] Kitware Inc., second edition, (2005).

[72] Ireland, R., Bragg, C., McJury, M., Woodhouse, N., Fichele, S., van Beek, E., Wild, J., and Hatton, M., "Feasibility of image registration and intensity-modulated radiotherapy planning with hyperpolarized helium-3 magnetic resonance imaging for non-small-cell lung cancer," Int J Radiat Oncol Biol Phys, 68(1), 273-281, (2007).

[73] Jannin, P., Fitzpatrick, J. M., Hawkes, D. J., Pennec, X., Shahidl, R., and Vannier, M. W., "Validation of medical image processing in image-guided therapy," IEEE Transactions on Medical Imaging, 21(12), 1445-1449, (2002).

[74] Johnson, H. J., and Christensen, G. E., "Consistent landmark and intensity-based image registration," IEEE Transactions on Medical Imaging, 21(5), 450-461, (2002).

[75] Joshi, S. C., Miller, M. I., Christensen, G. E., Banerjee, A., Coogan, T. A. et al., "Hierarchical brain mapping via a generalized Dirichlet solution for mapping brain manifolds," SPIE Conf. Medical Imaging, 2573, 278-289, (1995).

[76] Kabus, S., Klinder, T., Murphy, K., van Ginneken, B., van Lorenz, C., and Pluim, J. P. W., "Evaluation of 4D-CT lung registration," Medical Imaging Computing and Computer Assisted Intervention (MICCAI), 12(1), 747-754, (2009).

[77] Kabus, S., and Lorenz, C., "Fast elastic image registration," Medical Image Analysis for the Clinic: A Grand Challenge, 81-89, (2010).

[78] Kaus, M., Netsch, T., Kabus, S., Pekar, V., McNutt, T., and Fischer, B., "Estimation of organ motion from 4D CT for 4D radiation therapy planning of lung cancer," Lecture Notes in Computer Science, Medical Imaging Computing and Computer Assisted Intervention (MICCAI), 3217, 1017-1024, (2004).

[79] Kavanagh, B. D., and Timmerman, R. D., "Stereotactic radiosurgery and stereotactic body radiation therapy: an overview of technical considerations and clinical applications," Hematology/oncology clinics of North America, 20(1), 87-95, (2006).

[80] Keall, P., "Four-dimensional computed tomography imaging and treatment planning," Seminars in Radiation Oncology, 14(1), 81-90, (2004).

[81] Keall, P. J., Starkschall, G., Shukla, H., Forster, K. M., Ortiz, V. et al., "Acquiring 4D thoracic CT scans using a multislice helical method," Physics in Medicine and Biology, 49(10), 2053-2068, (2004).

[82] Kindlmann, G., "Superquadric Tensor Glyphs," Proceedings of IEEE TVCG/EG Symposium on Visualization, 147-154, (2004).

[83] Klein, S., and Staring, M.(Eds.), [The manual of Elastix], (2010).

[84] Klein, S., Staring, M., Murphy, K., Viergever, M. A., and Pluim, J., "elastix: A toolbox for intensity-based medical image registration," IEEE Transactions on Medical Imaging, 29(1), 196$205,(2010)$. 
[85] Kong, F.-M., Haken, R. K. T., Schipper, M. J., Sullivan, M. A., Chen, M., Lopez, C., Kalemkerian, G. P., and Hayman, J. A., "High-dose radiation improved local tumor control and overall survival in patients with inoperable/unresectable non-small-cell lung cancer: long-term results of a radiation dose escalation study," Int J Radiat Oncol Biol Phys, 63(2), 324-333, (2005).

[86] Kreck, T. C., Krueger, M. A., Altemeier, W. A., Sinclair, S. E., Robertson, H. T. et al., "Determination of regional ventilation and perfusion in the lung using xenon and computed tomography," Journal of Applied Physiology, 91(4), 1741-1749, (2001).

[87] Kybic, J., and Unser, M., "Fast parametric elastic image registration," IEEE Transactions on Image Processing, 12(11), 1427-1442, (2003).

[88] Li, B., Christensen, G., Hoffman, E., McLennan, G., and Reinhardt, J., "Pulmonary CT image registration and warping for tracking tissue deformation during the respiratory cycle through $3 \mathrm{D}$ consistent image registration," Medical Physics, 35(12), 5575-5583, (2008).

[89] Li, B., Christensen, G. E., McLennan, G., Hoffman, E. A., and Reinhardt, J. M., "Establishing a normative atlas of the human lung: Inter-subject warping and registration of volumetric CT images," Acad. Radiol., 10(3), 255-265, (2003).

[90] Li, T., Schreibmann, E., Yang, Y., and Xing, L., "Motion correction for improved target localization with on-board cone-beam computed tomography," Physics in Medicine and Biology, 51(2), 253-268, (2006).

[91] Liu, H., Hong, T.-H., Herman, M., Camus, T., and Chellappa, R., "Accuracy vs efficiency tradeoffs in optical flow algorithms," Computer Vision and Image Understanding, 72(3), 271-286, (1998).

[92] Loeckx, D., Smeets, D., Keustermans, J., Hermans, J., Maes, F., Vandermeulen, D., and Suetens, P., "3D lung registration using splineMIRIT and robust tree registration (RTR)," Medical Image Analysis for the Clinic: A Grand Challenge, 109-117, (2010).

[93] Low, D., Nystrom, M., Kalinin, E., Parikh, P., Dempsey, J. et al., "A method for the reconstruction of four-dimensional synchronized CT scans acquired during free breathing," Medical Physics, 30(6), 1254-1263, (2003).

[94] Lu, W., Chen, M.-L., Olivera, G. H., Ruchala, K. J., and Mackie, T. R., "Fast free-form deformable registration via calculus of variations," Physics in medicine and biology, 49(14), 30673087, (2004).

[95] Lu , W., Parikh, P., El Naqa, I., Nystrom, M., Hubenschmidt, J. et al., "Quantitation of the reconstruction quality of a four-dimensional computed tomography process for lung cancer patients," Medical Physics, 32(4), 890-901, (2005).

[96] Lucas, B. D., and Kanade, T., "An iterative image registration technique with an application to stereo vision," Proceedings of DARPA Image Understanding workshop, 121-130, (1981).

[97] Mageras, G. S., Pevsner, A., Yorke, E. D., Rosenzweig, K. E., Ford, E. C. et al., "Measurement of lung tumor motion using respiration-correlated CT," International journal of radiation oncology, biology, physics, 60(3), 933-941, (2004).

[98] Maintz, J. B. A., and Viergever, M. A., "A survey of medical image registration," Medical Image Analysis, 2(2), 1-36, (1997).

[99] Marcucci, C., Nyhan, D., and Simon, B., "Distribution of pulmonary ventilation using Xeenhanced computed tomography in prone and supine dogs," Journal of Applied Physiology, 90(2), 421-430, (2001).

[100] Massaro, D., Clerch, L., Temple, D., and Baier, H., "Surfactant deficiency in rats without a decreased amount of extracellular surfactant," J Clin Invest, 71(6), 1536-1543, (1983). 
[101] Matsopoulos, G. K., Mouravliansky, N. A., Asvestas, P. A., Delibasis, K. K., and Kouloulias, V., "Thoracic non-rigid registration combining self-organizing maps and radial basis functions," Medical Image Analysis, 9(3), 237-254, (2005).

[102] Mayo, J. R., Aldrich, J., and Müller, N. L., "Radiation exposure at chest CT: A statement of the Fleischner society," Radiology, 228(1), 15-21, (2003).

[103] McCane, B., Novins, K., Crannitch, D., and Galvin, B., "On benchmarking optical flow," Computer Vision and Image Understanding, 84(1), 126-143, (2001).

[104] McClelland, J. R., Blackall, J. M., Tarte, S., Chandler, A. C., Hughes, S., Ahmad, S., Landau, D. B., and Hawkes, D. J., "A continuous 4D motion model from multiple respiratory cycles for use in lung radiotherapy " Medical Physics, 33(9), 3348-3358, (2006).

[105] Metz, C. T., Klein, S., Schaap, M., van Walsum, T., and Niessen, W. J., "Nonrigid registration of dynamic medical imaging data using $\mathrm{nD}+\mathrm{t} \mathrm{B}$-splines and a groupwise optimization approach," Medical Image Analysis, 15(2), 238-249, (2011).

[106] Modat, M., McClelland, J., and Ourselin, S., "Lung registration using the NiftyReg package," Medical Image Analysis for the Clinic: A Grand Challenge, 33-42, (2010).

[107] Modersitzki, J.(Eds.), [Numerical methods for image registration] Oxford University Press, New York, (2004).

[108] Murphy, K., van Ginneken, B., Reinhardt, J., Kabus, S., Ding, K. et al., "Evaluation of registration methods on thoracic CT: The EMPIRE10 challenge," IEEE Transactions on Medical Imaging, 30(11), 1901-1920, (2011).

[109] Musch, G., Layfield, J. D. H., Harris, R. S., Melo, M. F. V., Winkler, T., Callahan, R. J., Fischman, A. J., and Venegas, J. G., "Topographical distribution of pulmonary perfusion and ventilation, assessed by PET in supine and prone humans," Journal of applied physiology, 93(5), 1841-1851, (2002).

[110] Nagel, H.-H., and Enkelmann, W., "An investigation of smoothness constraints for the estimation of displacement vector fields from image sequences," IEEE Transactions on Pattern Analysis and Machine Intelligence, 8(5), 565-593, (1986).

[111] Narabayashi, 1., Suematsu, T., and Suematsu, C., "Assessment of SPECT ventilation-perfusion imaging in patients with lung cancer," European journal of nuclear medicine, 12(7), 329-332, (1986).

[112] Negahdar, M., Ahmadian, A., and Navab, N., "Stiff and efficient middle axis extraction of airways for virtual bronchoscopy," International Conference of Telemedicine and e-Health, Tehran, Iran, (2006).

[113] Negahdar, M., Ahmadian, A., Navab, N., and Firouznia, K., "Automated path planning algorithm for virtual bronchoscopy," Iranian Journal of Medical Physics (IJMP), 3(11), 51-62, (2006).

[114] Negahdar, M., Ahmadian, A., Navab, N., and Firouznia, K., "Path planning for virtual bronchoscopy," 28th Annual International Conference of the IEEE Engineering in Medicine and Biology Society, 2006. EMBS '06., 156-159, (2006).

[115] Negahdar, M., and Amini, A. A., "Tracking planar lung motion in 4D CT with optical flow: validations and comparison of global, local, and local-global methods," SPIE Conf. on Biomedical Applications in Molecular, Structural and Functional Imaging, 7626-74, San Diego, CA, USA (2010).

[116] Negahdar, M., and Amini, A. A., "Estimation of affine motion from projection data using a mass conservation model," 33rd Annual International Conference of the IEEE Engineering in Medicine and Biology Society, 2011. EMBS '11, 8041-8044, Boston, MA, USA, (2011). 
[117] Negahdar, M., and Amini, A. A., "Multi-scale optical flow including normalized mutual information for planar deformable lung motion estimation from 4D CT," 33rd Annual International Conference of the IEEE Engineering in Medicine and Biology Society, 2011. EMBS '11, 4888-4892, Boston, MA, USA, (2011).

[118] Negahdar, M., and Amini, A. A., "Planar deformable motion estimation incorporating mass conservation and image gradient constancy," IEEE International Symposium on Biomedical Imaging (ISBI): From Nano to Macro, 536-540, Chicago, IL, USA, (2011).

[119] Negahdar, M., and Amini, A. A., "A 3-D optical flow technique based on mass conservation for deformable motion estimation from 4-D CT images of the lung," SPIE Conf. on Biomedical Applications in Molecular, Structural and Functional Imaging, 8317-50, San Diego, CA, USA (2012).

[120] Negahdar, M., and Amini, A. A., "A multi-scale optical flow technique incorporating mass conservation for deformable motion estimation (MOFID) from 4-D X-ray CT images of the lung," IEEE Transactions on Medical Imaging, [under review], (2012).

[121] Negahdar, M., and Amini, A. A., "A novel optical flow method for myocardial deformation analysis from tagged MRI," SCMR-ISMRM Workshop on Exploring New Dimensions of cardiovascular Flow and Motion, Orlando, FL, USA, (2012).

[122] Negahdar, M., and Amini, A. A., "Regional lung strains via a volumetric mass conserving optical flow model," IEEE International Symposium on Biomedical Imaging (ISBI): From Nano to Macro, Barcelona, Spain, (2012).

[123] Negahdar, M., Amini, A. A., Zacarias, A., Lynch, B. L., Milam, R. A., Woo, S. Y., and Dunlap, N., "Strain as a novel index of regional function from treatment planning thoracic 4-D CT," Journal of Thoracic Oncology, supplement for the 2012 Chicago Multidisciplinary Symposium in Thoracic Oncology, (2012).

[124] Negahdar, M., Dunlap, N., Zacarias, A., Civelek, A. C., Woo, S. Y., and Amini, A. A., "Strain as a novel index of regional pulmonary function from thoracic 4-D CT: In-vivo validation with tomographic (SPECT) ventilation and perfusion," Medical Physics, [under review], (2012).

[125] Negahdar, M., Zacarias, A., Dunlap, N., Woo, S. Y., and Amini, A. A., "An automated landmarkbased elastic registration technique for large deformation recovery from 4-D CT lung images," SPIE Conf. on Biomedical Applications in Molecular, Structural and Functional Imaging, 831751, San Diego, CA, USA (2012).

[126] Pan, T., "Comparison of helical and cine acquisitions for 4D-CT imaging with multislice CT," Medical Physics, 32(2), 627-634, (2005).

[127] Pan, T., Lee, T.-Y., Rietzel, E., and Chen, G. T. Y., "4D-CT imaging of a volume influenced by respiratory motion on multi-slice CT," Medical physics, 31(2), 333-340, (2004).

[128] Papp, L., Zuhayra, M., Henze, E., and Luetzen, U., "Extended normalized mutual information for lung SPECT-CT registration," Bioinformatics and Biomedical Engineering, 2009. ICBBE 2009. 3rd International Conference on, 1-3, (2009).

[129] Pekar, V., Glailin, E., and Rohr, K., "An adaptive irregular grid approach for 3D deformable image registration," Physics in medicine and biology, 51(2), 361-377, (2006).

[130] Periaswamy, S., and Farid, H., "Elastic registration in the presence of intensity variations," IEEE Transactions on Medical Imaging, 22(7), 865-874, (2003).

[131] Pevsner, A., Davis, B., Joshi, S., Hertanto, A., Mechalakos, J. et al., "Evaluation of an automated deformable image matching method for quantifying lung motion in respiration-correlated CT images," Medical Physics, 33(2), 369-376, (2006). 
[132] Plathow, C., Schoebinger, M., Herth, F., Tuengerthal, S., Meinzer, H., and Kauczor, H., "Estimation of pulmonary motion in healthy subjects and patients with intrathoracic tumors using 3D-dynamic MRI: initial results," Korean J Radiol., 10(6), 559-567, (2009).

[133] Press, W. H., Flannery, B. P., Teukolsky, S. A., and Vetterling, W. T.(Eds.), [Numerical Recipes in C: The Art of Scientific Computing] Cambridge University Press, (1988).

[134] Qiu, M., "Computing optical flow based on the mass-conserving assumption," International Conference on Pattern Recognition, 3(3), 7041-7044, (2000).

[135] Radeva, P., Amini, A. A., and Huang, J., "Deformable B-solids and implicit snakes for 3D localization and tracking of SPAMM MRI data," Computer Vision and Image Understanding, 66(2), 163-178, (1997).

[136] Reinhardt, J. M., Christensen, G. E., Hoffman, E. A., Ding, K., and Cao, K.(Eds.), [Registrationderived estimates of local lung expansion as surrogates for regional ventilation] Springer Berlin/Heidelberg, Kerkrade, The Netherlands, 763-774, (2007).

[137] Reinhardt, J. M., Ding, K., Cao, K., Christensen, G. E., Hoffman, E. A., and Bodas, S. V., "Registration-based estimates of local lung tissue expansion compared to xenon-CT measures of specific ventilation," Medical Image Analysis, 12(6), 752-763, (2008).

[138] Rietzel, E., and Chen, G., "Deformable registration of 4D computed tomography data," Medical Physics, 33(11), 4423-4430, (2006).

[139] Rietzel, E., Pan, T., and Chen, G., "Four-dimensional computed tomography: Image formation and clinical protocol," Medical Physics, 32(4), 974-989, (2005).

[140] Ritman, E. L., Kinsey, J. H., Robb, R. A., Gilbert, B. K., Harris, L. D., and Wood, E. H., "Threedimensional imaging of heart, lungs, and circulation," Science, 210(4467), 273-280, (1980).

[141] Robb, R. A., Hoffman, E. A., Sinak, L. J., Harris, L. D., and Ritman, E. L., "High-speed threedimensional X-ray computed tomography: The dynamic spatial reconstructor," Proceedings of the IEEE, 71(3), 308-319, (1983).

[142] Rodarte, J. R., Hubmayr, R. D., Stamenovic, D., and Walters, B. J., "Regional lung strain in dogs during deflation from total lung capacity," Journal of Applied Physiology, 58(1), 164-172, (1985).

[143] Rohr, K., Stiehl, H. S., Sprengel, R., Buzug, T. M., Weese, J., and Kuhn, M. H., "Landmark-based elastic registration using approximating thin-plate splines," IEEE Transactions on Medical Imaging, 20(6), 526-534, (2001).

[144] Rueckert, D., Sonoda, L. I., Hayes, C., Hill, D. L. G., Leach, M. O., and Hawkes, D. J., "Nonrigid registration using free-form deformations: application to breast MR images," IEEE Transactions on Medical Imaging, 18(8), 712-721, (1999).

[145] Santyr, G. E., Couch, M. J., Lam, W. W., Ouriadov, A., Drangova, M., McCormack, D. G., and Holdsworth, D. W., "Comparison of hyperpolarized ${ }^{3} \mathrm{He}$ MRI with Xe-enhanced computed tomography imaging for ventilation mapping of rat lung," NMR in Biomedicine, 24(19), 1073$1080,(2011)$.

[146] Sarrut, D., Boldae, V., Miguet, S., and Ginestet, C., "Simulation of 4-D CT images from deformable registration between inhale and exhale breath-hold CT scans," Medical Physics, 33(3), 605-617, (2006).

[147] Schmidt-Richberg, A., Ehrhardt, J., Werner, R., and Handels, H., "Diffeomorphic diffusion registration of lung CT images," Medical Image Analysis for the Clinic: A Grand Challenge, 55$62,(2010)$.

[148] Sluimer, I., Schilham, A., Prokop, M., and van Ginneken, B., "Computer analysis of computed tomography scans of the lung: a survey," IEEE Transactions on Medical Imaging, 25(4), 385-405, (2006). 
[149] American Cancer Society, [Cancer Facts and Figures], (2012).

[150] Song Chun, Z., and Yuille, A., "Region competition: unifying snakes, region growing, and Bayes/MDL for multiband image segmentation," IEEE Transactions on Pattern Analysis and Machine Intelligence, 18(9), 884-900, (1996).

[151] Song, G., Tustison, N., Avants, B., and Gee, J. C., "Lung CT image registration using diffeomorphic transformation models," Medical Image Analysis for the Clinic: A Grand Challenge, 23-32, (2010).

[152] Song, S. M., and Leahy, R. M., "Computation of 3-D velocity fields from 3-D cine CT images of a human heart," IEEE Transactions on Medical Imaging, 10(3), 295-306, (1991).

[153] Sonke, J., Zijp, L., Remeijer, P., and van Herk, M., "Respiratory correlated cone beam CT," Medical Physics, 32(4), 1176-1186, (2005).

[154] Staring, M., Klein, S., and Pluim, J. P. W., "A rigidity penalty term for nonrigid registration," Medical Physics, 34, 4098-4108, (2007).

[155] Staring, M., Klein, S., Reiber, J. H. C., Niessen, W. J., and Stoel, B. C., "Pulmonary image registration with elastix using a standard intensity-based algorithm," Medical Image Analysis for the Clinic: A Grand Challenge, 73-79, (2010).

[156] Stewart, C., Lee, Y., and Tsai, C., "An uncertainty-driven hybrid of intensity-based and featurebased registration with application to retinal and lung CT images," Medical Imaging Computing and Computer Assisted Intervention (MICCAI), 3217, 870-877, (2004).

[157] Studholme, C., Hill, D. L. G., and Hawkes, D. J., "An overlap invariant entropy measure of 3D medical image alignment," Pattern Recognition, 32(1), 71-86, (1999).

[158] Suga, K., "Technical and analytical advances in pulmonary ventilation SPECT with xenon-133 gas and Tc-99m-Technegas," Annals of Nuclear Medicine, 16(5), 303-310, (2002).

[159] Suki, B., and Bates, J. H. T., "Lung tissue mechanics as an emergent phenomenon," Journal of Applied Physiology, 110(4), 1111-1118, (2011).

[160] Sundaram, T. A., and Gee, J. C., "Towards a model of lung biomechanics: pulmonary kinematics via registration of serial lung images.," Medical Image Analysis, 9(6), 524-537, (2005).

[161] Szeliski, R., and Coughlan, J., "Spline-based image registration," International Journal of Computer Vision, 22(3), 199-218, (1997).

[162] Tawhai, M. H., and Lin, C. L., "Image-based modeling of lung structure and function," J Magn Reson Imaging, 32(6), 1421-1431, (2010).

[163] Teng, C.-H., Lai, S.-H., Chen, Y.-S., and Hsu, W.-H., "Accurate optical flow computation under non-uniform brightness variations," Computer Vision and Image Understanding, 97(3), 315-346, (2005).

[164] Torigian, D. A., Gefter, W. B., Affuso, J. D., Emami, K., and Dougherty, L., "Application of an optical flow method to inspiratory and expiratory lung MDCT to assess regional air trapping: A feasibility study" American Journal of Roentgenology, 188(3), 276-280, (2007).

[165] Tustison, N., [Biventricular myocardial strains with anatomical NURBS models from tagged MRI], Ph.D. Thesis, Washington University, (2004).

[166] Tustison, N. J., Avants, B. B., and Gee, J. C., "Directly manipulated free-form deformation image registration," IEEE Transactions on Image Processing, 18(3), 624-635, (2009).

[167] Tustison, N. J., Cook, T. S., Song, G., and Gee, J. C., "Pulmonary kinematics from image data: A review," Academic Radiology, 18(4), 402-417, (2011).

[168] Underberg, R. W. M., Lagerwaard, F. J., Cuijpers, J. P., Stolman, B. J., Koste, J. R. v. S. d., and Senan, S., "Four-dimensional CT scans for treatment planning in stereotactic radiotherapy for 
stage I lung cancer," International journal of radiation oncology, biology, physics, 60(4), 12831290, (2004).

[169] Unser, M., "Splines: a perfect fit for signal and image processing," IEEE Signal Processing Magazine, 16(6), 22-38, (1999).

[170] Uppaluri, R., Hoffman, E. A., Sonka, M., Hartley, P. G., Hunninghake, G. W., and McLennan, G., "Computer recognition of regional lung disease patterns " Am. J. Respir. Crit. Care Med., 160(2), 648-654, (1999).

[171] Urschler, M., and Bischof, H., "Assessing breathing motion by shape matching of lung and diaphragm surfaces," SPIE Int. Opt. Eng., 5746, 440-452, (2005).

[172] Vandemeulebroucke, J., Rit, S., Schaerer, J., and Sarrut, D., "Deformable image registration with automated motion-mask extraction," Medical Image Analysis for the Clinic: A Grand Challenge, $119-125,(2010)$.

[173] Vandemeulebroucke, J., Sarrut, D., and Clarysse, P., "The POPI-model, a point-validated pixelbased breathing thorax model," International Conference on the Use of Computers in Radiation Therapy (ICCR), Toronto, Canada, (2007).

[174] Vedam, S. S., Keall, P. J., Kini, V. R., Mostafavi, H., Shukla, H. P., and Mohan, R., "Acquiring a four-dimensional computed tomography dataset using an external respiratory signal," Physics in medicine and biology, 48(1), 45-62, (2003).

[175] Voorhees, A., An, J., Berger, K. I., Goldring, R. M., and Chen, Q., "Magnetic resonance imagingbased spirometry for regional assessment of pulmonary function," Magnetic Resonance in Medicine, 54(5), 1146-1154, (2005).

[176] Wang, G., Yu, H., and Man, B. D., "An outlook on x-ray CT research and development," Medical Physics, 35(3), 1051-1064, (2008).

[177] Wang, H., Dong, L., O'Daniel, J., Mohan, R., Garden, A. S. et al., "Validation of an accelerated 'demons' algorithm for deformable image registration in radiation therapy," Physics in Medicine and Biology, 50, 2887-2905, (2005).

[178] Wei, C., Song, Z., Correia, S., and Tate, D. F., "Visualizing diffusion tensor imaging data with merging ellipsoids," IEEE Pacific Visualization Symposium, 2009. PacificVis '09., 145-151, (2009).

[179] Weibel, E. R., "What makes a good lung?," Swiss medical weekly, 139(27-28), 375-386, (2009).

[180] Weickert, J., Bruhn, A., Brox, T., and Papenberg, N.. [A Survey on variational optic flow methods for small displacements] Springer, Berlin, (2006).

[181] Weickert, J., and Schnorr, C., "Variational optic flow computation with a spatio-temporal smoothness constraint," J. Math. Imaging and Vision, 14(3), 245-255, (2001).

[182] Wellman, T. J., Winkler, T., Costa, E. L. V., Musch, G., Harris, R. S., Venegas, J. G., and Melo, M. F. V., "Measurement of regional specific lung volume change using respiratory-gated PET of inhaled ${ }^{13} \mathrm{~N}$-Nitrogen," Journal of Nucl Med., 51(4), 646-653, (2010).

[183] Weruaga, L., Morales, J., Nunez, L., and Verdu, R., "Estimating volumetric motion in human thorax with parametric matching constraints," IEEE Transactions on Medical Imaging, 22(6), 766772, (2003).

[184] Wildes, R. P., Amabile, M. J., Lanzillotto, A.-M., and Leu, T.-S., "Recovering estimates of fluid flow from image sequence data," Computer Vision and Image Understanding, 80(2), 246-266, (2000).

[185] Woods, R. P., I. Bankman (Eds.), [Handbook of Medical Image Processing and Analysis] Academic Press, Inc., Orlando, USA, 2nd Edition, 569-575, (2009). 
[186] Wörz, S., and Rohr, K., "Physics-based elastic registration using non-radial basis functions and including landmark localization uncertainties," Computer Vision and Image Understanding, 111(3), 263-274, (2008).

[187] Wu, Z., Rietzel, E., Boldea, V., Sarrut, D., and Sharp, G. C., "Evaluation of deformable registration of patient lung 4DCT with subanatomical region segmentations," Medical Physics, $35(2), 775-781,(2008)$.

[188] Wünsche, B., "The Visualization of 3D Stress and Strain Tensor Fields," Proceedings of the 3rd New Zealand Computer Science Research Student Conference, 109-116, University of Waikato, Hamilton, New Zealand, (1999).

[189] Yamamoto, T., Kabus, S., Berg, J. v., Lorenz, C., Goris, M., Loo, B., and Keall, P., "Evaluation of four-dimensional (4D) computed tomography (CT) pulmonary ventilation imaging by comparison with single photon emission computed tomography (SPECT) scans for a lung cancer patient," Third Int. Workshop on Pulmonary Image Analysis, MICCAI 117-128, (2010).

[190] Yamamoto, T., Kabus, S., Klinder, T., Berg, J. v., Lorenz, C., Loo, B. W. J., and Keall, P. J., "Four-dimensional computed tomography pulmonary ventilation images vary with deformable image registration algorithms and metrics," Medical Physics, 38(3), 1348-1358, (2011).

[191] Yamamoto, T., Kabus, S., von Berg, J., Lorenz , C., and Keall, P., "Impact of four-dimensional computed tomography pulmonary ventilation imaging-based functional avoidance for lung cancer radiotherapy," Int J Radiat Oncol Biol Phys, 79(1), 279-288, (2011).

[192] Yamamura, J., Tornquist, K., Buchert, R., Wildberger, J., Nagel, H. D., Dichtl, D., Adam, G., and Wedegärtner, U., "Simulated low-dose computed tomography in oncological patients: A feasibility study," Journal of Computer Assisted Tomography, 34(2), 302-308, (2010).

[193] Yang, D., Lu, W., Low, D. A., Deasy, J. O., Hope, A. J., and Naqa, I. E., "4D-CT motion estimation using deformable image registration and 5D respiratory motion modeling," Medical Physics, 35(10), 4577-4590, (2008).

[194] Yaremko, B. P., Guerrero, T. M., Noyola-Martinez, J., Guerra, R., Lege, D. G. et al., "Reduction of normal lung irradiation in locally advanced non-small-cell lung cancer patients, using ventilation images for functional avoidance," Infect Immun, 68(2), 562-571, (2007).

[195] Yernault, J., Jonghe, M. d., Coster, A. d., and Englert, M., "Pulmonary mechanics in diffuse fibrosing alveolitis," Bull Physiopathol Respir, 11(2), 231-244, (1975).

[196] Yin, Y., Hoffman, E. A., Ding, K., Reinhardt, J. M., and Lin, C.-L., "A cubic B-spline-based hybrid registration of lung $\mathrm{CT}$ images for a dynamic airway geometric model with large deformation," Physics in Medicine and Biology, 56(1), 203-218, (2011).

[197] Yin, Y., Hoffman, E. A., and Lin, C.-L., "Local tissue-weight-based nonrigid registration of lung images with application to regional ventilation," SPIE Conf. Medical Imaging, 7262, 72620C, (2009).

[198] Yin, Y., Hoffman, E. A., and Lin, C.-L., "Mass preserving non-rigid registration of CT lung images using cubic B-spline," Medical Physics, 36(9), 4213-4222, (2009).

[199] Yuan, S., Frey, K., Gross, M., Hayman, J., Arenberg, D. et al., "Semiquantification and classification of local pulmonary function by $\mathrm{V} / \mathrm{Q}$ single photon emission computed tomography in patients with non-small cell lung cancer: potential indication for radiotherapy planning," Journal of thoracic oncology, 6(1), 71-78, (2011).

[200] Yushkevich, P. A., Piven, J., Hazlett, H. C., Smith, R. G., Ho, S., Gee, J. C., and Gerig, G., "Userguided 3D active contour segmentation of anatomical structures: Significantly improved efficiency and reliability," Neuroimage, 31(3), 1116-1128, (2006). 
[201] Zijdenbos, A. P., Dawant, B. M., Margolin, R. A., and Palmer, A. C., "Morphometric analysis of white matter lesions in MR images: method and validation," IEEE Transactions on Medical Imaging, 13(4), 716-724, (1994).

[202] Zitova, B., and Flusser, J., "image registration methods: a survey," Image and Vision Computing, 21, 977-1000, (2003). 


\section{LIST OF ABBREVIATIONS}

\begin{tabular}{|c|c|}
\hline$n-\mathrm{D}$ & $n$-dimensional, $n \in\{2,3,4\}$ \\
\hline 4-D CT & Four-dimensional computed tomography $(3-D+$ time $)$ \\
\hline $\mathcal{C}$ & Cost function \\
\hline $\mathrm{CT}$ & Computed tomography \\
\hline DIR & Deformable image registration \\
\hline $\mathbf{E}$ & Lagrangian strain tensor \\
\hline $\mathbf{F}$ & Deformation gradient tensor \\
\hline FFD & Free-form deformation \\
\hline $\mathbf{G}$ & Eulerian strain tensor \\
\hline$I_{R}(\xi)$ & Reference (fixed or target) image \\
\hline$I_{M}(\xi)$ & Moving (or object, or floating, or test, or deformable) image \\
\hline IR & Image registration \\
\hline $\mathbf{J}$ & Jacobian \\
\hline MI & Mutual information \\
\hline NMI & Normalized mutual information \\
\hline$\Omega \subset \mathbb{R}^{3}$ & Region of interest captured by the image \\
\hline$\rho(\xi, t)$ & Intensity function \\
\hline $\mathcal{R}$ & Regularization or Penalty term \\
\hline RT & Radiation therapy \\
\hline $\mathcal{S}$ & Similarity \\
\hline$T$ & Transformation \\
\hline $\boldsymbol{u}(\xi)=(u, v, w)$ & Displacement vector \\
\hline$v(\xi)=\frac{d u(\xi)}{d t}$ & Velocity vector \\
\hline$x\left(\xi, t_{1}\right)$ & Lagrange coordinate of the point $\xi$ \\
\hline$x\left(\xi, t_{2}\right)$ & Euler coordinate of the point $\xi$ \\
\hline
\end{tabular}




\section{CURRICULUM VITAE}

NAME:

ADDRESS:

EDUCATION:
Mohammadreza Negahdar

Lutz Hall, Room 308

Electrical and Computer Engineering Department

University of Louisville,

Louisville, KY 40292

Tehran, Iran - August 22, 1979

B.S., Biomedical Engineering (Bioelectric)

Science and Research College, Tehran, Iran

1999-2003

M.S., Biomedical Engineering (Bioelectric)

Tehran University of Medical Sciences, Tehran, Iran

2004-2006

Ph.D., Electrical and Computer Engineering

University of Louisville, KY, USA

2007-2012

HONORS \& AWARDS:

Chosen as the winner of Electrical Engineering Outstanding Graduate Student Award at the J.B. Speed School of Engineering Annual Honors and Awards Program, University of Louisville, A pril 2012.

Chosen as a finalist in the SPIE Medical Imaging Symposium Best Student Paper Award Competition (one of the 8 for the entire symposium) for the paper: "A 3-D Optical Flow Technique Based on Mass Conservation for Deformable Motion Estimation from 4-D CT Images of the Lung", Mohammadreza Negahdar and Amir A Amini, SPIE Medical Imaging Symposium, San Diego, CA, February 2012.

Chosen for the 2012 Who's Who Among Students in American Universities and Colleges in recognition of outstanding merit and accomplishment, representing the University of Louisville, January 2012.

Letter of commendation from Peter Rowell, Ph.D., Associate Dean for Research at University of Louisville School of Medicine on having one of the best posters (amongst 300) in Research!Louisville for a poster entitled, "Planar Deformable Motion Estimation Incorporating Mass Conservation and Image Gradient Constancy", October 2011. 
Ranked in the top 5 percent of graduate students at the University of LouisvilleGolden Key Honour Society, 2009.

Proclaimed as most productive researcher student in Research center for Science and Technology in Medicine (RcSTIM), 2006.

Young investigator fellowship, German Academic Exchange Program, 2006.

Ranked as top student in the Department of Biomedical systems and Medical

Physics, Tehran University of Medical Sciences, 2005.

First rank in nation-wide entry exam for the M.S. degree amongst 30,000 students, 2003.

\section{SERVICE:}

Journal Reviewer:

Conference Reviewer:

Program Committee:
IEEE Transactions on Medical lmaging.

Elsevier Pattern recognition.

International Conference on Medical Image Computing and Computer Assisted Intervention (MICCAI).

SPIE Conference on Biomedical Applications in Molecular Structural, and Functional Imaging.

International Symposium on Biomedical Imaging (ISBI).

International Conference of the IEEE Engineering in Medicine and Biology Society (EMBC).

MIICCAI Workshop on Interdisciplinary Clinical Software Support, 2012.

\section{PROFESSIONAL SOCIETIES:}

Institute of Electrical and Electronics Engineers (IEEE), Since 2007.

IEEE Engineering in Medicine and Biology Society (EMBS), Since 2007.

Technology, Entertainment, Design (TED), Since 2007.

Society of Photo-Optical Instrumentation Engineers (SPIE), Since 2009.

PATENT: $\quad$ Mohammadreza Negahdar, Amir Amini, Neal Dunlap, and Shiao Woo, "Volumetric Deformable Registration Method for Thoracic 4-D Computed Tomography Images," U.S. Provisional Patent, Application \# 61/618,081.

\section{PUBLICATIONS:}

1. Mohammadreza Negahdar, Neal Dunlap, Albert Zacarias, A Cahid Civelek, Shiao Y Woo, and Amir A Amini, "Strain as a Novel Index of Regional Pulmonary Function from Thoracic 4-D CT: In-Vivo Validation with Tomographic SPECT Ventilation and Perfusion, " submitted to Medical Physics. 
2. Mohammadreza Negahdar and Amir A Amini, "A Multi-Scale Optical Flow Technique Incorporating Mass Conservation for Deformable Motion Estimation (MOFID) from 4-D X-ray CT Images of the Lung," submitted to IEEE Transactions on Medical Imaging.

3. Mohammadreza Negahdar, Amir A Amini, Albert Zacarias, Bart L Lynch, Rebecca A Milam, Shiao Y Woo, and Neal Dunlap, "Strain as a Novel Index of Regional Function from Treatment Planning Thoracic 4-D CT, "Journal of Thoracic Oncology, supplement for the 2012 Chicago Multidisciplinary Symposium in Thoracic Oncology, Chicago, IL, USA, Sep 2012.

4. Mohammadreza Negahdar and Amir A Amini, "Regional Lung Strains via a Volumetric Mass Conserving Optical Flow Model, " IEEE Int. Symposium on Biomedical Imaging (ISBI), Barcelona, Spain, May 2012.

5. Mohammadreza Negahdar, Albert Zacarias, Rebecca A Milam, Neal Dunlap, Shiao Y Woo, and Amir A Amini, "An Automated Landmark-based Elastic registration Technique for large Deformation Recovery from 4-D CT Lung Images, " Proceedings of the SPIE Conference on Biomedical Applications in Molecular, Structural, and Functional Imaging, 8317-51, San Diego, CA, USA, Feb 2012.

6. Mohammadreza Negahdar and Amir A Amini, "A 3-D Optical Flow Technique Based on mass Conservation for Deformable Motion Estimation from 4-D CT Images of the Lung, " Proceedings of the SPIE Conference on Biomedical Applications in Molecular, Structural, and Functional Imaging, 8317-50, San Diego, CA, USA, Feb 2012 [Chosen as a finalist in Medical Imaging Best Student Paper Award competition].

7. Mohammadreza Negahdar, Hui Wang, and Amir A Amini, “ A Novel Optical Flow Method for Myocardial Deformation Analysis from Tagged MRI, "SCMR-ISMRM Workshop on Exploring New Dimensions of cardiovascular Flow and Motion, Orlando, FL, USA, Feb 2012.

8. Mohammadreza Negahdar and Amir A Amini, "Multi-scale Optical Flow Including Normalized Mutual Information for Planar Deformable Lung Motion Estimation from 4-D CT, " $33^{\text {rd }}$ IEEE EMBS Annual Int. Conf., 4888-4892, Boston, MA, USA, Sep 2011.

9. Mohammadreza Negahdar and Amir A Amini, "Estimation of Affine Motion from Projection Data Using a Mass Conservation Model, " $33^{\text {rd }}$ IEEE EMBS Annual Int. Conf., 8041-8044, Boston, MA, USA, Sep 2011.

10. Mohammadreza Negahdar and Amir A Amini, "Planar Deformable Motion Estimation Incorporating Mass Conservation and Image Gradient Constancy," IEEE Int. Symposium on Biomedical Imaging (ISBI), 536-540, Chicago, IL, USA, Mar 2011 [NIH travel grant award].

11. Mohammadreza Negahdar and Amir A Amini, "Tracking Planar Lung Motion in 4-D CT with Optical Flow: Validations and Comparison of Global, Local, and Local-Global Methods," Proceedings of the SPIE Conference on Biomedical Applications in Molecular, Structural, and Functional Imaging, 7626-74, San Diego, CA, USA, Feb 2010.

12. Mohamadreza Negahdar, Alireza Ahmadian, and Nassir Navab, "Stiff and Efficient Middle Axis Extraction of Airways for Virtual Bronchoscopy, " Int. Conference of Telemedicine and e-Health, Tehran, Iran, Nov 2006. 
13. Mohamadreza Negahdar, Alireza Ahmadian, Nassir Navab, and Kavous Firouznia, "Path Planning for Virtual Bronchoscopy," $28^{\text {th }}$ IEEE EMBS Annual Int. Conf., 156-159, New York, NY, USA, Sep 2006.

14. Mohamadreza Negahdar, Alireza Ahmadian, Nassir Navab, and Kavous Firouznia, "Automated Path Planning Algorithm for Virtual Bronchoscopy, " Iranian Journal of Medical Physics (IJMP), 3(11), 51-62 (2006). 